\title{
Index of Abstract Authors
}

Aamelfot, Christina..................... 1908 Aas, Hans ..................................... 110

Aas, Turid........................................ 110

Abbas, Jaber.................................... 178

Abbas, Jad ........................................ 959

Abbas, Ossama.............................. 1259

Abbott, Sara ................................. 1121

Abbuhl, Mary F........................... 2002

Abdel-Wahab, Omar ........................ 64

Abdul Rahman, Rozana ............... 1762

Abdul-Karim, Fadi W .................... 481

Abdul, Rehman .......... 101, 706, 1691

Abdulameer, Shahad ................... 2088

Abdulfatah, Eman ..... 102, 251, 1083 ,

1084,1217

Abdulrahman, Ahmed ...................... 103

Abe, Hiroyuki ............................................. 619

Abe, Sousei .................................. 1866

Abed, Francisco ........................................... 871

Abedalthagafi, Malak ................... 1696

Abel, Haley ........................................514

Abendroth, Catherine S.................. 451

Abi Raad, Rita.............. 124, 341, 342

Aboshady, Ibrahim ........................... 313

Abou-Ouf, Hatem ........................ 1062

Abraham, Ronald ........................ 1957

Abraham, Susan C ............... 623, 679

Abramson, Jeremy ....................... 1337

Abu-Farsakh, Sohaib....................... 620

Abudayyeh, Ala.............................. 912

AbuSaadeh, Feras ......................... 1191

Abusada, Ellen ............................. 2000

Abuzenadah, Adel ............................. 631

Achim, Mary ................................. 1061

Ackerman, Michael J ..................... 324

Acosta-Gonzalez, Gabriel......... 1629,

1654

Adam, Benjamin .............. 1585, 1627

Adem, Patricia V .............................. 69

Adeniran, Adebowale........... 341, 342, $353,841,915,952,1026$

Adeyi, Oyedele .

Adhikari Guragain, Deepti.......... 1213

Adlowitz, Diana ........................... 1398

Adsay, Volkan ..... 351, 392, 393, 636, $772,773,826,1666,1738,1740$,

$1748,1759,1763,1764,1765$,

1766, 1767, 1771, 1772, 1784

Adusumilli, P .............................. 1886

Adwar, Wamidh ........................... 1085

Ae, Keisuke....................................... 93

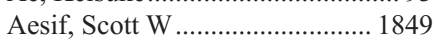

Affolter, Kajsa....................... 621, 716

Afify, Alaa .................................... 1588

Agaimy, Abbas ................ 1086, 1258

Agaram, Narasimhan P ........... 33, 58,

1836

Agarwal, Atin .........................622, 623

Agarwal, Jai P ............................... 1288

Agarwal, Nitin K.............. 1370, 1495

Agboola, Ayodeji O......................... 195

Aggarwal, Gitika............................. 198

Aggarwal, Nidhi..... 1415, 1436, 1463

Agostini-Vulaj, Diana ................... 624

Agoston, Agoston........ 625, 626, 700,

742,752

Agrawal, Tanupriya............ 831, 1630
Aguayo, Gloria................ 1738, 1772

Aguilera-Barrantes, Irene............ 1107

Aguiluz, Angela ............................ 1872

Aguirre, Mercedes........................... 421

Ahearn, Thomas .............................. 1065

Ahlborn, Lise ............................... 1269

Ahmad, Salman............................ 1384

Ahmadi, Parnian ................................ 61

Ahmed, Isthiaque .......................... 1575

Ahmed, Shahida ............................ 1342

Ahmed, Syed Salahuddin............... 104

Ahn, Hyein ........................................ 101

Ahn, Inhye.................................... 1507

Ahn, Janice........................ 1325, 1327

Ahn, Sangjeong............ 627, 720, 760

Ahn, Soomin ......................... 720, 796

Ahrendt, Gretchen.......................... 129

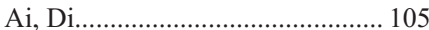

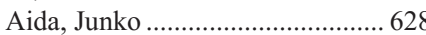

Aida, Noriko ................................ 1846

Ainechi, Sanaz ..... 629, 716, 717, 842

Ait si ali, Slimane......................... 1676

Aizer, Ayal A ................................. 1696

Akbani, Rehan.................................... 846

Akbari, Mohammad R ................ 1087

Akdas, Yasemin................................. 542

Akgul, Mahmut ............................... 843

Akgun, Hulya ......................................... 1

Akhter, Ariz............. 1361, 1417, 1469

Akhter, Shabnam......................... 1088

Akiba, Jun ...................................... 1223

Akiyama, Yoshiyuki...................... 844

Akkas, Gizem.......... 773, 1738, 1772

Akki, Ashwin ................................. 845

Akram, Muzaffar......... 198, 253, 268,

277

Akslen, Lars A..................... 110, 1908

Al Ahmadie, Hikmat .................... 1036

Al Diffalha, Sameer ....................... 749

Al Rawi, Sumaya ............................ 182

Al Zahrani, Ali Matar................... 1361

Al-Ahmadie, HA ............................. 910

Al-Ahmadie, Hikmat................... 1080

Al-Ahmadie, Hikmat A....... 846, 847, 939, 940, 1000, 1800

Al-Ahwal, Mahmoud ..................... 631

AL-Badri, Osamah........................... 1586

Al-Dandan, Sadeq ....................... 1724

Al-Delfi, Firas .............................. 1587

Al-Habib, Ali............................... 1327

Al-Haddad, Sahar......................... 1033

Al-Hilli, Zahraa............................... 119

Al-Ibraheemi, Alyaa............ 35, 36, 37

Al-Kawaaz, Mustafa .................... 1589

Al-Maghrabi, Jaudah...................... 631

Al-Niaimi, Ahmed......................... 1257

Al-Quran, Samer Z....................... 1187

Al-Rohil, Rami N................ 488, 515

Alaggio, Rita .............. 65, 1461, 1836

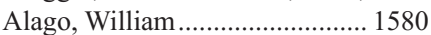

Alam, Elie ..................................... 1259

Alatassi, Houda ............ 475, 477, 911 ,

1498

Alayed, Khaled ................ 1326, 1456

Albacker, Lee ............................... 1785

Albadine, Roula …………………..... 343

Albanese, Joseph............................ 222
Albany, Costantine 941

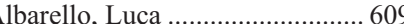
Albarracin, Constance .................... 147 Albero, Raquel ........ 960, 2024, 2045, 2055, 2077

Albert, Sebastien ............................ 1301 Albieri, Roberta AP...................... 2038

Albrecht, Hana ............................... 1631

Albuquerque, Miguel ...... 1672, 1676,

1677

Alcaraz-Mateos, Eduardo ............. 543

Aldecoa, Iban ........... 630, 1844, 2042

Alden, Ryan S ............................. 1922

Alderman, Megan ........................... 344

Alderuccio, Juan ................................. 1495

Aldrees, Sultan .............................. 1737

Aldulescu, Monica ........................ 1945

Alegre, Victor...................................527

Alejo, Maria .................................... 1194

Alemany, Laia ..................... 871, 1194

Aleskandarany, Mohammed A.... 195,

$196,242,243$

Alex, Deepu ...................................... 34

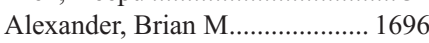

Alexander, Erik K ................. 599, 613

Alexander, Mariam P ........ 1586, 1951

Alexander, Mariam Priya ............ 1618

Alexis, Claudine............................. 2015

Alford, Sharon H.......................... 1089

Ali-Fehmi, Rouba ....... 102, 251, 273,

$398,1083,1084,1090,1217,1228$

Ali, Asma ........................... 558, 1432

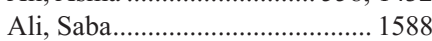

Ali, Shadan..........................639, 1825

Ali, Simak ……………................. 242

Ali, Siraj M ......... 146, 241, 271, 528,

$611,701,758,827,842,1025,1123$, $1174,1260,1287,1776,1785$, $1795,1853,1927$

Ali, Syed Z ........................... 370, 399

Ali, Waleed..................................... 848

Alikhan, Mir................................ 1328

Alizadeh, Layla ............................ 1187

Aljinovic, Nika.............................. 1615

Alkan, Serhan...................... 202, 1421

Alkapalan, Deema............................ 568

Alla, Ravi K ...................... 1792, 2052

Allaf, Mohamad E.............. 927, 1053

Allard, Felicia ............................... 1739

Allen, Brian.................................... 1214

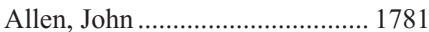

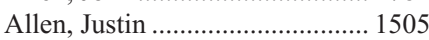

Allen, Peter .................................. 1740

Allende, Daniela .... 1662, 1663, 1664

Allison, Derek B ………………..... 345

Allison, Kimberly ...................114, 1973

Allmer, Cristine............................. 1363

Allo, Ghassan .................... 1085, 1089

Almadani, Noorah....................... 1153

Almeida de Jesus, Adriana .......... 1520

Almeida, Madson Q......................... 617

Almeida, Raquel .......................... 1193

Almenara, Gorge A ....................... 1990

Almenara, Jorge .................................. 471

Almutairi, Fawaz........................... 849

Alobeid, Bachir...... 1401, 1992, 1993

Alomari, Ahmed.................... 487, 537
Alonso, Sonia ................................ 527

Alos, Llucia......................................... 1289

Alosh, Baraa............. 251, 1083, 1090

Alpers, Charles............................. 1591

Alpert, Lindsay .......................... 632,792 ,

1632

Alruwaii, Fatimah ....................... 1083

Alsaati, Gazal................................. 346

Alsafadi, Muhammad K...... 102, 251, 890,1083

AlSaikhan, Bader ............................ 901

AlSalihi, Suhair A ….......................... 1633

Alsomali, Mohammed.................... 634

Altree-Tacha, David........................ 499

Alturkustani, Murad....................... 2025

Alva, Ajjai ...................................... 988

Alvarado-Cabrero, Isabel.... 287, 850,

1027

Alvarado, Anthony S................... 1591

Alvarez Secord, Angeles ............. 1121

Aly, F Zahra ..........................348, 1110

Amador-Ortiz, Catalina............... 1365

Amador, Catalina .............. 1352, 1366

Ambaye, Abiy B............................. 294

Ambelil, Manju ............................. 1633

Ambrosone, Christine ...................... 193

Ameri, Maryam D ...................... 1329

Amin, Ali........... 928, 977, 979, 1230

Amin, Khalid..................... 452, 1988

Amin, Mahul B ........ 933, 986, 1008, 1012, 1030, 1045, 1047, 1048

Amin, Shahrier.................... 314, 1590

Amin, Mitual....................... 1368, 1457

Amorese, Rebecca.................624, 640

Amos, Christopher I......... 1787, 1928

An, Soyeon............. 1750, 1753, 1754

Anand, Mona.......

1170

Ananthanarayanan,

Vijayalakshmi ........... 1850, 1923,

1945

Anastasi, John .......... 558, 1434, 1504

Andea, Aleodor ............. 503, 515, 537

Andea, Aleodor A........................... 487

Andeen, Nicole .......... 851, 855, 1591

Anders, Robert ............................... 806

Anders, Robert A............................. 703

Anderson, Blake...................... 852, 882

Anderson, Carol ........................... 450

Anderson, Courtney ......................... 2026

Anderson, Glenda ......................... 1549

Anderson, James ………………….... 85

Anderson, Matthew L ....................... 1167

Anderson, Neil H ......................... 1777

Anderson, Virginia ............................ 19

Andrada, Encarna................................... 1197

Andrasova, Martina......................... 543

Andre, Fabrice............................... 1822

Andrews, Caitlin ............................ 106

Andrews, David ............................... 824, 1825

Andrulis, Irene ............................. 1967

Aneja, Ritu ........... 195, 196, 200, 207 ,

238, 242, 243, 244, 304, 826, 2059

Angchuan, Siripron ...................... 1592

Angelini, Annalisa........................... 329

Angell, Trevor ....................... 599, 613

Angelos, Peter ................................... 575

Angert, David................................... 315 
Angevin, Eric ................................ 1822

Angot, Emilie................................... 38

Anichini, Andrea ............................ 592

Anita, Kumar.................................... 1481

Annan, Anand ............................... 1946

Ansari, Junaid ....................... 92, 1819

Ansell, Wendy ............................. 1038

Antic, Tatjana ...... 368, 575, 582, 851,

855

Antonarakis, Emmanuel S ... 931, 984

Antonescu, Cristina..... 33, 47, 58, 65, $1294,1304,1316,1836$

Aoyama, Chisa ................................ 1241

Aplenc, Richard ......................... 1515

Aplin, Alfred C................................ 316

Appiah, Adams K......................... 1352

Appleman, Leonard J .................. 1017

Aprikian, Armen .............................. 901

Aqil, Barina.................................. 1330

Arabe, Jorge A................................. 421

Arafah, Maria .................................. 137

Arai, Tomio ............................ 333, 628

Araki, Asuka .................................. 735

Arana, Inigo ................................. 1706

Aranake-Chrisinger, John ............ 1091

Aranda, Derick M ........................ 1724

Araujo, Karina S .................. 866, 867

Araya, Juan Carlos .......... 1738, 1763,

1764,1772

Arber, Daniel................................. 1350

Arber, Daniel A ..... 1331, 1364, 1392, $1427,1430,1486,1508$

Arcaro, Kathleen F...................... 1802

Arcila, M..................................... 1894

Arcila, Maria E...... 57, 64, 847, 1375,

$1382,1803,2075$

Ardeniz, Omur .................................. 766

Areas, Ana Lucia G..................... 2038

Arend, Lois …………………........ 1602

Argani, Pedram ..... 65, 239, 293, 980,

1028

Arguelles-Grande, Carolina ....... 1401

Argyris, Prokopios ....................... 1261

Arias-Stella, Javier...... 107, 186, 853,

1040

Arif, Qudsia................................... 1850

Arita, Junichi..................... 1660, 1661

Ariza, Aurelio.................................. 746

Arkin, Jordan.................................. 347

Arksarapuk, Jittirat....................... 1592

Armesto, Susana …........................... 527

Armitage, James........................... 1454

Armylagos, Donna .............. 352, 1970

Arnald, David J ............................ 1312

Arnason, Thomas ............................. 732

Arnold, Christina A..... 634, 686, 788,

1832

Arnold, Michael ................. 634, 1832

Arnold, Shanna .............................. 892

Arnold, Stacy J......................348, 1110

Arnould, Laurent............................ 254

Aronson, Judith .................................. 21

Arora, Arshi................................... 1910

Arora, Kshitij ..................... 821, 1135

Arps, David P................................. 531

Arriola, Aileen Grace P.............. 1262

Arrossi, Andrea V ............ 1889, 1895

Arshad, Muhammad........................ 204

Arun, Banu ..................................... 147

Asano, Naoko................................. 1359

Asato, Marcel A .................... 866, 867
Asatrian, Greg ................................... 79

Asch-Kendrick, Rebecca J ............ 293

Asgedom, Girmay ................ 187, 235

Ash, Ryan....................................... 1631

Ashok, Dhandapani........................ 757

Asioli, Sofia...........................569, 618

Asirvatham, Jaya R ....................... 108

Askan, Gokce............................... 1740

Askin, Frederic.............................. 1875

Aslanian, Harry …………………..... 813

Asleh-Aburaya, Karama ................ 281

Assaad, Adel ...................... 635, 1316

Assaad, Adel M............................. 1271

Assarzadegan, Naziheh ........ 489, 675

Assis, David .................................. 1659

Aster, Jon C.................................. 1462

Astrow, Stephanie H ................... 1092

Astvatsaturyan, Kristine............. 109, 309

Aswad, Bassam ................................. 415

Ataya, Dana...................................... 310

Atieh, Mohammed .... 838, 839, 1790

1991

Atkins, Kristen A................. 162, 1181

Attwooll, Claire.......... 71, 1409, 2031

Aubry, Marie Christine ......... 4, 1893

1911

Aubry, Marie-Christine....... 15, 1849 ,

1884

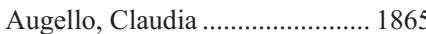

Augello, Michael............................ 1816

Auger, Manon ........................ 362, 397

Auger, Nathalie .............................. 1822

Auguste, Louis J................. 165, 1563

Augustin, Jeremy ........................... 1263

Aull, Meredith J ........................... 1619

Auman, Heidi .................................. 993

Aung, Phyu P ...... 488, 490, 523, 530,

535,540

Austin, Marshall R .......................... 462

Austin, Melissa ................................ 553

Austin, R Marshall ......................... 479

Avadhani, Vaidehi ....... 349, 350, 351, $392,393,580,636,1771,1964$

Avery, Diane..................................... 380

Aviki, Emeline M......................... 1144

Avila-Casado, Carmen ........................ 3

Avril, Stefanie ................................. 206

Avula, Rajeswari ............................. 324

Awad, Mark..................................... 1887

Awadallah, Amad ........ 379, 391, 661,

1326

Axiotis, Constantine...................... 704

Aya, Francisco............................. 1907

Ayala, Alberto G ............................. 997

Ayata, Gamze ................................... 1739

Aye, Le ........................................ 1092

Aykutlu, Umut.............................. 1692

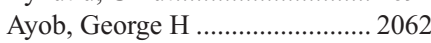

Ayre, Gareth ................................. 1282

Azad, Nilofer S ................................. 812

Azadmanesh Samimi, Siavash ..... 352

Azaro, Analia ................................. 1813

Aziz, Mohamed S............................ 360

Aziz, Sara .................................... 1002

Aziz, Sura M .................................. 110

Aziz, Tariq...................................... 361

Azueta, Ainara.................................... 677

Azzato, Elizabeth M...................... 1796

Baba, Satoshi................................. 532

Babayeva, Sabina..... 854, 1851, 1852

Babushok, Daria........................... 1486
Bacchi, Carlos E........111, 1279, 1294 Badoual, Cecile ...... 1263, 1301, 1834 Badve, Sunil ......................... 112, 161

Bae, Jeong Mo ................................ 753

Bae, Young K ................................ 1742

Baehner, Frederick L....................... 113

Baek, Seunghee.............................. 459

Baergen, Rebecca......................... 1838

Baetz, Tara .................................... 1417

Bagci, Pelin ............. 772, 1763, 1765

Bagg, Adam............ 1473, 1486, 1508

Baggerly, Keith .............................. 891

Bagnasco, Serena ........................... 1602

Bagrodia, Aditya ................ 847, 1000

Bahar, Burak ......................... 384, 855

Bahary, Nathan............................ 1776

Bahrami, Armita................................. 39

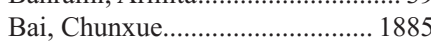

Bai, Yalai ...................................... 1109

Bailey, Helen.................................... 113

Bailey, Mark.................................. 528

Bailey, Nathanael G .................... 1439

Baine, Marina................................... 125

Bains, S ..................................... 1886

Baiocchi, Robert .............................. 1335

Bajaj, Renu................................... 2027

Bajorin, Dean F.......... 847, 939, 1000

Baker, Gabrielle M................ 220, 221

Baker, Suzanne J ......................... 1735

Bakhiet, Salih ............................. 1251

Bakshi, Ganesh ........................... 995

Bal, Munita .... 796, 1288, 1297, 1300

Balabaud, Charles ....................... 1638

Balabram, Debora ……………….... 711

Balague, Olga.............................. 1483

Balaguer, Francesc .......................... 630

Balakrishna, Jayalakshmi P.......... 734

Balakrishnan, Dhanalakshmi ..... 1947

Balassanian, Ronald..... 121, 122, 431

Balasubramanian, Ishwaria ........... 680

Balatti, Veronica............................. 789

Balci, Serdar......... 636, 772, 773, 826

Baldassarri, Rebecca.... 341, 342, 353

Baldridge, Lee Ann ....................... 873

Balitzer, Dana............................... 1634

Balk, Steven ................................. 1078

Ball, Mark W..................... 883, 1053

Ballard, Morgan .............................. 114

Ballo, Michael S.............................. 447

Ballon, Gianna ..................... 360, 491

Baloch, Zubair.......................... 564, 605

Balogh, Zsofia .............................. 1822

Baltatzis, George ............................. 1497

Baltay, Michele ………………..... 1462

Baltazar, Fatima …….......................... 617

Balzer, Bonnie L .......................... 1047

Bander, Neil H ............................. 1270

Bandy, Andrew...................1532, 1635

Bandyopadhyay, Sudeshna ......... 102,

251, 273, 398, 1083, 1084, 1090, $1217,1228,1763,1765$

Bangs, Dana ................................. 1431

Banito, Ana ........................................ 50

Bannan, Michael ............................ 616

Baqai, Saad ....................................... 1264

Baras, Alexander ......... 448, 511, 563, $856,927,961,963,1976$

Barber, Jason K... 1709

Barberis, Massimo ……………....... 1865

Barbieri, Andrea L............... 341, 1948

Barbieri, Christopher E ............... 1002
Barboza, Oralia ........................... 1130

Bareja, Rohan................... 1024, 1786

Barkan, Guliz ................................ 885

Barkan, Guliz A... 362, 384, 438, 542,

Barkoh, Bedia A..... 444, 1957, 2003

2058

Barletta, Justine A ....... 458, 599, 613,

Barnhill, Raymond ........................ 505

Baro, Teresa ...................................... 2077

Barod, Ravi ............. 917, 1074, 1076

Barr Fritcher, Emily G ............... 1724

Barraza, Gonzalo................. 115, 1949

Barrese, Tomas Z …..................... 1418

Barreto, Jose.................................. 870

Barrett, Amanda .............................. 557

Barrett, Mary .......................... 857, 858

Barrett, Megan R........................ 1158

Barroca, Helena.............................. 556

Barroeta, Julieta E ....................... 1222

Barrows, Brad ..................... 1593, 1626

Barry Delongchamps, Nicolas ... 2090

Barry-Holson, Keegan ................. 1093

Barry-O'Crowley, Jacqueline .... 1317

Barry-O'Crowley, Jacqui .......... 1099,

1243

Barry, Beatrix ................................ 1301

Bartels, Ashley ............................. 1384

Bartlett, Jamen ............................. 1094

Bartosch, Carla.................... 258, 1203

Barua, Animesh............................ 1095

Basavanhally, Ajay ....................... 1561

Basbug, Mustafa .................................. 1

Baselga, Jose ....................... 268, 270

Basir, Zainab ................................. 170

Baskin, Leland B........................... 338

Baskovich, Brett.............................. 555

Basnet, Kristen M ............................... 1096

Basolo, Fulvio ....................... 570, 571

Bassano, Cristina............................ 264

Bassat, Quique ........................... 1535

Bassett, Roland L ........ 150, 307, 371,

$535,1124,1125$

Bassiouny, Dina ..... 1087, 1097, 1098

Basso, Cristina ...... 328, 329, 330, 332

Bast, Martin................................ 1454

Bastacky, Sheldon I... 354, 859, 1017 ,

Bastard, Christian 1018

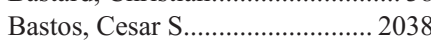

Bastos, Lucas QA.................. 866, 867

Basturk, Olca... 772, 773, 1738, 1740 ,

1765,1772

Basu, Sankha S............................. 1332

Batalis, Nicholas ................... 550, 551

Batdorf, Bjorn ................................ 1333

Bateman, Justin ............. 860, 861, 862

Bates, Mark ................................. 1099

Batlevi, Connie ................. 1382, 1803

Batlle, Ana..................................... 1418

Batra, Surinder K .......................... 1322

Battaglia-Hsu, Shyue-Fang ........ 1698

Baudhuin, Linnea M ...................... 324

Baumgartner, Erin ............................ 921

Bavi, Prashant ..... 2, 3, 317, 637, 833,

834,1826

Baxter, Aaron J........................... 1428

Baydar, Dilek ............. 886, 887, 1004

Bayerl, Michael G........................ 1472

Baylin, Stephen 
Beach, Jordan R 1800 Beanlands, Robert ....................... 2025 Beattie, Adam............................ 1636 Beaubier, Nike ......... 424, 701, 1652, $1758,1807,1853$

Beca, Francisco ....................116, 117

Beck, Andrew H.........116, 117, 1938

Becker, Kent............................... 2000

Beckmann, Matthias W.............. 1086

Bedolla, Gabriela .... 826, 1738, 1765 ,

$1766,1767,1772$

Bedossa, Pierre....... 1672, 1676, 1677

Bedrosian, Isabelle....................... 147

Bedrossian, Kristi....................... 1996

Beg, Muhammad S........................ 653

Beggan, Caitlin ......................... 1265

Begnami, Maria........................... 1641

Begnami, Maria O....................... 1641

Behdad, Amir ................. 1334, 1467

Behm, Frederick........................... 793

Behrens, Carmen......................... 2081

Bejarano, Pablo A.......................... 638

Belanger, Eric C... 860, 861, 862, 950

Belchis, Deborah....................... 1854

Bell, Debra A.............................. 1254

Bell, Diana ..... 534, 1266, 1314, 1705

Bell, Douglas.................................. 480

Bell, Sarah.................................. 1100

Bellara, Aarti ................................ 558

Bellevicine, Claudio............. 465, 808

Bellizzi, Andrew M............ 496, 526, 568, 581, 798, 1381, 1745, 1778,

1950,2000

Bellmunt, Joaquim ...................... 846

Bellocq, Jean-Pierre ............ 135, 254

Bellolio, Enrique R ........ 1738, 1763,

1764, 1772

Bellur, Shuba S ......................... 1622

Beltran, Himisha ............ 23, 440, 889, 1024, 1786, 1816, 1828, 2035

Beltran, Luis.................................. 863

Ben Dor, David ..............................111

Benayed, Ryma .............................. 57

Bender, Ryan P.......................... 1797

Benelli, Matteo................................ 889

Benini, Stefania................................ 86

Bennasar, Mar ............................ 1837

Bennett, Ana E ............................. 786

Bennett, Jennifer A.................... 1228

Bennett, John P .......................... 868

Bennett, Michael W .... 118, 126, 127, $128,733,1574$

Bennis, Malika ............................. 593

Bentley-Lewis, Rhonda.............. 1096

Bentley, James.............................. 1041

Berchuck, Andrew...................... 1121

Berczy, Margaret........................ 1840

Berezowska, Sabina ..................... 670

Berg, Aaron N .............................. 355

Berger, M .................................. 1894

Berger, Michael................ 795, 1000

Berger, Michael F......... 57, 270, 363, 418, 847, 939, 1080, 1080, 1814,

1827,1910

Berger, Mitchel S ....................... 1725

Bergholtz, Sarah............................. 91

Bergman, Jacques JGHM...... 672, 811

Bergsland, Emily K................... 1761

Berho, Mariana ............................ 638

Berjawi, Ghina ............................ 178

Berkowitz, Aaron L.................... 1720
Bernard, Philip ................... 164, 281

Bernardo, M Magarida................. 903 Berney, Daniel......... 863, 1038, 1062

Bernie, Aaron .............................. 1998

Beroukhim, Rameen ................. 1696

Berrebi, Alexander A.................. 1902

Berretta, Remus........................... 315

Berry, Gerald J ............................... 730

Berry, Ryan ............................ 40, 41

Bertaglia, Emanuele..................... 330

Berthelet, Eric .......................... 1282

Bertsch, Elizabeth ............... 864, 865

Bertuch, Alison A....................... 1399

Berzin, Tyler................................. 692

Beshai, Boulos ............................. 356

Best Rocha, Alejandro ............... 1594

Betz, Bryan ..................... 467, 1313

Beydoun, Rafic................ 1090, 1825

Beyene, Joseph............................ 361

Bezerra, Stephania ............ 886, 1004

Bezerra, Stephania M......... 883, 906, 934, 1053

Bhagat, Govind ...... 712, 1401, 1522 , 1992,1993

Bhalla, Amarpreet ..... 398, 639, 1637 Bhalla, Ritu ......................... 977, 979

Bhargava, Rohit .......... 166, 278, 279, $288,996,1238,2010$

Bhattacharyya, Siddharth............. 318

Bhattarai, Shristi ................. 207, 238

Bhatti, Rahat ............................. 1335

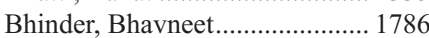

Bhuiya, Tawfiqul..... 165, 1563, 2015

Bi, Rui ............................1101, 1112

Bi, Wenya L............................... 1696

Bialik, Ann .................................. 1036

Bianchini, Diletta ....................... 1001

Bibars, Wafi................................ 1675

Bibbey, Scott ................................ 357

Bibeau, Frederic ........................... 671

Biemer, John ................................ 1533

Biernacka, Anna................ 358, 1787

Biernat, Wojciech........................ 1862

Bilir, Birdal ................................ 1811

Bilkey, Jade ............................... 1774

Billah, Shahreen ......................... 1465

Billings, Steven D .... 66, 67, 68, 506,

507,515

Billis, Athanase ................. 866, 867

Bilodeau, Elizabeth ...................... 1261

Binder, Scott.................................. 1267

Binot, Elke ................................. 1918

Binotti, Will............................... 1736

Bioulac-Sage, Paulette ............... 1638

Birdsong, George ........................ 1964

Birney, Laura................................ 1947

Birsa, Joe.................................... 1557

Bisagni, Alessandra...................... 264

Biscotti, Charles V .................... 1256

Bishop, John W .................... 121, 586

Bishop, Julie Anne .............911, 1102

Bishop, Justin A ...... 934, 1054, 1267,

$1274,1304,1305$

Bismar, Tarek ............................. 1062

Bisogna, Maria ............................. 1236

Biswas, Asok................................ 502

Bitterman, Pincas ......................... 1095

Bitting, Angela ............................ 1960

Bivalacqua, Trinity J ........... 856, 883

Bixby, Dale .............................. 1438

Bizzarro, Tommaso ........................ 468
Bjornhagen, Viveca.

Black, Candice C

Black, Jonathan ....

Blackler, Adele...

Blanchard, France

Blanco, Luis Z

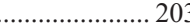

Blando, Jorge ............................. 750

Blanes, Alfredo

.. 897, 1794

Blank, Annika ............................... 585

Bledsoe, Jacob................. 1336, 1337

Bleiweiss, Ira J ..... 179, 180, 237, 267

Blessing, Melissa M.............. 4, 1951

Blevins, Lewis S ........................ 1715

Blosser, Christopher................... 1591

Boag, Alexander......................... 1939

Bobr, Aleh ................................. 1338

Boccardo, Simona ..................... 1861

Bochner, Bernard H .... 418, 847, 939,

Bockelmann, Robin................. 1779

Bocklage, Therese.... 40, 41, 455, 562

Bodalo, Marta ............................ 1289

Boddicker, Rebecca L ................. 1363

Bodo, Juraj ...................... 1445, 1480

Boehm, Steffen........................... 1229

Boerner, Scott............................... 1863

Boffa, Daniel................................. 813

Bogach, Jessica ............................... 361

Boger, Ravit ............................... 1703

Boguniewicz, Ann B ... 146, 241, 271,

Bohers, Elodie........................... 1809

Boi, Giovanna ............................. 2066

Boilesen, Eugene.......................... 1454

Bois, Melanie C ................. 119, 319

Boisselier, Blandine ................... 1727

Bolan, Charles................. 1357, 1405

Boland, Jennifer M..... 39, 1855, 1893

Boldt, Brandon ............................... 581

Bolster, Margot ................................ 18

Bolton, James .............................. 1086

Bolton, Warren K ....................... 1614

Boman, Bruce M........................... 676

Bomeisl, Philip.............................. 153

Bonanni, Luigi .......................... 1762

Bonazza, Deborah ................ 497, 498

Bond, Jesse................................. 1830

Bondaruk, Jolanta ............... 891, 935

Bondurant, Charles P ............... 1724

Bonert, Michael.................... 545, 546

Bongiovanni, Massimo ................ 468

Bonham, Michael J ............. 868, 989

Bonneau, Peter N ...... 396, 572, 1103 ,

Bonsib, Stephen M................... 957

Bonzheim, Irina........................... 1483

Bookhout, Christine ..................... 120

Boonipat, Thanapoom .................... 358

Boorjian, Stephen A..................... 999

Booth, Christine N ................ 240, 377

Borbolla, Mauricio........................ 543

Borczuk, Alain ............................ 780

Bordignon, Patrizia .................... 1302

Bordoni, Rodolfo ........................ 1260

Borges, Melissa ................................ 648

Boriack, Richard ....................... 1348

Borowitz, Michael J ................... 1429

Borowsky, Alexander... 121, 122, 586

Borsu, Laetitia .......................... 132

Borys, Adrianna ......................... 1576

Borys, Dariusz............................ 1576
Borys, Ewa................................. 1576

Bosari, Silvano ............................. 1865

Bosch, Francesc Xavier ............... 871

Bosch, Xavier............................. 1194

Bose, Shikha ...... 109, 123, 302, 309, 359,573

Bosenberg, Marcus W ................ 2074

Bosisio, Francesca.......................... 492

Boto, Agedi ......................... 124, 125

Boughey, Judy C .......... 229, 230, 305

Bougrini, Mouna ........................... 671

Boulianne, Patrice ....................... 2071

Boulos, Fouad ............................ 178

Bouvier, Nancy .......... 363, 418, 1827

Bowers, Kelly A......................... 2028

Bowman, Sarah............... 1626, 2033

Bowser, Zenica L ........ 201, 252, 253 ,

277

Boxerman, Jerrold L .................. 1728

Boyanton, Bobby ....................... 1605

Boyer, Hilary................................. 993

Boyer, James L............................ 1659

Boyer, Michael.................. 1293, 1308

Boyle, Sean M................ 1792, 2052

Boynton, Kathleen ........................ 621

Boysen, Gunther ............. 1001, 1002

Bozkurtlar, Emine ....................... 1920

Bracamonte, Erika..................... 1969

Bradley, Laura ............................... 641

Braga, Ana C.................. 1193, 1952

Brahmbhat, Rushin D.................. 204

Brand, Randall E........................ 1775

Brandler, Tamar C ........................ 360

Brandwein, Margaret ................... 1280

Branson, Julie................... 357, 1914

Bratton, Laura ................................ 640

Braxton, David R ......................... 1639

Braylan, Raul C............... 1356, 1357

Braziel, Rita ............................... 1449

Bream, Matthew........................... 843

Breau, Rodney H.................. 862, 950

Breese, Virginia............................. 928

Breining, Dwayne ......................... 924

Brenca, Monica ............................... 84

Brenin, Christiana M.................... 162

Brennan, Sandra .................... 252, 277

Breton, Quentin.......................... 1727

Brett, Mary Anne .......................... 361

Brewer, Molly ........................... 1252

Brewster, Ryan ............................ 1696

Briggs, Debra ................................. 1433

Brimo, Fadi ...... 362, 397, 901, 1033,

1070

Brinkerhoff, Brian T............... 5, 641

Bristow, Rob G................ 1019, 1067

Britton, Heidi ............................... 1242

Britton, Sarah ............................ 1605

Broach, James ................................ 1246

Broaddus, Emily ...... 642, 1104, 1788

Broaddus, Russell ....... 534, 642, 721,

1104, 1161, 1164, 1214, 1705, 1788

1957,2003

Broadwater, Gloria.................... 1121

Brochin, Lydia........................... 1698

Brodsky, Alexander.... 246, 758, 1123

Brodsky, Alexander S.......... 678, 823

Brodsky, Sergey ................ 1591, 1620

Brody, Judith ............................. 1380

Brody, Rachel............................... 185

Broehm, Cory ................................ 42 
Brogi, Edi.... 201, 252, 253, 259, 268, $270,277,1823$

Bronner, Mary P.................. 716, 728

Brooks, James D ................ 993, 1060

Brosnan-Cashman, Jacqueline...

Brown, Alan F ............................. 1339

Brown, David............................... 1731

Brown, Ian.................... 740, 741, 796

Brown, Krystal............................. 505

Brown, Larry................................ 1739

Brown, Noah ................... 1268, 1313

Brown, Robert.................. 1534, 1633

Brown, Ronald ............................. 1462

Brown, Ryanne............................... 547

Brown, Shari ............................... 1491

Browne, Tara Jane....... 118, 126, 127,

128,1574

Bruegl, Amanda .......................... 1214

Brufsky, Adam ...................... 129, 166

Brugarolas, James ............... 913, 914

Bruggeman, Richard D .............. 1246

Brunelli, Matteo .................... 873, 968

Brynes, Russell K......................... 1558

Bryson, Gareth W ....................... 1548

Bshara, Wiam ................................... 193

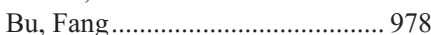

Buckingham, Lela ......................... 594

Buckland, Michael ...................... 1721

Budd, G T............................ 151, 152

Buehler, Darya .............. 90, 607, 618

Buelow, Benjamin............... 869, 1953

Bueno, Raphael ...............................625

Bueso-Ramos, Carlos E ............ 1422,

1464, 1473, 1486, 1508, 1509, 1526

Buettner, Reinhard ....................... 1918

Buhmeida, Abdelbaset .................... 631

Buhrer, Elias............................... 1482

Buhtoiarova, Tatiana .................... 1255

Bui, Marilyn ................................ 1328

Buiga, Rares................................... 287

Buja, L Maximilian ........................... 313

Bukelo, Maria F ............................. 1831

Bulimbasic, Stela ......................... 1010

Bull, Shelley................................. 1967

Bullock, Grant C .............. 1402, 1463

Bullock, Nyasha................... 198, 778

Bulman, William A......................... 460

Bulusu, Gautam.............................. 129

Buonocore, Darren ............. 363, 1954

Burack, Richard ..... 1397, 1398, 1452

Burdette, Elliott.................. 435, 2029

Burger, Peter ................................ 1703

Burke, Allen P............... 31, 32, 1902

Burke, Kathleen ........ 250, 268, 1250 ,

1798,1823

Burke, Louise................................. 18

Burmeister, Lynn............................. 452

Burnes, Catherine L ..................... 1415

Burnett, Alexander ....................... 2065

Burnett, Arthur ............................... 883

Burnier, Miguel N ............. 1736, 1737

Burns, Kathleen H......................... 1429

Burstein, David E......................... 1051

Burt, Michael M............................. 364

Burton, Emer.................................... 658

Busam, Klaus .................................. 505

Busby-Venner, Helene ................. 1698

Busca, Aurelia ............................... 1105

Busico, Adele ................................. 1903

Bussolati, Gianni............................ 556
Bustamante, Carlos D .................. 1807

Bustamante, Daniel .......................... 1340

Butany, Jagdish ..................... 2, 3, 317

Butko, Emerald ................................. 2026

Butnor, Kelly J .............................. 1094

Butz, Henriett....................... 896, 981

Buyukbabani, Nesimi................... 1307

Buza, Natalia............................... 1232

Buzelin, Marcelo A ……………........ 711

Buzzoni, Roberto ………………..... 592

Bydder, Graeme …....................... 2078

Byeon, Sun-ju ………………...... 1650

Byers, Richard................................ 2056

Byrnes, Kathleen.................. 574, 1640

Byrum, Stephanie........................ 1142

Caan, Bette J .................................... 164

Caballero, Fuensanta..........................543

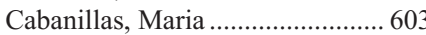

Cabecadas, Jose M ........................ 1483

Cacheux, Valere ………………..... 1273

Cadeddu, Jeffrey ............................ 913

Caglar, Derin .......................... 362, 397

Cahill, Dolores J............................. 1190

Cai, Changmeng............................ 1078

Cai, Donghong ............................... 1342

Cai, Guoping ......................... 341, 353

Cai, Shirong ..................................................... 691

Cai, Tommaso …………………... 1003

Cai, Xu ............................................. 1101

Cain, James …………………….... 1614

Caldas, Margarida ........................... 258

Calderone, Tiffany L ...................... 2064

Caldwell, Trevor ........................... 872

Calhoun, Benjamin C........... 151, 152,

$240,310,377$

Calio', Anna ......... 873, 874, 875, 968

Calkins, Sarah ............................... 365

Call, Michelle............................... 1549

Calonje, Eduardo...........................................

Calvo, Katherine R........... 1357, 1405,

1520

Camacho, Jessica ......................... 2073

Camacho, Jowaly ............................ 13

Cambor, Carolyn .................................. 548

Cameron, Stuart .............................. 182

Camilleri, Michael .......................... 824

Campbell, Frank.......................... 1234

Campbell, Natalie S ..................... 2005

Campiotti, Leonardo ..................... 1500

Campo, Elias ................................... 1483

Campos, Antonio.............................. 1641

Campuzano-Zuluaga,

$$
\text { German....... }
$$

1425,1550

Canales, Jaime Rodriguez............. 750

Canary Prostate Investigators .... 1060

Canepa, Mariana …………........... 415

Canete, Sofia ...... 870, 871, 909, 1035

Cani, Andi K ..................... 1278, 1805

Cannatella, John........................... 1953

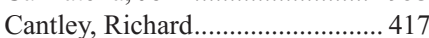

Cantuaria, Guilherme........... 195, 304,

2059

Cao, Dengfeng .............................. 876

Cao, Kai ...................................... 212

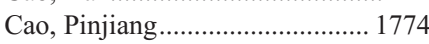

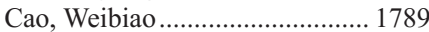

Cao, Wenqing ....................... 643, 1642

Cao, Xuhong .................... 1065, 1066

CAP Immunohistochemistry

Committee.................... 294, 1950

Capella, Carlo R............................... 609
Caraway, Nancy …………………... 425

Carballal, Sabela ............................. 630

Carballo-Zarate, Adrian ............... 1341

Carballo-Zarate, Adrian

Alejandro...

1457

Cardenas, Ana Maria................... 1537

Cardiff, Robert .............................. 121

Cardona, Diana M................. 668, 688

Cardoso, Manuel ............................ 1193

Carey, Kyle .....................................520

Carey, Thomas E ........................... 1268

Carlson, Erin E................................. 305

Carlson, Joseph .............................. 1106

Carneal, Eugene ............................ 1966

Carneiro, Fatima ………………...... 664

Caron, Alissa A.............................. 1725

Caron, Bolette L.............................. 163

Caron, Justin ....................... 130, 1955

Carpenter, Philip M........................ 121

Carrilho, Carla............................... 1535

Carrillo, Luis F.......... 644, 689, 1103 ,

1107

Carrio, Meritxell

... 83

Carroll, Martin .................. 1514, 1515

Carroll, Peter R ................................ 993

Carslon, J Andrew ........................... 528

Carstens, Billie J ……………….... 1485

Carter-Monroe, Naima ................. 1602

Carter, Cody ....................... 131, 1108

Carter, Gloria ................................... 129

Carter, Gloria J .............................. 2010

Carter, H Ballentine ............................. 970

Carter, Jamal H .................. 366, 1697

Carter, Jodi M ......... 35, 43, 119, 132,

Carter, John B

133

Carturan, Elisa.................................... 329, 330

Caruso, Anabela C ...................... 2038

Carvajal-Hausdorf, Daniel ........... 649,

Carvalho, Elizabete _. 11193

Carvalho, Filipe LF ....................... 812

Carvalho, Kelson R ............... 866, 867

Casanova, Jacklyn.............................. 64

Casanova, Ruben............................ 1938

Cascio, Michael J .......................... 1833

Cascione, Luciano................. 789, 790

Cason, Marco …………….... 329, 330

Casse, Jean-Matthieu ................... 1698

Cassivi, Stephen D ...................... 1911

Castain, Claire ................................ 1638

Castano, Ekaterina ........................ 1598

Castellano, Joan J........................... 1907

Castellanos, Elisabeth ....................... 83

Castells, Antoni .................. 630, 2042

Castellsague, Joan ............................... 49

Castillo-Martin, Mireia ...... 877, 1551

Castillo, Paola ....... 1220, 1535, 1577,

1837

Castonguay, Mathieu........... 732, 1921

Castro, Diana................................. 1342

Cataldi, Palmina .......................... 1294

Catchpoole, Daniel....................... 1515

Cates, Justin M....... 80, 674, 682, 945

Cathro, Helen ................................. 1614

Cattoretti, Giorgio ……………....... 2066

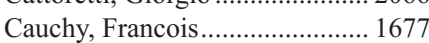

Cavallo, Allison B ………………....575

Cecchi, Fabiola ........................ 1813

Cecchini, Lluis ...................... 893, 960

Celeghin, Rudy ..................... 329, 330
Celli, Romulo .........................761, 813

Cenaj, Odise .......................... 645, 646

Centeno, Barbara A ............. 749, 1744

Centonze, Giovanni............... 591, 592

Cerato Biderbost, Christelle....... 1840

Cerda, Sandra.............. 376, 386, 1052

Cereceda, Laura ........................... 1418

Cerhan, James R............................ 1363

Cerna, Iva ................................................ 543

Cerutti, Roberta.............................. 1500

Cesari, Matthew ..... 1087, 1097, 1098

Cesarman, Ethel .............................. 491

Cessna, Melissa..................................... 2044

Cha, Eugen K ............................... 939

Cha, Eugene K ......... 847, 1000, 1080

Cha, Eun Jung ............................... 1015

Chabot-Richards, Devon.............. 1506

Chadburn, Amy ................... 1441, 1468

Chadwick, Dianne........................ 1956

Chae, Seoung Wan ............................. 194

Chae, Yang Seok ………............... 1015

Chae, Yang-Seok ............................. 707

Chai, Ming ........................ 1368, 1457

Chajewski, Olga S......................... 474

Chakera, Aron ............................... 1622

Chalabreysse, Philippe .................. 493

Challies, Tracy ………………...... 1739

Chalmers, Zachary .......... 1123, 1699,

1795

Chalouni, Cecile M .................... 2067

Chambers, Setsuko K.....................1110

Chambon, Pierre .............................. 1811

Chan, Alice NH.......................... 1147

Chan, Jackie .................................... 976

Chan, Joanna ................................. 1134

Chan, May P........ 487, 503, 531, 539

Chan, Yiong-Huak ........................ 533

Chandan, Vishal ..... 1643, 1644, 1645

Chandarlapaty, Sarat ..................... 270

Chandramohan, Raghu.......... 34, 1186

Chang, Angela Y ............................. 321

Chang, Anthony .................... 22, 1600

Chang, Bin ....................................1112

Chang, Chung-Che....................... 2083

Chang, Connie Y ............................... 45

Chang, Hsien-Kun............................... 297

Chang, Hsim-Yee ............................. 873

Chang, Kenneth..................... 44, 2030

Chang, Kuang-Jung....................... 2051

Chang, Kung-Chao ……………... 1343

Chang, Martin C........280, 1115, 1116

Chang, Matthew T......................... 939

Chang, Mee Soo................................ 1650

Chang, Michael D ………………... 647

Chang, Tylis Y............................. 2015

Chang, Yen-Chieh ............................ 723

Chang, Yu-Sun ...................... 173, 297

Changou, Chun A.............................. 1168

Channir, Hani I.............................. 1269

Chapman, Jennifer ...................... 110,1312

$1370,1425,1437,1490,1495$

Chapuy, Bjoern ................. 1144, 1379

Charabi, Birgitte W ...................... 1269

Charest, Karry ................................. 1433

Charette, Lori .......................... 124, 125

Charlesworth, Cristine ................... 489

Charoenpitakchai, Mongkon....... 1592

Charon-Barra, Celine ........................ 74

Chastain, Elizabeth ……………....... 1498

Chaterjee, Monica .......................... 1041

Chau, Danielle B................1146, 1196 
Chaubey, Alka ... 71, 508, 1409, 1711, 1804,2031

Chaudhary, Shweta ............ 401, 1529

Chaudhry, Rahman......................... 102

Chaudoir, Catherine .................... 1344

Chaux, Alcides ................................ 883

Chavez, Luis ................................ 2089

Che, James ..................................... 456

Chebib, Ivan....................................... 45

Cheema, Zulfiqar............................. 29

Cheeney, Gregory ................ 1646, 1647

Chehab, Farid............................... 1513

Chen, Athena..................................... 1135

Chen, Beiyun................................ 2005

Chen, Benjamin J.............. 1484, 1524

Chen, Carlitos ................................. 365

Chen, Chun-Liang..................33, 1316

Chen, Congde................................... 484

Chen, Derrick J ........................... 1536

Chen, Dong ....................... 1338, 1383

Chen, Eleanor..................................... 88

Chen, Guilan .................................... 839

Chen, Guoli ……………………..... 1043

Chen, Haiyan................ 838, 839, 1790

Chen, Hao ..............................348, 1110

Chen, Hoe Meng ........................... 1173

Chen, Hsiang-Chun ........................ 1023

Chen, Hui .......... 147, 445, 721, 1957,

2003

Chen, Jei............................................. 1819

Chen, Jey-Hsin ............................... 648

Chen, Jie...................................... 1741

Chen, Jie-Fu .................................. 878

Chen, Jun-song .............................. 1171

Chen, Justin H................................. 1596

Chen, Ken ................................... 1044

Chen, Lei........................................ 1428

Chen, Li-Rong.............................. 1564

Chen, Lina..................................... 1939

Chen, Min .................................... 1926

Chen, Ning Neil ........................... 1569

Chen, Peter ......................................... 649

Chen, Pu................ 1345, 1346, 1355

Chen, Richard ................. 1792, 2052

Chen, Shao-yong ......................... 1078

Chen, Shaoxiong .......... 46, 652, 1020

Chen, Shin-Cheh .................. 173, 297

Chen, Sonja ........................... 47, 1793

Chen, Steven X ……………….... 1495

Chen, Taiying .................................. 976

Chen, Weina ........... 1345, 1346, 1348, $1349,1355,1459,1460$

Chen, Weiyi................................... 1342

Chen, Wen-Chung ......................... 1343

Chen, Wenqian ...............................1111

Chen, Wilson................................. 1043

Chen, Xiaowei.................................. 161

Chen, Xueyan....................... 879, 1531

Chen, Y-B ....................................... 910

Chen, Yao-Tseng .............................. 1856

Chen, Yen-Ying .............................. 134

Chen, Yigu ......................... 1958, 1959

Chen, Ying ......................... 110, 1782

Chen, Ying-Bei............ 847, 939, 940, $1000,1036,1080$

Chen, Yingbei................................ 1028

Chen, Yongxin..................................... 767

Chen, Yuan Y …………………... 1423

Chen, Yuan Yuan ........................... 1329

Chen, Yunn Yi .................................. 121
Chen, Yunn-Yi..... 122, 213, 214, 299 ,

1233

Chen, Zhengming .................659, 660

Chen, Zi........ 1347, 1396, 1419, 1509

Chen, Zongming E............ 650, 1695, 1942,1960

Chenard, Marie-Pierre......... 135, 254, 671

Cheng, Angela..........1140, 1152, 1236

Cheng, Donavan............................ 1186

Cheng, Esther.............. 136, 137, 1270

Cheng, Jason ................................ 1504

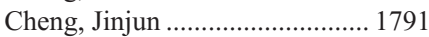

Cheng, Ke ……………................ 1552

Cheng, Liang.......... 46, 651, 864, 865,

$873,941,942,964,968,982,1074$

Cheng, Lin...................................... 880

Cheng, Mingyu .............................. 1597

Cheng, Yu-Wei ............................... 486

Cheng, Yufan.......................1101, 1112

Chennuri, Rohini............................ 367

Cher, Michael L..................... 890, 944

Cheris, Sachica.............................. 138

Cherniack, Andrew ......................... 846

Chernock, Rebecca ............ 574, 1275,

1275,
1323

Cherry, Athena ................................. 1431

Cherukuri, Durga P ..................... 1473

Chesser, Jason .............................. 1648

Chetritt, Jerome...................... 254, 493

Chetty, Runjan.................................. 747

Cheung, Annie N................................... 1147

Cheung, Grace.............................. 1824

Cheung, Hoiwan H...................... 1857

Chevez-Barrios, Patricia ............. 1553

Cheville, John C......... 959, 999, 1884

Chevreau, Christine............................ 74

Chhieng, David C.............................. 454

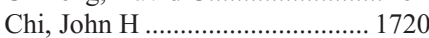

Chi, Zhikai ....................................... 652

Chia, Noel .................................... 1308

Chia, Stephen KL........................... 281

Chiang, Sarah ................................ 1133

Chida, Koji ............................................ 333

Chien, Hui-Ping ............................... 173

Chinea, Felix M ............................. 969

Chinnaiyan, Arul M .......... 988, 1065,

1066

Chiosea, Simion I.... 604, 1271, 1272, $1310,1314,1316$

Chioureas, Dimitrios .................... 1497

Chirieac, Lucian........................... 1899

Chitale, Dhananjay A .......... 107, 186, 1075, 1076, 1085, 1089, 1961

Chiu, April........................ 1455, 1476

Chiu, Charles.................................... 869

Chiu, Hak Fai ................... 1097, 1098

Chivukula, Mamatha...................... 129

Chkheidze, Rati.............................. 653

Chmielecki, Juliann........... 1699, 1785,

Cho-Vega, Jeong Hee............. 494

Cho, Eun Yoon .................................. 139

Cho, Hyun Yee ............................. 1015

Cho, Margaret ............................. 654,815

Cho, Min-Sun............................... 1015

Cho, Nam-Yun ................................... 753

Cho, Soo Youn ............................... 139

Cho, Sooyoun................................ 2048

Cho, Yong Mee .............................. 459, 1015

Choe, Ji-Young.

522
Choe, Jin

.

Choi-Kim, Lydia ........................... 273

Choi, Chan ................................... 1882

Choi, Da Hoon ................................... 2059

Choi, David B …………………... 1728

Choi, Dong Ho ........................ 706, 1691

Choi, Eunice K............................. 1649

Choi, Euno ..................................... 1650

Choi, Gheeyoung .......................... 1015

Choi, Hyejeong ....... 773, 1759, 1767,

1784

Choi, Joon H ………………….... 1742

Choi, Kyung Un ...................... 627, 760

Choi, Misun......................... 139, 1743

Choi, Seoung Ho ........................... 1743

Choi, Sung-eun ................... 576, 2032

Choi, Won-Tak …......................... 1651

Choi, Woonyoung .................. 891, 935

Choi, Yeong Jin .............................. 1015

Choi, Yoon-La ..................... 828, 1858

Choi, Yu-Deok ............................. 1859

Choo, Shoa-Nian............................... 533

Chopra, Pradeep ............................. 1728

Chopra, Shefali ............................... 573

Chorzalska, Anna .......................... 1378

Choudhary, Ajuni .......................... 1976

Choung, Hae Yoon G ..................... 1598

Choung, Ho-Kyung........................ 522

Chow, Chi-Wan ............................ 1044

Chow, Christine.................... 281, 1751

Chow, Kin Long........................... 1147

Choy, Bonnie......................... 852, 882

Christensen, Brock C ….............. 1802

Christensen, Paul.......................... 1553

Christie, Alana................................. 914

Christova, Rossitza ...................... 1002

Chrysostomou, Chrysostomos ... 1081

Chu, Andrew ......... 1345, 1348, 1349,

1355

Chua, Jamie S............................... 1612

Chuang, I-Chieh............................... 75

Chuang, Wen-Yu .................... 173, 297

Chung, Catherine ...................... 33, 65

Chung, Doo Hyun ......................... 1876

Chung, Hyun Cheol ........................ 312

Chung, Jin-Haeng ............................... 1920

Chung, Leland WK .......................... 878

Chung, Min Sung ............................ 101

Chung, Stephen S......................... 1478

Chung, Yumin ............ 101, 706, 1691

Church, Deanna M........... 1792, 2052

Churchill, Hywyn.......................... 1459

Chute, Deborah J.......................... 588

Cibas, Edmund ..... 403, 458, 599, 613

Cicenia, Joseph C......................... 481

Cieply, Kathleen M............. 354, 1414

Cimic, Adela ………………….... 1138

Cimini, Beth................................... 2040

Cimino-Mathews, Ashley ... 239, 261,

293, 1999

Ciomek, Natalie ............................ 734

Cipriani, Nicole A ...... 575, 582, 1309 ,

1311,1925

Clark, Ashlynne.............................. 496

Clark, Jennifer L ...............................1113

Clark, Jonathan R... 1292, 1293, 1308

Clark, Michael J ............... 1792, 2052

Clark, Peter E ............. 892, 925, 1021

Clarke, Loren E.............................. 505

Clarke, Michael............................. 2007

Clarke, Richard R......................... 2062
Clavero, Omar OC ............. 871, 1194

Claxton, David .............................. 1472

Clay, Michael ...................................... 48

Clayman, Gary L............................. 612

Clayton, Erica F ………………......... 687

Clayton, Frederic................................... 655

Clayton, Helen ................................ 437

Clemens, Mark W ............ 1368, 1457

Clement-Kruzel, Stacia ................ 1812

Cleven, Arjen HG......................... 1387

Clevenger, James ............................... 1384

Click, Sarah................................. 1833

Cline-Kabba, Edith ...................... 1487

Clinton, Lani K ....................656, 1114

Cloe, Adam ...................................... 368

Cloetingh, Daniel ............................ 1350

Close, Brett E ............................... 1925

Clurman, Bruce ................................. 879

Cocchi, Stefania ............................... 86

Cochand-Priollet, Beatrix ............... 593

Cocker, Myra .............................. 2025

Cocker, Rubina............................... 924

Cockerell, Clay J............................ 505

Cocks, Margaret............................... 883

Coffey, Amy ................................. 1667

Coffey, Donna ................... 1970, 2037

Coffin, Cheryl M............................ 1971

Coffman, Brittany ........................ 1340

Cohen, Cynthia ... 260, 349, 350, 429, 435, 453, 580, 1283, 1621, 1989 ,

2029

Cohen, David .................... 1148, 1941

Cohen, Debbie L ………………....... 589

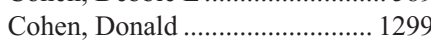

Cohen, Eric …………………….... 1617

Cohen, Jarish............ 657, 1626, 2033

Cohen, Mark L ............................... 1708

Cohen, Michael B ........................ 1955

Cohen, Stephanie M........................ 193

Cohn, David E............................. 1148

Cohn, Ian S................................... 712

Coindre, Jean-Michel .................. 73, 74

Coker, Ahmet ............................... 1692

Colasse, Élodie ..................................... 38

Cole, Bonnie ................................ 1962

Coleman, Jonathan A ................... 1000

Coleman, Joshua ……….............. 1829

Colgan, Terence .................1115, 1116

Collazo Lorduy, Ana .......... 877, 1551

Collingwood, Robin....................... 884

Collins, Brian T....................... 357, 411

Collins, Jennifer A......... 345, 369, 370

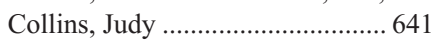

Collins, Katrina .............................. 140

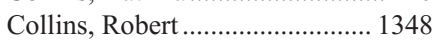

Collinsworth, Amy ......................... 647

Colvin, Robert B ........................... 1585

Comanescu, Maria ........................ 287

Comperat, Eva................... 873, 1062

Compton, Frances .................... 141, 197

Compton, Margaret ................. 7, 142

Conces, Miriam............................. 1832

Conde Da Silva Fraga,

Emmanuele ................................. 671

Condello, Vincenzo................................. 571

Condom-Mundo, Enric ................ 1010

Conlon, Kevin C .............................. 658

Conner, James ................................ 1180

Conners, Amy L ..................... 229, 305

Conrad, Rachel................................ 456

Contos, Melissa J ............................. 30 
Contra, Jessie …………………..... 1962

Contreras, Alejandro ................... 2039

Conwell, Darwin L....................... 2072

Coogan, Alice.................................... 469

Cook, James R .......... 151, 152, 1372,

$1445,1510,1511$

Cooney, Anthony.............. 1191, 2034

Cooper, Kumarasen... 96, 1213, 1298 ,

1982, 2087

Cooper, Lauren............................. 1134

Cooperberg, Matthew R................. 993

Cope, Leslie ................................... 205

Copeland, William C.................... 2079

Coppa, Joregelina................. 591, 592

Coppola, Domenico ... 749, 816, 1744

Corben, Adriana D ............... 252, 277

Corbman, Melanie.......................... 103

Cordeiro, Ana ................................... 1907

Cordero, Anibal.............................. 276

Cordon-Cardo, Carlos ........ 877, 1551

Cornejo, Kristine M .... 509, 525, 885, 982

Cornell, Lynn D ............... 1586, 1618

Corney, David ............................... 1793

Cornic, Marie .................................... 38

Cornish, Toby C ....................... 4, 806

Corpus, George ………………..... 2083

Corrado, Andrea .............................. 332

Correa, Arlene M ......................... 623

Correa, Hernan ................................. 945

Correa, Nathalie QPC ................. 2038

Corrigan, Mark............. 126, 127, 128

Cortes, Andrea................... 199, 2049

Cortes, Jorge.................................. 1509

Corvera, Carlos U ...................... 1755

Cosar, Ediz F...... 509, 885, 982, 1523

Cosimi, A Benedict ....................... 1585

Cosio, Fernando G ...................... 1586

Coss, Dylan ..................................... 1700

Cossa, Mara................................... 1904

Costa, Helio A .................... 1758, 1807

Costa, Larissa BE.................. 866, 867

Costes, Valerie.............................. 1273

Costigan, Danielle.... 658, 1099, 1317

Costinean, Stefan ...... 789, 790, 1351,

1352

Cote, Gilbert........................................ 612

Cote, Michele L............................... 251

Cotelingam, James ....................... 1344

Cottrell, Catherine E ......... 601, 1601,

1697,1914

Couce, Marta............................... 1708

Coughlin, Geoff ................................. 946

Couloigner, Vincent ..................... 1834

Couture, Christian ........................... 336

Covinsky, Michael ............... 141, 197

Cowan, Morgan.......... 886, 887, 1004

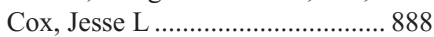

Cox, Roni M........................ 907, 957

Cracchiolo, Jennifer ....................... 1320

Craig, Ryan .................................. 1353

Cramer, Harvey M .............. 344, 2050

Crane, Genevieve M ........ 1376, 1429

Crapanzano, John P....................... 460

Crawford, James M....................... 2015

Crawford, Susan E ......................... 767

Creighton, Chad ................. 846, 1044

Crespo, Mateus ................. 1001, 1002

Cristobal, Eva................................ 1197

Croce, Carlo ............... 789, 790, 1351

Croce, Sabrina .............................. 1207
Crook, Shannon............ 144, 219, 286

Crowe, Deborah O ....................... 1603

Crowley, Jacob P.......................... 1221

Cruise, Michael ....... 1657, 1663, 1664

Crukley, Cathie ............................ 2025

Crum, Christopher P ........1118, 1144, $1157,1180,1234,1247,1248$,

1252

Crumley, Suzanne M....................... 371

Cruz, Ofelia................................... 1844

Cuatrecasas, Miriam ................... 630, 2042

Cubilla, Antonio L....... 870, 871, 909,

1035

Cuda, Jacqueline ...............................355

Cui, Fulan..................................... 1610

Cui, Isabelle ..........................659, 660

Cui, Min ............................................ 661

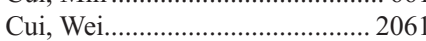

Cui, Xiaojiang .................................. 309

Cui, Xiaoyan ...................................... 147

Cunha, Isabela W ...... 886, 887, 1004,

1062

Cunningham, Mark T.................. 2061

Cunningham, Scott A ................... 1536

Cupo, Marco …………………........ 217

Curless, Kendra...................... 46, 873

Curran, Emily.................................. 1504

Currie, Catherine ........................... 2025

Curry, Jonathan L ......... 488, 490, 523, $530,534,535,540$

Curtis, Mark T.............................. 1537

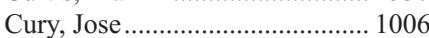

Cuzick, Jack .................................. 863

Cyrta, Joanna..... 23, 889, 1024, 1056,

$\begin{array}{ll} & 2035 \\ \text { Czader, Magdalena } & 1354\end{array}$

Czapiewski, Piotr ............................ 1862

Czerniak, Bogdan.................. 891, 935

Czerniak, Bogdan A ............... 846, 923

Czuchlewski, David ......... 1486, 1508

D'Alfonso, Timothy .............. 136, 137

D'Arcy, Tom ..................... 1191, 1243

D'Cruz, Anil..................... 1288, 1297

D'Costa, Zenobia .......................... 1779

Daaboul, MHD Fayez .......... 273, 398, $890,944,1090,1217$

Daaboul, Mohammed F .............. 1083

Dabbs, David....... 166, 278, 279, 288, $996,1238,2010$

Daber, Robert ................................ 1796

Dacey, Elizabeth ........................... 372

Dacic, Sanja ............ 605, 1891, 1892

Dadhania, Vipulkumar ........ 891, 935,

Dahia, Patricia .............................. 596

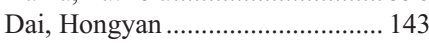

Daignault, Stephanie ..................... 183

Dal Cin, Paola ............ 742, 933, 1133 ,

1508

Dalbagni, Guido .................... 847, 939

Dalimier, Eugenie ........................ 2090

Dalland, Joanna............. 144, 219, 286

Dallimore, Nick............................... 437

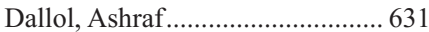

Dalton, Leslie ..................................... 145

Dalvi, Siddhartha ...... 146, 300, 1470,

1860

Damjanov, Ivan .................... 81, 1027

Damjanov, Naveena ...................... 1639

Dancsok, Amanda R............................ 50

Daneshbod, Yahya........................ 1526
Dangott, Bryan J

549

Daniel, Tanisha....................... 783, 784

Daniels, Brianne H................. 51, 506

Daniels, Gilbert ................................. 441

Daniels, Lauren .......... 351, 636, 1771

Daniels, Molly.............................. 1214

Dantey, Kossivi E............................. 52

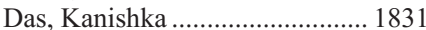

Das, Kasturi............................977, 979

Das, Ronnie.................................... 2070

Dasaraju, Sandhya........................ 1988

Dasarathy, Srinivasan.................. 1662

Dasari, Surendra...... 319, 1363, 1590 ,

Dastani, Zari.............................. 901

Datnow, Brian ................................ 121

Daum, Ondrej................... 1010, 1027

Davanlou, Masoud .......................... 1526

Dave, Sandeep............................. 1354

Davenport, Robertson .................... 467

Davey, Diane D ............................. 461

Davicioni, Elai ................................. 906

Davick, Jonathan...................495, 1117

David, Marjorie............................... 552

David, Stephanie N ....................... 892

Davidson, Ben............................. 1168

Davidson, Benedicte ....................... 110

Davidson, Bennett........................ 2036

Davies, David L .............................. 561

Davies, Michael A......................... 2064

Davis, James ................................. 1947

Davis, Jessica L.............................. 1962

Davis, John W .............................. 1061

Davis, Noa...................................... 373

Dawidek, Theresa.......................... 206

Dawson, Heather........... 662, 663, 664

Dawson, Heather AE....................... 675

Daya, Dean.................................... 1219

Dazza, Marie .................................... 665

de Abreu, Francine ............ 1770, 1787

de Angelis, Camila M .......... 866, 867

De Baere, Thierry........................ 1822

De Bono, Johann S... 976, 1001, 1002

De Braud, Filippo.............................. 592

De Brot, Marina .............................. 711

De Bruijn, Ino ................................. 268, 1250

De Cotiis, Daniel.......................... 1134

De Filippo, Maria.......................... 1185

De Filippo, Maria Rosaria............ 250,

1250

De Jesus-Acosta, Ana....................... 812

De la Oliva, Jorge.......................... 1289

De Las Casas, Luis............................... 417

De Maglio, Giovanna......................111

De Marzo, Angelo M .......... 849, 930 $931,970,1703$

de Muga, Silvia ..................... 893, 947

De Napoli, Luigi ............................... 571

De Nictolis, Michele ................... 1195

de Peralta-Venturina, Mariza .... 1045 ,

de Riese, Werner T 1047

de Sanjose, Silvia ............... 871, 1194 de Torres, Carmen ......................... 1844

De Torres, Ines ..................................... 894

Deavers, Michael ........................ 2037

Decker, Paul A.............................. 1725

Decouvelaere, Anne-Valerie .......... 74

Dee, Anne........................................ 989

Deeken-Draisey, Audrey ................ 666

Deftereos, Georgios
Degnim, Amy....... 119, 132, 133, 204

DeGraff, David J ......................... 1043

Dei Tos, Angelo P .............. 84, 86, 87

Dei Tos, Angelo Paolo ..................... 56

Dei tos, Paolo ...................................... 82

Deininger, Michael................................ 1353

DeJoseph, Maura........................... 1232

DeJulio, Todd ...................... 577, 1652

Del Bigio, Marc ............................. 1723

del Pino, Marta............................... 1220

Del Pozo, Maeva.............. 1738, 1772

Delacruz, Victor .................... 375, 387

Delahunt, Brett... 874, 875, 946, 1074

DeLaMonte, Suzanne.................. 1835

DeLelys, Michelle E .................... 1974

Delgado, Salvadora ......................... 2042

dell'Aquila, Marie........................ 1731

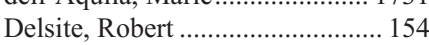

Dema, Alis....................................... 287

Demaria, Sandra............................. 275

Demas Alvares Cabral,

Monica M

Demicco, Elizabeth G ...53,61,1284,

1295

Demichelis, Francesca ....... 889, 1828

Deng, April................. 502, 509, 525

Deng, Fang-Ming ........ 185, 616, 881, $949,966,977,978,979$

Deng, Xiaobing ............................... 99

Denison, Lori ................................... 204

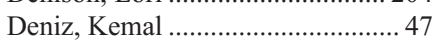

Dennis, Jake .................... 1346, 1355

Dennis, Katie................................... 255

Deplechain, Claude ......................... 493

Desai, Neil............................. 847, 939

Desai, Parth .......................... 1492, 1525

Desai, Sangeeta ..................... 249, 995

Deshpande, Anita ...................... 376, 386

Deshpande, Vikram....... 45, 626, 667,

782, 796, 797, 821, 831, 1135, 1227, 1306, 1336, 1387, 1546, 1630, $1653,1747,1821$

DeSloover, Daniel........................ 1796

Desouki, Mohamed M ........ 188, 189, $190,1192,1210$

Dettloff, Jennifer L........... 1274, 1744

Detweiler, Claire J...............................68

DeVilla, Vanessa ................................ 369

Devins, Kyle ................................. 1963

Devlin, Sean.................................. 1466, 1478

Dewar, Rajan..................... 1584, 2006

Dey-Rao, Rama............................ 2060

Dhall, Deepti ................................. 1669

Dhamne, Sagar ................................. 147

Dhanasekaran, Saravana M......... 1066

Dhand, Abhay ...................................... 1571

Dhani, Neesha ........................ 747, 1774

Dhar, Manjima .................................. 456

Dhillon, Jasreman ..... 895, 1022, 1023

Dhingra, Sadhna........................ 1633

Dhir, Rajiv........... 354, 355, 859, 996,

1017,1018

di Capua, Daniele M .................... 1762

Di Carlo, Dino................................... 456

Di Martino, Julie ............................ 1672

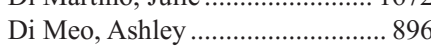

Di Napoli, Arianna ........... 1368, 1457

Diaconescu, Elena D ...................... 669

Diamandis, Eleftherios P...... 675, 896

Diamond, Eli

1448 
Dias, Leonardo .............................. 1737

Diaz-Cano, Salvador ............ 578, 897,

1794

Diaz-Insua, Mireya ...... 955, 956, 985

Diaz, Lorena................................. 2077

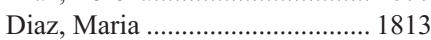

Diaz, Sherley ........................ 49, 2073

DiBardino, David........................... 460

Dibe Gondim, Dikson ................... 1275

DiCarlo, Christina ........................ 1933

Dickerson, Jane ............................. 1962

Dickler, Maura ............................... 201

Dickson, Brendan C .......................... 54

Diebold, Jacques .......................... 1454

Diez-Valle, Ricardo..................... 1706

Dighe, Manjiri K............................. 374

DiGiuseppe, Joseph A ................. 1509

Dillon, Deborah A ............................. 223

Dillon, Jessica L ............................. 148

DiMaio, Michael A.......................... 413

DiMarco, Michelle....................... 898

Dimitrova, Nevenka.......... 308, 1539 ,

1571

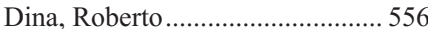

Ding, Qiang...................... 1034, 1057

Ding, Qingqing ............ 149, 150, 476

Ding, Xiangdong......... 472, 482, 1256

Ding, Xianzhong ....... 838, 839, 1790,

1975,1991

Dinney, Colin ........................ 891, 935

Dintzis, Suzanne M....................... 266

Diolaiti, Morgan................................... 2040

Diolombi, Mairo L.... 899, 994, 1029,

1159,1160

Dip, Nelson ....................................... 998

Dislich, Bastian .............................. 670

Dispenzieri, Angela......................... 319

DiStasio, Marcello M.................... 692

Dittman, David............................... 1758

Ditzel, Helena M...............1118, 1234

Divatia, Mukul ............................. 1012

Djordjevic, Bojana .....860, 861, 1119, $1120,1141,1153,1161,1200$

Do, Minh ......................................... 215

Do, Sung-Im.................................. 194

Do, Trevor ........................................ 1774

Doberer, Daniel ............................. 1930

Dobson, Richard ................. 897, 1794

Dockter, Travis J .......... 229, 230, 305

Dodd, Leslie G............................. 1879

Dogan, Ahmet ............ 64, 1375, 1382, $1448,1474,1475,1481,2062$

Dogan, Snjezana .............. 1321, 1880

Doganavsargil, Basak.................... 766

Doglioni, Claudio............................. 609

Dolan, Michelle M..................... 1261

Dolatkhah, Shahaboddin ............. 1054

Dominoni, Francesca ...................... 592

Donahue, John E ........................ 1728

Donaldson, Alana R ............. 151, 152

Donati, Davide …………………...... 86

Done, Susan J............................... 1824

Dong, Fei................. 752, 1248, 1898

Dong, Jianli ................................... 1687

Dong, Joanna.................................. 1284

Dong, Kun.................................... 1681

Dong, Yan....................................... 185

Donovan, Michael J ....................... 908

Donson, Andrew M.................... 1839

Dorfman, David M..... 94, 1493, 1494

Dorreen, Alastair P........................ 732
Doss, Denaha J............................. 1422

Doucet, Laurent................................ 671

Douglas, Greg ………………….. 1120

Downes, Michelle ................ 900, 1067

Downing, James ........................... 1791

Downs-Kelly, Erinn .... 130, 151, 152, $175,621,728,1949$

Doxtader, Erika E.................. 377, 378

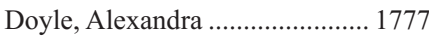

Doyle, Leona.................................. 1757

Doyle, Leona A ………............... 55, 77

Doyle, Maria M............................. 1640

Doyle, Scott.................................. 1280

Dragnev, Konstantin H................ 1928

Drake, Charles..................... 856, 1053

Drakos, Elias ................................. 1497

Dresser, Karen.................... 1484, 1524

Drogowski, Laura M.................... 1582

Druffel, Elizabeth M .................... 1536

Dry, Sarah M................. 47, 79, 1538

Du, Xiang .......... 651, 751, 805, 1245

Du, Yunzhi ...................................... 312

Duan, Xiuzhen ......................147, 1790

Duan, Xuejing .................................. 326

Dube, Valerie........... 1087, 1097, 1098

Dubeau, Louis ............................... 1092

Dubielecka, Patrycja M................ 1378

Duboy, Jon ................................. 1575

Duc, Arnaud ................................ 2090

Ducar, Matthew ………………........59

Ducatman, Barbara ....................... 1215

Dudley, Beth ................................... 1775

Dudley, Jonathan........................... 442

Duffield, Amy S ....... 768, 1376, 1429

Duggan, Maire ..............................1111

Duke, Linnea................................. 901

Dulau Florea, Alina ........... 1356, 1357

Dumitriu, Bogdan ............. 1357, 1405

Dumur, Catherine I .... 30, 1730, 1990

Dunatov, Ana................................ 1010

Dunbar, Cynthia ............................ 1357

Duncan, Sheila .................................... 2064

Duncan, Virginia E...................... 1358

Duncavage, Eric J ....... 514, 601, 684, $1330,1697,1914$

Dundas, Nicola.............................. 2037

Dunlap, Jennifer................ 1449, 1833

Dunn, Andrew LJ ............................ 685

Dunn, Ian F ................................ 1696

Dunn, Kenneth .............................. 2086

Dunne, Richard F ............................ 640

Dunseth, Craig ............. 8, 1745, 1778

Dunya, Gabriel............................ 1276

Duose, Dzifa Y .............................. 2058

DuPont, Barbara R ............... 508, 1711

Durbin, Eric................................... 1926

Dureau, Zachary J ....... 260, 902, 918 ,

1964, 1965

Durham, Benjamin H.................. 1448

Durkin, Lisa M............................... 1445

Dursun, Nevra .................... 1763, 1764

Dussaq, Alex ................................ 1554

Dutta, Anupurva ........................... 1701

Dyatlov, Artem ............................ 1851

Dyhdalo, Kathryn S ........................ 153

Dykoski, Richard ............................ 20

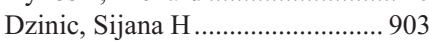

Eastwood, Daniel C ............. 943, 954

Eberhart, Charles G...................... 1703

Eble, John...............................941, 942

Eble, John N........ 873, 874, 875, 968 ,
1074

Echarri, Maria Eugenia .................. 421 Echeveste, Jose I ............... 421, 1706 Eckel-Passow, Jeanette ................ 1725 Ecobichon-Morris, Anne................ 361 Edelweiss, Marcia ........ 154, 198, 250, 259,268

Edgar, Mark A .................................... 48

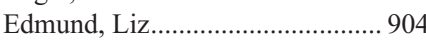
Edwards, Joshua.......................... 1262

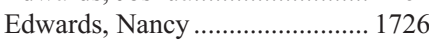

Edwards, William D........................ 314

Egele, Caroline ................................ 135

Egelhoff, Thomas T...................... 1800 Egevad, Lars ................................. 946

Eggener, Scott .............................. 852

Eguchi, Susumu ............................ 227

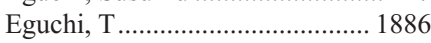

Ehrisman, Jessie A ....................... 1121

Eickhoff, Jens........................................ 1451

Eigsti, Renee ................................. 496

Einhorn, Lawrence H .................... 941

Eklund, Claire M.............................. 390

Ekmejian, Rafael............... 1292, 1293

El Hag, Mohamed ........ 379, 391, 606,

1122,1924

El Jamal, Siraj ...................................... 1498

El Naderi, Samah ......................... 1263

El Naili, Reima............................... 380

El-Achkar, Tarek ........................... 2086

El-Halaby, Amber A ........................ 303

El-Mofty, Samir K ........... 1275, 1286,

1323

El-Rayes, Bassel .................. 772, 773

Eladl, Ahmed E ………………... 1359

Eldersveld, Jordan M .................... 348

Elemento, Olivier........ 23, 889, 1024, $1786,1816,1828,2035$

Elenitoba-Johnson, Kojo.............. 1313

Elenitsas, Rosalie ........................... 505

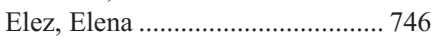

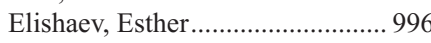

Elkin, Eric ................................... 1214

Ellenburg, James L......................... 922

Ellenson, Lora H .......................... 1179

Elliott, Robin M ............................ 958

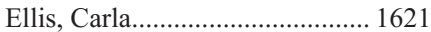

Ellis, Carla L ................................ 1609

Ellis, Ian O ......... 160, 195, 196, 242,

243,250

Elmore, Joann ...................................... 116

Elsayed, Ahmed Ali ..................... 1359

Elshaikh, Mohamed A.................... 1217

Elsheikh, Tarik M............................ 378

Elson, Paul .................................. 1800

Eltoum, Isam ................................ 367

Elvin, Julia A....... 146, 241, 271, 528, $611,701,842,1025,1123,1174$, $1260,1287,1505,1699,1785$ $1795,1853,1927$

Ely, Scott ..................................... 1360 Elyamany, Ghaleb ........................ 1361

Emanuel, Patrick ............................. 506

Emens, Leisha ................................ 293

Emerling, Brooke .............................. 1816

Enderling, Heiko ................................ 816

Endo, Itaru.................................... 1738, 1772

Eng, Charis........................................ 1173

Eng, George ................................. 1653

Eng, Ken .......................... 1786, 1828

Engelberg, Jesse A......................... 121
England, Jonathan ............................ 672

Ennis, Darren ................................ 1100

Epari, Sridhar ................................ 1701

Epstein, Jonathan I ...... 872, 899, 927 , 934, 970, 975, 980, 991, 994, $1007,1008,1009,1028,1029$, 1030, 1047, 1077, 1159, 1160,

Erbarut Seven, Ipek....................... 1767

Ercoli, Giulia................................. 1865

Erickson, Lori A.......... 500, 529, 714,

936, 937

Erlich, Rachel.............................. 1505

Esebua, Magda ................................ 476

Eshleman, James R ............. 984, 1854

Esmaeli, Bita ....................... 534, 1705

Esnakula, Ashwini K.......... 235, 1648

Espinal, Paula.................................. 1206

Esposito, Michael J ..................... 1563

Essendrop, Robyn .......................... 1945

Esserman, Laura J .......................... 122

Esteller, Manel ................................ 746

Esteve, Roser................................. 1220

Estrada, Monica V.......................... 155

Estrella, Jeannelyn S ..................... 623

Eszlinger, Markus ........................... 458

Etman, Ali

1558,1584

Ettel, Mark ........................ 1629, 1654

Euscher, Elizabeth D........1124, 1125, $1178,1209,1253$

Evans, Andrew ....... 1019, 1033, 1582

Evasovich Swenson, Maria........... 452

Everett, Rhonda............................. 393

Eviston, Timothy J ............ 1292, 1293

Ewalt, Mark D............................. 1486

Ewanowich, Carol....................... 1139

Ewaz, Abdulwahab ........................... 381

Eze, Ogechukwu .............. 1629, 1654

Fabbri, Alessandra............ 1903, 1904

Faber, Erin...................................... 911

Factor, Rachel E.......... 115, 130, 164

175,1949

Fadare, Oluwole...... 262, 1210, 1948 ,

$\begin{array}{ll} & 2009 \\ \text { Fajardo, Laura P } & 2038\end{array}$

Falconer, Henrik ................................. 1106

Falconieri, Giovanni ....111, 497, 498 ,

1294

Fallon, John T .... 69, 308, 854, 1159, $1160,1539,1571,1851,1852$

Faltas, Bishoy............ 23, 1024, 1786

Falzarano, Sara..................... 905, 989

Fan, Cathy ……..................1143, 1218

Fan, Fang............. 143, 255, 265, 364

Fan, Guang ................................... 1449

Fan, Xuemo (Sean) ........................ 202

Fan, Yao-Shan ................... 1490, 1825

Fanburg-Smith, JC ................................... 695

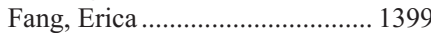

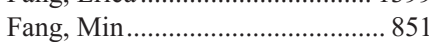

Fang, Yisheng V........... 188, 189, 190

Fanucchi, Michael............ 1851, 1852

Faquin, William C....... 428, 431, 441, $468,1302,2041$

Farag, Rosemary ................... 379, 391

Farah, Nadine................... 1191, 1243

Farahani, Navid.................... 1555, 1575

Faraj, Sheila .................................. 906

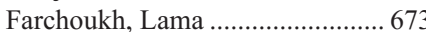

Farell, James J ............................................ 813

Faria, Andre M ................................ 617 
Farmer, Peter..................... 1143, 1218

Farooq, Fahad ................................ 1469

Farooq, Taliya …………………... 1539

Farooqi, Midhat S ........................ 1796

Farooqui, Mohammed.................. 1507

Farris, Alton .................................. 1621

Farris, Alton B.................... 777, 1609

Faruqi, Asma Z............................ 1229

Farver, Carol ..................... 1889, 1895

Fasani, Roberta .......................... 1813

Fassina, Ambrogio ..............................56 556

Fata, Cynthia ................................. 674

Fatheree, Lisa A .............................. 1331

Fathi, Amir T................................. 1387

Fatima, Huma................................ 1596

Fatima, Nazneen ........... 382, 383, 430

Faulkner-Jones, Beverly E .......... 565,

1556,1599

Faust, Phyllis L .......................... 2079

Favazza, Laura .......... 107, 186, 1075,

1076

Fazeli, Roghayeh.......................... 1916

Fazli, Ladan.......................... 993, 1060

Fazlollahi, Ladan......................... 1667

Feany, Mel............................................ 1732

Feasel, Patrick C …............... 486, 507

Febbo, Phillip G ................... 868, 989

Fedda, Faysal A.............................. 907

Fedeli, Franco ................................ 1861

Federman, Noah............................... 79

Fedor, Helen .......................... 906, 970

Fedorchak, Kyle......... 611, 1260, 1287

Fedoriw, Yuri................................. 1362

Feeley, Linda....... 126, 127, 128, 524 .

1265

Feeney, Nora ................................ 1922

Feilotter, Harriet.............................. 1939

Feldman, Andrew L........... 1363, 1386

Feldman, Rebecca ........................ 1797

Feldmeyer, Laurence............ 488, 535

Feleppa, Ernest J ........................... 2068

Felgar, Ray E.................................... 558

Felicella, Michelle........................ 2092

Felisiak-Golabek, Anna................ 1862

Felix, Ana ...................................... 1126

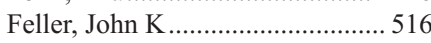

Fellman, Bryan.............................. 1164

Fend, Falko …………………...... 1483

Feng, Felix Y.............................. 1065

Feng, Tian .................................... 1655

Feng, Zheng .................................. 1101

Feng, Ziding ....................... 993, 1060

Fennelly, David .

....... 680

Feratovic, Rusmir.............. 1580, 1581

Fereidouni, Farzad ............ 1712, 2053

Fernandes, Helen....... 347, 693, 1817,

1901,1944

Fernandes, Viviane T .................. 1641

Fernandez del-Castillo, Carlos... 1757

Fernandez Nestosa, Maria Jose... 870,

Fernandez-Nestosa, Maria

Jose......................................... 1035

Fernandez-Pol, Sebastian........... 1364,

1966

Fernandez-Rodriguez, Juana........... 49

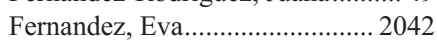

Fernandez, Gerardo........................ 908

Fernandez, Hugo F........................ 1514

Fernandez, Pedro L ...................... 2042

Fernandez, Susan .

353
Fernandez, Susan V.......................... 341

Ferrari, Stefano …………….... 82, 86

Ferreira, Ana ………………….... 1001

Ferreira, Joana................................ 1126

Ferreira, Marco ................. 1193, 1952

Ferrero, Stefano.............................. 1865

Ferro, Paola ................................... 1861

Ferrone, Cristina R..................... 1747

Ferry, Judith A.......... 797, 1336, 1546

Ferte, Charles ................................. 1822

Fetique, Dominique......................... 135

Feuchtinger, Anette ....................... 206

Ffrench, Brendan........................... 2034

Fidai, Shiraz S ............................. 2007

Fidan-Ozbilgin, Ozlem ......... 362, 384

Fidler, Mary E ............................. 1618

Fiel, M Isabel ............................... 1683

Fielden, Rachel ................................... 9

Fields, Kristina L.......................... 1439

Fields, Tina.......................... 988, 1039

Figueiredo, Ines................ 1001, 1002

Figueroa, Lydia E......................... 2089

Figueroa, Maria................. 1514, 1515

Filipenko, Douglas ....................... 1572

Filleron, Thomas ............................... 74

Finberg, Karin E............................ 1843

Findeis-Hosey, Jennifer........ 624, 640

Fine, Jeffrey L ........ 1555, 1557, 1575

Fine, Samson.............. 939, 940, 1080

Fine, Samson W ....... 847, 1000, 1036

Fine, SW....................................... 910

Fineberg, Susan A .......................... 216, 222

Fink, David ................................... 1277

Finkelstein, Sydney D...... 1818, 2076

Finley, Gene .................................. 1933

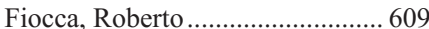

Firan, Mihail ................................... 1348

Firchau, Dennis J ............................... 8

Firestone, Bryan .......... 629, 842, 1082

Fiset, Pierre .................................. 1863

Fishbein, Gregory A........................ 320

Fishbein, Lauren …………………......58

Fisher, Gabrielle ............................. 863

Fisher, Hugh ...................................... 842

Fisher, Kevin E ………………..... 1399

Fisher, Kurt ...................................... 1637

Fisher, Pete................................... 1724

Fitzgibbons, Marissa A................ 1408

Fitzpatrick, Carrie A..................... 1434

Fives, Cassie ....................... 127, 1265

Flake, Darl D.......................................505

Flamerich, Cristina............................ 83

Flanagan, Margaret ........................... 10

Flannigan, Kathleen ......................... 380

Flavin, Richard............................. 2034

Fleischmann, Achim ..................... 919

Flejou, Jean-Francois ....................... 664

Flejter, Wendy L........................... 1058

Fleming, Jason B.................. 726, 750

Fleming, Stewart ........................... 1074

Fletcher, Christopher... 33, 39, 55, 59, $62,77,89,94,932,1285$

Flood, Trevor A.... 860, 861, 862, 950

Flotte, Thomas ........... 500, 529, 2093

Flotte, William .............................. 2093

Fogel, Bradley J ..................................... 685

Fogo, Agnes …………………….... 1613

Fogo, Agnes B....... 1592, 1596, 1603,

1608

Fokas, Emmanouil ....................... 1779

Folkerth, Rebecca D.
Folkins, Ann K .................. 1093, 1973

Folpe, Andrew L ....... 36, 37, 63, 100,

2069

Font-Tello, Alba ................... 947, 948

Font, Alba.................. 893, 960, 2055

Fontaine, Daniel G ......................... 338

Fontanilles, Maxime ................... 1809

Foo, Wai Chin ...... 623, 679, 698, 779

Forcucci, Jessica .................... 550, 551

Ford, Sterling E............................... 345

Foreman, Nicholas K ...................... 1839

Forest, Stefanie ............................ 1992

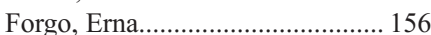

Formenti, Silvia C ...........................2275

Fornander, Tommy .......................... 122

Forsberg, Peter .............................. 1360

Forse, Catherine .................. 675, 1967

Fortuna, Danielle................. 676, 1537

Foss, Francine M......................... 1453

Foster, Richard S ............................ 941

Foucar, Kathryn ............... 1506, 1508

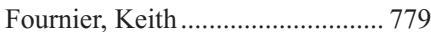

Fowler, Larry J ................................ 1299

Fox, Rosemary ............................... 437

Fox, Sharon E.................................. 1556

Fraass, Benedick ……………….... 309

Fragoso, Maria CV ......................... 617

Fraig, Mostafa M ........ 475, 477, 911,

1498

Frampton, Garrett............ 1123, 1699 ,

1795,1927

Frampton, William ............ 1127, 1968

Frances, Albert .................. 948, 2045

Franceschini, Maria Cristiana .... 1861

Franchi, Alessandro............................ 56

Francoeur, Courtney A .................... 757

Frank, Igor...................................... 999

Frank, Ryan D ...................... 132, 133

Frankel, Arthur E ........................... 1348

Frankel, Wendy ……………........ 2072

Frankel, Wendy L........ 634, 724, 788,

789,790

Franklin, Lindsey ........................ 1095

Fraser, Michael................................. 1067

Frater, John L ................... 1330, 1413

Freed, Natalie S.................. 1365, 1366

Freedman, Alexa ......... 351, 392, 393,

1771

Freedman, Paul .......................... 1261

Freeman, Gordon J........... 1144, 1379

Freire, Javier .......................... 527, 677

Freitas, Leandro LL.............. 866, 867

French, Christopher A ..................... 458

Freud, Aharon ............................... 1335

Freyre, Ashley S ............................ 2007

Fridman, Rafael........................... 1084

Friedman, Kenneth...... 490, 678, 679,

823,912

Friedman, Paul .....................913,914

Fritchie, Karen ....... 35, 36, 42, 46, 71,

100,536

Frizzell, Kimberly ........................ 1503

Frizzell, Norma ................. 1204, 1205

Fromm, Jonathan R.......... 1367, 1531

Frost, Marlene H......... 119, 132, 133,

Frota Lopes, Ana Claudia .......... 1368

Frydenlund, Noah ..................502, 516

Fu, Kai.................. 1351, 1352, 1366

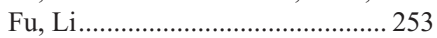

Fu, Liying.................. 915, 952, 1598
Fuda, Franklin ....... 1345, 1349, 1355,

1459,1460

Fuhrer, Kimberly A ...................... 1018

Fujikura, Kohei ................ 1656, 1746

Fujimoto, Junya..... 1873, 1874, 1937,

2057

Fujimoto, Masakazu.................... 1864

Fukatsu, Akitoshi ........................ 1064

Fukayama, Masashi............ 619, 1660,

1661

Fukunaga, Masaharu .........1128, 1129

Fukuoka, Junya ……………….... 1867

Fullen, Douglas R ...... 487, 531, 1805

Fuller, Gregory ............................. 1172

Fulton, Regan............. 157, 499, 1919

Fulzele, Sadanand ....................... 2031

Fumado, Lluis .... 893, 947, 960, 2055

Fumagalli, Caterina...................... 1865

Furlan, Daniela............................... 1500

Furman, Richard R....................... 1373

Furtado, Larissa V.............. 710, 1925

Furth, Emma E ............ 699, 731, 807,

1639,1670

Fusco, Nicola ................................... 1865

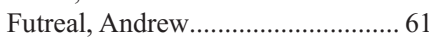

Gaba, Arthur R ................. 1085, 2092

Gabrielson, Edward...................... 1875

Gabril, Manal ............. 916, 953, 1610

Gadde, Ramya......... 917, 1040, 1074,

1085,1369

Gage, Heather M............................ 385

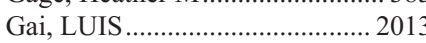

Gaillot, Alain...................................... 493

Gainor, Justin F ........................... 1871

Gajzer, David C............................ 1370

Gal, Tamas .................................... 1926

Galateau-Salle, Francoise ........... 1878

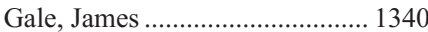

Gales, Jordan M ............................... 1702

Galindo, Julie A............................ 2089

Gallagher, Michael F........ 1099, 2034

Gallan, Alexander J..................... 1600

Gallegos, Marlene .......................... 793

Galletta, Henry ................................ 2056

Gallinger, Steven........................... 1774

Gallo, Marco ……………………........ 569

Gallo, Mathieu ............................. 1273

Galmiche, Louise ......................... 1834

Galsky, Matthew ............................. 877

Galvan, Jose A......................... 663, 670

Gamba, Piergiorgio ....................... 1461

Gambacorta, Francesca ............... 2063

Gambarotti, Marco ....... 56, 82, 86, 87

Gamberi, Gabriella........................... 86

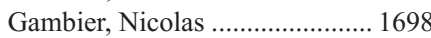

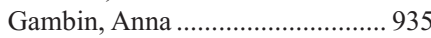

Gamez, Roberto ................. 855, 1576

Gan, Qiong ....................................... 541

Ganapathi, Karthik A ...... 1376, 1405 ,

1992,1993

Gandhi, Manoj ............................... 1323

Gandhi, Nilay .................................. 856

Gandour-Edwards, Regina............. 755

Ganesan, Raji ............................... 1229

Ganly, Ian ......................................... 1321

Gao, Dongxia ................................... 281

Gao, Jing ………………………........ 713

Gao, Juehua........................................ 256

Gao, Kan ………….......... 1292, 1293

Gao, Ling ........................................... 528

Gao, Qi ........................................ 1478 
Gao, Sizhi P.................................... 847

Gao, Yan......................................... 289

Gao, Yuan......................................... 546

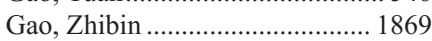

Gao, Zifen ................................... 1450

Gao, Zu-Hua ............................... 1681

Garapati, Manjula ........................... 639

Garces, Jorge .................................. 1603

Garces, Yolanda I .......................... 1911

Garcia-Berbel, Pilar ...................... 677

Garcia-Buitrago, Monica .... 375, 387,

416,778

Garcia-Burillo, Amparo .............. 2073

Garcia-Fernandez, Eugenia......... 1195

Garcia-Grau, Iolanda..................... 894

Garcia-Moliner, Maria ............... 1230

Garcia, Angel ...................... 49, 1197

Garcia, Benjamin A.............. 96, 2087

Garcia, Elizabeth........................... 933

Garcia, Fernando ............................. 103

Garcia, Joaquin J ......................... 1266

Garcia, Julieta ............................ 1917

Garcia, Monica.............................. 1848

Garcia, Rochelle L ........................ 266

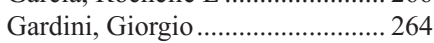

Gardner, Jerad ................................ 552

Gardner, Timothy B ....................... 358

Garg, Karuna .......................1165, 1189

Gariepy, Gille ................................ 343

Garon, Edward ................................ 456

Garratt, John..................... 2020, 2021

Garraway, Levi............................... 889

Garrett-Mayer, Elizabeth............. 1208

Garza, Raquel.............................. 1130

Gascoyne, Randy D ................... 1408

Gasim, Adil ............................... 1612

Gasparinho, Gabriela .................... 1193

Gasparini, Elisa .............................. 264

Gasparotto, Daniela............................ 84

Gatalica, Zoran............................ 1797

Gattuso, Paolo............. 169, 594, 1095

Gauchotte, Guillaume ................. 1698

Gaudioso, Carmelo ...... 188, 189, 190

Gaut, Joseph...................... 1601, 1604

Gawelek, Kara................................ 158

Gay, Laurie....................... 1123, 1795

Gayden, Tenzin ................................ 54

Gayetsky, Vera ................................ 407

Ge, Yimin .......................... 352, 1970

Gedik, Reyhan............................... 386

Geeze, Mary ................................... 1371

Geiersbach, Katherine.......... 175, 716

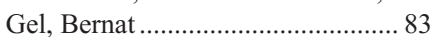

Geller, Rachel....................... 260, 918

Gellert, Lan L.... 892, 925, 945, 1021 ,

2002

Gendusa, Rossella ....................... 2066

Genega, Elizabeth ....... 708, 967, 1996

Genitsch, Vera ................................ 919

Gennaro, Lucas .............................. 277

Gentry, Michael................. 1372, 1511

Geoghegan, Justin ........................... 658

George, Michael............................. 159

George, Tracy I ..... 1331, 1340, 1371 , 1432, 1473, 1506, 1508

Georgescu, Rares .......................... 287

Gera, Shweta ...................... 1629, 1654

Geradts, Joseph …........................... 193

Geraghty, Brian E......................... 1944

Geraghty, Robert ............................. 680

Gerds, Thomas A.......................... 1269
Germer, Jeffrey ............................... 1658

Gerrin, Sean J............................... 1078

Gershenwald, Jeffery E.................. 490

Gershenwald, Jeffrey E................. 2064

Gertych, Arkadiusz ..................... 1045

Gessi, Marco ………………….... 1721

Getz, Gad .......................................... 846

Geyer, Felipe C ... 160, 217, 250, 259, $268,1250,1798,1823$

Geyer, Julia ..................... 1486, 1508

Geyer, Julia T .................... 1373, 1420

Ghaffarieh, Alireza............... 920, 921

Ghai, Ritu ...................................... 169

Ghemrawi, Rose.......................... 1698

Gheorghe, Gabriela ....................... 1333

Ghofrani, Mohiedean ..................... 438

Ghosh, Arunima ........... 681, 768, 771

Ghosh, Kankana .... 1374, 1388, 1389 ,

1390,1391

Ghossein, Ronald .... 614, 1318, 1319

1320
592

Gianduzzo, Troy.................................... 946

Giannico, Giovanna A......... 892, 925, $945,1021,1603$

Giannini, Caterina ............. 1724, 1725

Gibson, Blake................................. 1969

Gibson, Briana R........................... 922

Gibson, Joanna A .................. 645, 646

Gibson, John ................................ 1605

Gibson, Sarah E ..... 1402, 1404, 1414

Gidfar, Sanaz....................................... 1334

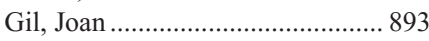

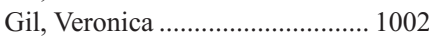

Gilani, Syed........................ 923, 2039

Gilberte, Agnes .............................. 543

Gilks, Blake....281, 1131, 1132, 1139 , $1140,1229,1235,1236,1242$ $1995,2020,2021$

Gilks, C Blake...................1152, 1153

Gill, Anthony J ............. 678, 823, 827

Gill, Ginjeet GK.............................. 12

Gill, Ryan M .......... 1651, 1678, 1688

Gillies, Elizabeth......................... 1946

Gilmore, Hannah......... 153, 158, 269,

1562

Gilroy, Richard............................ 1631

Gimotty, Phyllis A........................ 1514

Gindin, Tatyana ............................. 1401

Ginter, Paula.................................. 1856

Ginter, Paula S ................................. 137

Giordano, Mirella............................ 570

Giordano, Thomas J ..................... 1039

Giri, Dilip ............................ 154, 276

Givertz, Michael M........................ 320

Gjini, Evisa ........................ 1144, 1379

Glass, Benjamin ................................. 117

Glass, Ryan ...................................... 924

Glass, William F............................. 912

Glasser, Daniel ......................... 266, 560

Glavaris, Stephanie ...... 849, 906, 970

Glawe, John.................................. 1344

Gleason Grade 4 Subtype

Collaborative Group................ 971

Gleave, Martin E............................. 993

Gleeson, Noreen.......1177, 1191, 2034

Gleich, Gerald .......................................... 655

Glisson, Bonnie................. 1873, 1931

Glomski, Krzysztof......................... 579

Gnepp, Douglas R......................... 1324

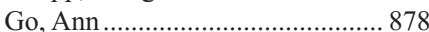

Go, Derek E ……………………..... 456 Go, Heounjeong .................. 459, 1876 Gobbi, Helenice ………………...... 711 Gocke, Christopher D ....... 984, 1429,

1854

Godwin, Andrew K ....................... 143

Goetz, Matthew............ 229, 230, 305

Goez Gutierrez, Elvia...................... 387

Goez-Gutierrez, Elvia ...................... 375

Goh, Gerald.................................. 1453

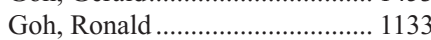

Gokden, Neriman ........ 855, 907, 957,

1062

Gokmen-Polar, Yesim ........... 112, 161 Golardi, Natalia................. 1428, 1465

Gold, Kathryn A ............................ 1931

Goldbach-Mansky, Raphaela ..... 1520

Goldberg, Allison ......................... 1134

Goldblum, John....................764, 765

Goldblum, John R ..... 66, 67, 68, 506,

$763,786,791$

Goldhoff, Patricia.......................... 388

Goldman, Jonathan ........................... 456

Goldsmith, Jeffrey............ 1739, 1958,

2014

Goldstein, David .......................... 1726

Goldstein, Jeffery ................. 892, 925

Goldstein, Jennifer B ..................... 750

Gomez Fernandez, Carmen........... 610

Gomez Macias, Gabriela

Sofia .................................... 1130

Gomez-Fernandez, Carmen .......... 301, $375,387,416,1206,1312$

Gomez-Gelvez, Juan .................... 1375

Gomez-Roman, Javier.................... 677

Gomez, Adam J............................ 389

Gomez, Maria C.................1197, 1197

Gomez, Nerea ................................. 421

Gomolcakova, Barbora .................. 1154

Goncalves, Lucilia ....................... 1193

Gonen, Mithat ................................ 795

Gong, Jerald .................................. 2027

Gong, Shunyou ...... 1376, 1483, 1520

Gong, Yun .................... 414, 468, 485

Gonzalez de Villambrosia,

Sonia ................................... 1418

Gonzalez-Moran, M Asun............. 527

Gonzalez-Vela, Carmen ................. 527

Gonzalez, Blanca .......................... 1483

Gonzalez, Dimitri......................... 2083

Gonzalez, Ivan R.......................... 1917

Gonzalez, Oscar ............................ 2073

Gonzalez, Raul S........ 674, 682, 762,

802,818

Gonzalvo, Pablo ................................ 527

Gooding, William E .................... 1272

Goodman, Abigail .......................... 349

Goodman, Abigail L... 580, 926, 2029

Goodman, Michael...... 351, 392, 393,

$636,773,1771$

Goodman, Steven .............. 352, 1970

Gopal, Purva .... 653, 725, 1646, 1680

Gopal, Satish ............................... 1362

Gopalan, A.................................... 910

Gopalan, Anuradha ..... 847, 939, 940, $1000,1036,1080$

Gordenin, Dmitry A …………….... 846 Gordetsky, Jennifer B......... 884, 922, $991,992,1059$

Gordon, Stuart R ............................ 358

Gorin, Michael A............................ 927
Gorman, Blythe............................ 1970

Goswami, Rashmi ........................ 1377

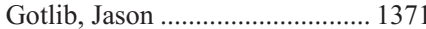

Goto, Hiroaki ................................ 1845

Goto, Keisuke ……………............. 683

Gotoh, Momokazu ... 405, 1063, 1064

Gottlieb, Chelsea E ........................ 162

Gounder, Mrinal M ........................... 64

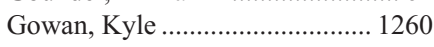

Gowen, Kyle …………………...... 827

Gown, Allen M......... 157, 1919, 2069

Goyal, Bella ....................................... 684

Goyal, Lipika ............................... 1630

Goyal, Rajen .............. 892, 925, 1072

Goyne, Hannah E ............................. 685

Goytain, Angela ...................44, 2030

Grada, Zakaria... 390, 928, 973, 1378,

1547

Graf, Erin H ................................ 1537

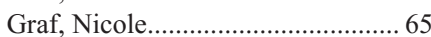

Graham, Alexander ...................... 2027

Graham, Rondell....... 634, 686, 1225, $1657,1658,1665,1666,1684$, 1685,1756

Gramatges, Maria M ................... 1399

Granja, Sara..................................... 617

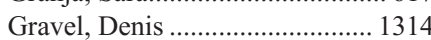

Gray, Arielle........................520, 1987

Gray, Richard J............. 229, 230, 305

Green-Church, Kari.................... 1351

Green, Andrew ..... 195, 196, 242, 243

Green, Anthony ............................. 1018

Green, Peter HR ………...... 712, 1401

Green, Whitney ...................... 681, 771

Greenbowe, Joel................ 1174, 1776

Greenfield, Jeffrey............................. 23

Greenland, Nancy ............... 929, 2040

Greenwood, Michael................... 1799

Greer, Wenda.................................. 1921

Greiner, Timothy C .......... 1352, 1366

Greipp, Patricia ............................. 1657

Griewank, Klaus ……………….... 1721

Griffin, Gabriel K........................ 1379

Griffin, Marie R ….......................... 1192

Griffith, Christopher C........ 472, 482,

Grignon, David J........ 873, 874, 875, $941,942,968,1074,1075$

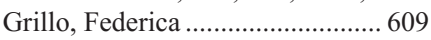

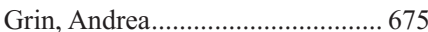

Grizzle, William E ........................ 922

Grogan, Martha ................................ 319

Grogan, Raymon ............................... 575

Grogg, Karen L ........ 163, 1338, 1363

Grondin, Katherine ...................... 1139

Groppi, Diane................................. 2015

Grossman, Stuart.......................... 1703

Groth, John V ................................ 2007

Gru, Alejandro.......... 495, 1335, 1941

Grubbs, Elizabeth G.................... 603

Gruber, Tanja.............................. 1791

Gruenberg, Jessica .......................... 182

Grushchak, Solomiya......... 520, 1987

Gu, Mi J ............................................. 1742

$\mathrm{Gu}$, Xiaoping................................... 161

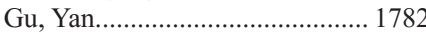

Guajardo, Andrew ..................... 11, 340

Guancial, Elizabeth .........................965

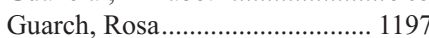

Guardiola, Philippe .......................... 1727

Gubbins, Luke............................... 1099 
Guda, Kishore ………………….... 822

Gueant, Jean-Louis ...................... 1698

Guedes, Liana B................... 930, 931

Guerini-Rocco, Elena........... 160, 217 ,

1865

Guerra, Paula................................ 1193

Guerzon, Godfrey M.................. 1989

Gui, Dorina ……………….... 687, 755

Guileyardo, Joseph M ..................... 24

Guillen, Francisco ……………..... 1706

Guilmette, Julie ............................. 2041

Guimera, Nuria .......... 870, 909, 1035

Guinan, Mairead ............... 2011, 2012

Guindi, Maha ................... 1668, 1669

Guirguis, John ........ 1662, 1663, 1664

Gulati, Rohit............................... 1369

Gulavita, Previn ................... 932, 933

Gulbahce, H Evin.......... 130, 164, 175

Guleria, Indira ............................. 1720

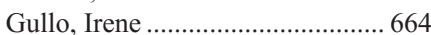

Gultekin, Sakir H ........................ 1830

Guner, Gunes ...... 886, 887, 934, 970,

1004, 1703

Gunning, William T ................... 1625

Guo, Charles C... 891, 923, 935, 1022

Guo, Guangwu ............................... 846

Guo, Hua .............................. 165, 1380

Guo, Huazhang ........................... 1557

Guo, Ming …………..... 371, 414, 485

Guo, Ruifeng................................... 500

Guo, Tao............................................. 283

Guo, Yunquan.................................. 876

Guo, Zhenying ............................. 607

Gupta, Alia ............... 602, 1490, 1495

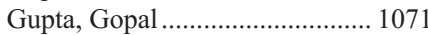

Gupta, Mamta ..............................1118

Gupta, Meenakshi ....................... 2059

Gupta, Nilesh S ... 853, 917, 955, 956, $964,985,1040,1074,1075,1076$

Gupta, Raavi ................................ 1569

Gupta, Rajib ..................... 1602, 1747

Gupta, Ruta ............ 1292, 1293, 1308

Gupta, Sarika.............................. 1381

Gupta, Sounak.... 936, 937, 999, 1800

Gupta, Sudeep ………………….... 249

Gupta, Tejpal....................................... 1701

Gur, Deniz................................... 1382

Gurav, Mamta ................................. 249

Gurbuxani, Sandeep.................... 1504

Gurda, Grzegorz T ......................... 166

Gurman, Jonathan ......................... 1579

Gurpide, Alfonso ………………..... 421

Gurudu, Suryakanth ........................ 799

Gurung, Ananta ........................... 1659

Guseva, Natalya ................................ 60

Gutierrez Barrera, Angelica .......... 147

Gutierrez-Pecharroman, Ana...... 1197

Gutmann, Edward ............... 358, 1787

Guy, Cynthia .................................. 688

Guzman, Grace .................... 793, 2063

Ha, Jennifer P....................... 379, 391

Ha, Sang Yun.................................. 720

Habermann, Thomas ..................... 1363

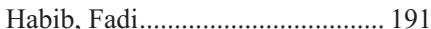

Habib, Robert................................. 1276

Habr, Fadlallah............................ 1789

Habra, Mouhammed A.......... 602, 951

Hacihasanoglu, Ezgi ... 351, 392, 393, $636,826,1748,1764,1771$

Hadd, Andrew ............................. 1804

Hadravsky, Ladislav

.... 81
Haemmig, Stefan.............. 1752, 1780

Hafez, Khaled ................................ 1066

Hafezi-Bakhtiari, Sara................... 747

Hafidi Benammar, Assia ............. 1273

Haga, Hironori .................. 1406, 1913

Hagen, Catherine......... 644, 689, 744,

$\begin{array}{rr}770 \\ \text { Hagiya, Ashley S } & 1558\end{array}$

Hahn, Jamie....................... 1507, 1519

Hahn, Noah M................................ 856

Haider, Irfanullah ............................ 265

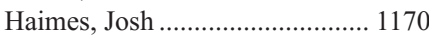

Haines, G K.................................... 1295

Hajdu, Cristina H .............. 1629, 1654

Hajifathalian, Kaveh ...................... 791

Hakam, Ardeshir .......................... 1240

Hakima, Laleh.................................. 394

Hakimi, A Ari .................................. 1036

Hakin, Sofia................................... 1197

Hale, G ........................................ 820

Hale, Gillian L.............................. 1538

Hale, Tracy K ................................... 880

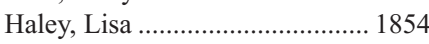

Halimi, Caroline.............................. 1301

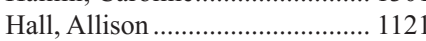

Hall, Dimity ..................................... 431

Hall, Michael................................... 1123

Haller, Florian ................................ 1086

Haller, Jasmine .................................. 552

Haller, Michaela J ........................ 1278

Halpenny, Darragh F .................... 1910

Halperin, Daniel M .......................... 750

Halushka, Marc K ………………..... 340

Hamad, Eman...................................... 337

Hamamatsu, Akihiko...................... 333

Hamasaki, Makoto ............ 1866, 1868

Hameed, Meera ................ 34, 57, 2062

Hameed, Omar .... 283, 892, 925, 945,

$1021,1971,2002$

Hamilton, Ronald ........................ 1722

Hammer, Richard D ........ 1383, 1384,

2028

Hammer, Suntrea ................. 722, 759

Hammerman, Peter ............ 1887, 1900

Hammond, Robert........................ 2025

Hampel, Heather .................... 788, 790

Han, Guangming ..... 167, 1087, 1097 ,

1098, 2071

Han, Misop...................................... 906

Han, Yiping W............................... 712

Hang, Jen-Fan .... 395, 412, 938, 1704

Haninger, Diana M...................... 1559

Hanley, Krisztina Z .... 351, 393, 1771

Hanna, Nasser H ............................ 941

Hanna, Wedad ....................... 167, 2071

Hansel, Donna............ 846, 962, 1032,

1049,1800

Hansen, Colin C .............................. 1617

Hansen, Helen M ......................... 1725

Hansen, Katrine..........218, 1146, 1150

Hanson, Joshua A .............................. 562

Hanson, Paul J................................. 321

Hao, Desiree......................................... 1281

Hao, Suyang ..................... 1341, 1385

Hao, Xueli .............................. 939, 940

Hara, Masatake ............................. 1867

Harada, Shuko............. 168, 257, 1554

Haraldsdottir, Sigurdis ................. 788

Harari, Saul E......................... 941, 942

Harbert, Tracey ............................... 430

Harbhajanka, Aparna.

169
Hardin, Heather A ........ 607, 615, 618 Harding Jackson, Nicholas.......... 1073 Harding-Jackson, Nicholas ......... 170,

Hardisson, David................1195, 1197

Harigopal, Malini......... 124, 125, 248 ,

341,353

Hariri, Lida P................................ 1920

Harmany, Zachary T......... 1712, 2053

Harmon, Michael A...................... 2058

Harmon, Zach ................................. 1121

Harms, Kelly L.............................. 1805

Harms, Paul W ......... 487, 1278, 1805

Harnois, Denise............................ 1686

Harpaz, Noam ....................... 734, 837

Harrington, Alexandra..... 1333, 1446,

1447

Harrington, Kyle ............................ 1599

Harris, Cynthia ............................... 1744

Harris, Hobart W ........................... 1755

Harris, Jason.................................. 1792

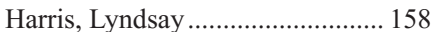

Harrison, Beth ...................... 275, 1135

Harrison, Grant ………………....... 688

Harrison, Jenna ............................... 1514,15

Harruff, Richard C .......................... 10

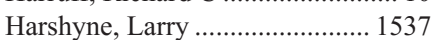

Hart, Jesse ......................... 1230, 1983

Hart, John .......................... 632, 1632

Hart, Phil A....................................... 634

Hartley, Christopher .... 170, 396, 572, $644,689,744,770,1617,1830$

Hartman, Aaron ............................... 841

Hartman, Anne-Renee .................. 1214

Hartman, Douglas J......... 1543, 1557,

1560,1575

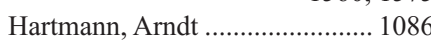

Hartmann, Karin ......................... 1371

Hartzband, Pamela .............. 466, 2014

Harutyunyan, Ashot ......................... 54

Harwalkar, Jyoti .................................. 1800

Has, Phinnara ................................ 1835

Hasanovic, Adnan ....................... 1216

Hasegawa, Kiyoshi .......... 1660, 1661

Hasegawa, Mizue .......................... 1869

Hashisako, Mikiko ...................... 1867

Hassan, Anjum .......... 357, 1330, 1413

Hassan, Muhannad ................ 362, 397

Hassan, Oudai ........... 890, 944, 1217

Hasserjian, Robert P........ 1387, 1427, $1462,1473,1508,1512,1516$

1584

Hasteh, Farnaz........... 121, 478, 2009 ,

Hatakeyama, Kinta..... 2022

Hatanaka, Yutaka ......................... 1760

Hatch, Ellen........................................ 1371

Hatem, Joseph ................................. 1136

Hattori, Ryohei......... 405, 1063, 1064

Hau, Andrew M.......................... 1800

Haudenschild, Christian............... 1792

Have, Cherry L.................................. 900

Haverkos, Bradley.......................... 1335

Havrilesky, Laura J ...................... 1121

Hawkins, Doug ................................. 85

Hawley, Sarah J............................... 993

Hayashi, Akimasa ................1660.......1661

Haydu, Lauren E .......................... 2064

Hayek, Kinda .................................. 398

Hayes, Malcolm M.......................... 281

Hayes, Miriam...

658
Haynes, Allan................................ 1068

He, Hong-Lin ................................. 690

He, Jian ............................................. 171

He, Jianguo ...................................... 326

$\mathrm{He}, \mathrm{Jie}$........................................... 1505

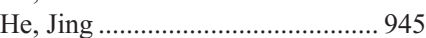

He, Mai ……………………......... 1835

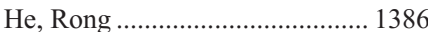

He, Xin ...................................................... 691

Heagley, Dawn ................................. 430

Healey, John ...................................... 34

Heaphy, Christopher M.... 1753, 1754

Heayn, Michelle ................. 1555, 1749

Hecht, Jonathan H.............1118, 1137

Hechtman, Jaclyn........ 718, 719, 795, 1801,1814

Hechtman, Jaclyn F....................... 785

Hedley, David ................................. 1774

Heffron, Cynthia ................. 524, 1265

Heher, Yael K ...................... 1958, 1959

Heider, Amer .................................. 467

Heimann, Alan .............................. 1947

Heintzelman, Rebecca................... 103

Heirich, Kyra................................... 456

Helba, Brian ..................................... 565

Helbig, Petra ………………….... 1810

Helenowski, Irene B....................... 203

Helgeland, Lars ........................... 1908

Helle, Deborah ............................. 1698

Hellmann, Matthew D................ 1910

Helm, Klaus F .................................. 505

Helm, Samantha.................................... 2046

Helman, Elena.................. 1792, 2052

Hemamali, Samaratunga ............... 946

Hembrough, Todd ........................ 1813

Hemmerich, Amanda ...................... 688

Hemminger, Jessica ..................... 1620

Hempel, Nadine ............................ 1246

Henderson, Ying C .......................... 603, 612

Hendrickson, Shelby ...................... 105

Hennessey, James V ............ 466, 2014

Hennrick, Kenneth ............................ 308

Henriksen, Jonathan C ........ 266, 560,

1997

Henriksen, Kammi J.............. 22, 1600

Henzler, Christine .......................... 182

Heo, Dae Seog ............................... 1407

Herculiani, Amanda P ......... 866, 867

Herman, James G ........................... 812

Hernandez Losa, Javier.................... 49

Hernandez-Cruz, Blandina............ 850

Hernandez-Losa, Javier................ 2073

Hernandez-Prera, Juan C .............. 554, $1279,1294,1295$

Hernandez-Toriz, Narciso ............. 850

Hernandez, Marier .......................... 502

Hernandez, Mike......................... 1022

Hernandez, Monica .......................... 1577

Hernandez, Silvia ........ 893, 947, 948 ,

960,2055

Hernandez, Valeria …..................... 543

Herrera Hernandez, Loren P ......... 999

Herrera, Guillermo A ...... 1587, 1597,

1623,1981

Herrera, Hannah B ......................... 608

Herrero, Laura..................... 630, 2042

Hes, Ondrej ... 873, 1010, 1027, 1062 ,

1072,1074

Heuer, Monica.................................... 548

Hewer, Ekkehard........................... 1972

Hexner, Elizabeth......................... 1486 
Hickey, Tyler. Hickman, Richard ....................... 949 Hicks, David G.... 138, 205, 295, 296,

Hicks, Jessica ............. 849, 930, 1703

Hicks, John................................. 2043

Hida, Tomoyuki ............... 1866, 1868

Higgins, John P ......................... 1973

Higgins, Sara.................................... 692

Highfield Nickols, Hilary .................. 7

Hijazi, Nouf........................................ 1437

Hill, Charles E................................. 559

Hill, D Ashley .................................... 11

Hill, Dana A ……………………..... 980

Hillen, Lisa................................... 1387

Hillen, Uwe ................................ 1721

Hillier, Sharon ................................. 437

Hinoue, Toshinori............................ 846

Hinterbrandner, Magdalena......... 1482

Hinton, Alice .................................. 1591

Hipp, Jennifer................................ 399

Hirabayashi, Kenichi........ 1738, 1772

Hirano, Michio ............................... 2079

Hirano, Satoshi............................. 1760

Hirata, Masahiro ......................... 1406

Hirokawa, Mitsuyoshi................... 597

Hirose, Ryutaro ............................... 1755

Hirose, Takanori............................ 1656

Hiroshima, Kenzo ............. 1869, 1935

Hirsch, Michelle.......... 932, 933, 990,

1074,1118

Hirschowitz, Lynn........................ 1229

Hissong, Erika.................... 693, 1138

Hlady, Ryan A ................................ 647

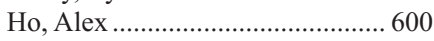

Ho, Caleb .......................................... 57

Ho, Cheng-Ying ................................. 11

Ho, Cheryl..................................... 1919

Ho, Donald M-T............................ 1704

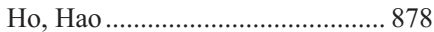

Ho, Jonhan ............... 510, 1557, 1582

Ho, Ye-Shih....................................... 903

Hoadley, Katherine A.................... 846

Hoang, Lien... 1139, 1140, 1153, 1236

Hoang, Mai P ................................ 502

Hobday, Timothy J ........................... 803

Hoch, Benjamin ................... 88, 1671

Hoda, Rana S ..................... 347, 1944

Hoda, Raza S................................... 468

Hoda, Syed ........................ 137, 1947

Hodara, Elisabeth ............................. 878

Hodgkinson, Kendra ..........1119, 1141

Hodgson, Shirley........................... 1048

Hodi, F Stephen ................ 1144, 1379

Hodi, Zsolt ...................................... 160

Hodjat, Parsa ......... 1374, 1388, 1389 ,

1390,1391

Hodul, Pamela J ........................... 1744

Hoehn, Daniela ................. 1992, 1993

Hoepner, Sabine ............................ 1482

Hoff, Kirsten E.............................. 2079

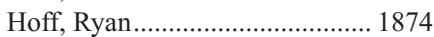

Hoffman, Ronald........................... 1584

Hoffmann, Jenny ............... 1392, 1973

Hofstetter, Wayne L ........................ 623

Hogan, Kevin ........................ 400, 950

Hogeboom, Adriana .......................... 1706

Hohenwalter, Mark ...................... 1565

Hojilla, Carlo................................. 1393

Holdhoff, Matthias ........................ 1703

Holland, Eric C ........................... 1709
Holland, Steven M ........................ 1405

Hollebecque, Antoine................... 1822

Holleran, Evan ..................................58

Hollowell, Monica ........................... 458

Holman, Carol............................... 1381

Holmes, Margaret P .................... 1705

Holthoff, Emily .......... 907, 957, 1142

Holtzclaw Williams, Pamela......... 552

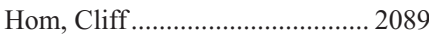

Homayoun, Farrah .......................... 913

Hon, Jane D...................................... 185

Hong, Christine ............................. 1974

Hong, Hong .................................... 1842

Hong, Seung-Mo.... 1750, 1753, 1754

Hong, Soon Won ................. 576, 2032

Hooper, DCraig ............................ 1537

Hooper, Jody E.................. 13, 25, 641

Hopkins, Benjamin ....................... 1816

Hoque, Mohamed............................ 856

Hora, Milan ....................... 1010, 1027

Horbinski, Craig.......................... 1722

Horn, Paul .................................... 1719

Horn, Susanne ................................ 1721

Horn, Thomas................................... 519

Hornick, Jason.................... 1048, 1745

Hornick, Jason L ... 55, 59, 67, 89, 94 , 294, 581, 736, 1544, 1775, 1950

Horning, Lisa .............................. 2000

Horowitz, Neil.................. 1144, 1248

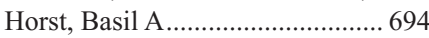

Horst, David ....................................... 694

Horvai, Andrew E ........ 51, 657, 1224

Horvath, Bela ......... 1662, 1663, 1664

Horwitz, Steven M ....................... 1375

Hoshi, Masato .............................. 1604

Hoskin, Tanya L ................... 119, 204

Hosking, Paul......... 1333, 1446, 1447

Hossain, Maheen.......................... 1143

Hosseini, M .................................... 820

Hosseini, Mojgan ........................ 2078

Hossler, Carrie .............................. 1246

Hotchandani, Nihar ........................ 468

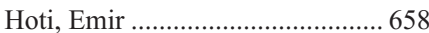

Hou, Yanjun .................. 172, 210, 401

Hou, Yingyong ............................. 1885

Houghton, Donald C ........................... 5

Hourseau, Muriel .......................... 1301

Houser, Kenneth R ....................... 1246

Houser, Patricia M ........................ 474

Houser, Steven R............................ 315

Hovelson, Daniel H........... 1278, 1805

Howe, James R................................ 568

Howe, Philip H............................. 1800

Howitt, Brooke E .......599, 990, 1118,

$1120,1144,1180,1200,1234,1239$

1248,1252

Howlett, Christopher..................... 953

Hoyle, Aoisha.................................... 1100

Hoyle, Eric .................................... 1394

Hoyt, Clifford C ................. 2039, 2057

Hrebinko, Ronald L......................... 355

Hristov, Alexandra ......................... 487

Hruban, Ralph H... 1738, 1753, 1754,

1772,1775

Hsi, Andy C....................................... 501

Hsi, Eric D ............ 1372, 1445, 1473,

$1479,1480,1486,1508,1511,1522$

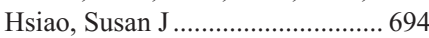

Hsieh, Eugene ................................ 1098

Hsu, Amy P ................................... 1405

Hsu, Chih-Yi ...... 134, 395, 412, 1704
Hsu, Peihong.

1380

Hsueh, Chuen........................ 173, 297

Hu, Buqu ............................................ 761

$\mathrm{Hu}$, Shaomin ................................... 174

Hu, Shimin ............ 1347, 1352, 1377,

1385, 1395, 1396, 1416, 1419, 1450,

$1492,1509,1521$

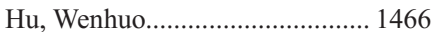

$\mathrm{Hu}$, Yan.......................................... 1280

Hu, Zhihong ..................... 1395, 1396

Huang, A ....................................... 820

Huang, Dan .......................... 751, 805

Huang, Gang ............................... 1616

Huang, He ....................................... 951

Huang, Hsien-Neng....................... 1168

Huang, Hsuan-Ying....... 75, 76, 1168 ,

1671

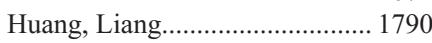

Huang, Qin.................................. 1421

Huang, Shian................................... 326

Huang, Shih-Chiang...... 58, 65, 1304,

1836

Huang, Weei-Yuan ........................ 800

Huang, Weei-Yuarn ............. 732, 1921

Huang, Wei................................... 618

Huang, Weihua...69, 854, 1159, 1160,

1539,1571

Huang, Xin.................................... 1352

Huck, Amelia ............................... 1974

Hudis, Clifford ................................. 201

Hudson, Chad......... 1397, 1398, 1452

Huebner, Thomas .............................. 695

Huggins-Puhalla, Shannon............ 288

Hughes, Ian ................................... 1071

Huguenin, Anne-Laure................. 1482

Huh, Gi Young ..................... 627, 760

Huh, Jooryung ................................ 1015

Huh, Yang O................................. 1416

Huho, Albert..................................... 1399

Hui, Ling ........................................ 175

Hui, Pei ............................. 1026, 1232

Hui, Yiang .......696, 1069, 1145, 1146,

1547,1793

Humayun, Irshad........................... 116

Hummelen, Paul Van.................... 1696

Humphrey, Peter A...... 841, 915, 952,

1026

Humphreys, Elizabeth................... 906

Hung, Yin.................... 59, 402, 1870

Hunninghake, Gary M ............... 1870

Hunt, Bryan............................ 396, 572

Hunt, Kelly K..... 106, 150, 307, 1457

Hunter, Robert L ...... 289, 1428, 1534

Huntsman, David G .........1131, 1132, $1139,1140,1153,1236$

Huo, Lei ................................. 149, 209

Hurtado-Coll, Antonio .................. 993

Husain, Aliya N....... 335, 1850, 1925,

Husain, Arjumand .........................1111

Husain, Sanam ................................. 1946

Hussaini, Mohammad O ............ 1400,

1528

Hussein, Shafinaz.......................... 1401

Hussey, George S .............................. 1800

Hussong, Jerry W ............... 1471, 1979

Huston, Hunter ................................ 1607

Hutchens, Kelli A................ 520, 1987

Hutchinson, Lloyd....... 509, 885, 982 ,

1523

Huynh, Tiffany G ............. 1757, 1871
Hwang, Chung Su ................ 627, 760

Hwang, David ............................... 403

Hwang, David M........................... 1863

Hwang, Michael.................... 915, 952

Hwang, Samuel ................................. 14

Hwang, Sangheum ........................ 2048

Hwang, Shen-An.......................... 1534

Hwang, Soohyun........................... 2048

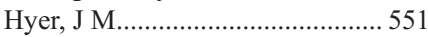

Hyjek, Elizabeth............................. 1328

Hyland, John ................................. 680

Hyrcza, Martin .................. 1282, 1316

Hyrcza, Martin D .......................... 1281

Iacobuzio-Donahue, Christine ... 1801

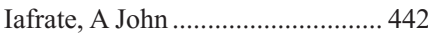

Iafrate, Anthony J .......................... 320

Ibarra, Javier ...................................... 1197

Ibrahim, Ashley ................................... 404

Ibrahim, Gena ....................953, 1610

Ibrahim, Mohamad........................... 513

Ibrahimpasic, Tihana......................... 614

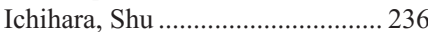

Ichino, Martina............................... 1461

Iczkowski, Kenneth A .......... 943, 954, $965,1073,1565$

Ide, Hiroki ............................. 961, 963

Idoate, Miguel A.................. 421, 1706

Idowu, Michael O ............ 1990, 2004

Idrees, Mohammed .............. 941, 942

Idrees, Muhammad T .......... 920, 921,

1020,1031

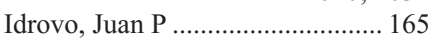

Ieremia, Eleni ................................... 512

Iglesias, Alejandra ............................ 1813

Iglesias, Alejandro D................... 2079

Iglesias, Carmela........................... 2073

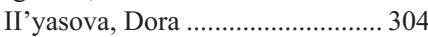

Ijiri, Rieko ........................ 1845, 1846

Ikegami, Masahiro ........................ 1037

Ikpatt, Offiong F............... 1370, 1437

Ikpatt, Offiong Francis ............... 1490

Illei, Peter B ................................... 1854

Imber, Michael J ............................. 489

Inagaki, Akiko................................. 268

Inamdar, Kedar V ........................... 1369

Inamura, Kentaro ............................... 1915

Incharoen, Pimpin ......................... 1891

Inderbitzin, Daniel .................................. 662

Ingram, Davis............................. 53, 61

Inomoto, Chie ................................ 598

Inoue, Satoshi.................................. 961, 963

Inoue, Tomohiro ...............................597

Inwards, Carrie Y ......................... 43, 1290,

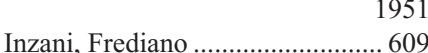

Ionescu, Diana N....................................... 1919

Iorgulescu, Bryan ......................... 1707

Ioris, Rafael.................................. 1250

Iossifov, Ivan............................... 1828

Iowa NET SPORE ……………......... 568

Ip, Philip..................................... 1147

Iqbal, Jabed ................... 104, 176, 177

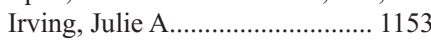

Isaacs, John .................................. 931

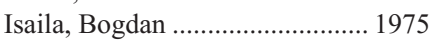

Ishida, Hirotaka ................................ 583

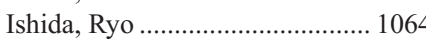

Ishikawa, Nahoko ........................... 735

Ishikawa, Noriyoshi ...................... 735

Ishikawa, Yuichi......... 93, 1888, 1915

Ishizaki, Tatsuro ............................. 628 
Islam, Humayun ................................ 854

Islam, Shahidul .......................... 1120

Ismail, Mamudo R ....................... 1535

Ismail, Wesam .................................. 1611

Ismiil, Nadia........... 1087, 1097, 1098

Itami, Hiroe ……………………..... 236

Itani, Doha........................................ 80

Itani, Malak ........................................ 374

Itano, Osamu ................................... 1673

Ito, Masaaki.................................. 739

Ito, Noriko.......................................... 236

Itoh, Tomoo ……………... 1656, 1746

Iuga, Alina.............................. 695, 780

Ivan, Doina............ 488, 490, 523, 535

Ivelja, Sinisa................................... 719

Iwamoto, Sanju ................................. 1406

Iwasaki, Akinori ............................ 1866

Iwata, Hidehiro .................................. 405

Iyer, Gopa......... 847, 939, 1000, 1080

Iyer, Saipriya ................................. 276

Izawa, Jonathan.............................. 916

Izevbaye, Iyare _...............................59 59

Jabado, Nada ........................................ 54

Jabbour, Mark ………………….... 178

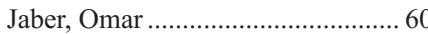

Jackson-Cook, Colleen ..................... 9

Jackson, Christopher R .................. 448

Jackson, Cynthia ................. 928, 1983

Jacob, Reuben P ......................... 1283

Jacobsohn, Kenneth ..................... 1565

Jacobson, Rebecca ....... 188, 189, 190

Jacquemont, Celine ....................... 1919

Jaffe, Elaine S .................. 1376, 1483

Jaffee, Elizabeth M ........................ 703

Jaffer, Shabnam.... 179, 180, 237, 267

Jagdale, Deepa ............ 71, 508, 1409,

1804,2031

Jagdis, Amanda ........................... 1281

Jain, Dhanpat...... 761, 813, 815, 1659

Jain, Nitin .................................... 1424

Jain, Richa........................................ 169

Jain, Sanjay ...................... 1601, 1604

Jain, Sarika ................................... 1402

Jain, Shilpa .................................. 1167

Jajoo, Kunal ......................................... 801

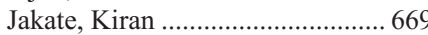

Jakubowski, Maureen A................ 486

Jalali, Rakesh ..................................... 1701

Jamal, Mohsin ...................... 955, 956

Jamali, Faek ................................... 178

James, Aaron ....................................... 79

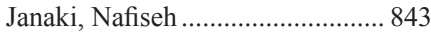

Janakiram, Murali ........................... 222

Janco, Jo Marie ............................ 1225

Jang, Erika L ................................... 321

Jang, Jin-Young ................. 1738, 1772

Jang, Kee-Taek...... 1738, 1743, 1763,

1765,1772

Jang, Kiseok ............... 101, 706, 1691

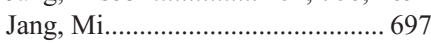

Jankowitz, Rachel ........................... 166

Janne, Pasi A .................................... 1909

Janowczyk, Andrew .......... 269, 1561,

1562,1583

Janssen, Emiel AM........................ 2059

Jarboe, Elke...................................... 115

Jardin, Fabrice..........................38, 1809

Jaso, Jesse M.................... 1428, 1465

Javadi, Mehrbod S ........................ 927

Javidiparsijani, Sara ....................... 594

Javier, Breanna M

180
Jawale, Rahul M............................ 1802

Jazaeri, Amir A.............................. 1158

Jean-Gilles, Jerome ……………..... 857

Jedrzkiewicz, Jolanta ............... 61, 698

Jeffrey, Stefanie S ............................456

Jeffreys, Matt .................................. 957

Jehi, Lara...................................... 1702

Jelloul, Fatima-Zahra ....... 1380, 1563

Jen, Kuang-Yu................... 1593, 1651

Jencks, Amy ....................................... 398

Jeney, Csaba.................................... 1251

Jenkins, David............ 870, 909, 1035

Jenkins, Robert B .......................... 1725

Jenkins, Sarah M........... 4, 314, 1254, $1849,1893,1911,2093$

Jenkins, Stephen........................... 1607

Jenkins, Taylor M............................. 699

Jennings, Timothy A....................... 1260

Jensen, Kristin C .................... 114, 294

Jensen, Thom .............................. 2044

Jensen, Tyler A ............ 205, 339, 2044

Jentoft, Mark E............................. 1724

Jeon, Yoon K …………………….... 1877

Jeon, Yoon Kyung .............. 1407, 1876

Jeong, Se Un ..................................... 459

Jessup, Morgan............................. 1560

Jessurun, Jose.............. 659, 817, 1901

Jeung, Jennifer ............................. 1662

Jevremovic, Dragan ..................... 1442

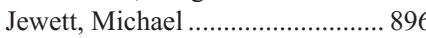

Jhala, Nirag ….............. 318, 337, 468

Jhala, Nirag C................................. 331

Jhavar, Sameer ............................... 1277

Ji, Yuan.

1419,1521

Jia, Liwei.

958,1708

Jiang, Hui .... 988

Jiang, Xiangnan............................. 1403

Jiang, Yi ............................1171, 1172

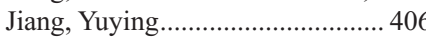

Jiang, Zhong.................................. 982

Jimenez, Jose.................................. 1813

Jimenez, Rafael E....... 959, 999, 1884

Jin, Gang .......................................... 1782

Jin, Ming ............................. 407, 793

Jin, Mulan .................................... 1249

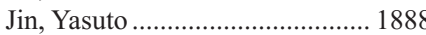

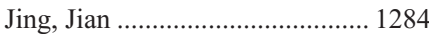

Jing, Xin ................................ 422, 467

Jo, Vickie Y.......... 62, 428, 458, 1285

Jobe, Blair A...................................... 1933

Jochumsen, Kirsten M .................1118

Joehlin-Price, Amy S .......... 181, 1148

Johannsen, Shulammite.................. 946

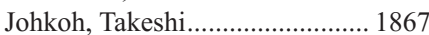

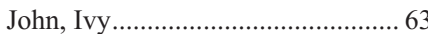

Johncilla, Melanie ............................ 700

Johns, Cameila …............................ 1675

Johnson, Adrienne ............... 271, 1927

Johnson, Arthur .............................. 182

Johnson, Bruce E ………………..... 1900

Johnson, Charnell........................... 1994

Johnson, Cory …………………... 1384

Johnson, Daniel N................ 575, 582

Johnson, Geoffrey B ................... 1911

Johnson, Howard............................. 2012

Johnson, Kevin C ......................... 1802

Johnson, Michael H .............................. 906

Johnson, Rebecca L...........................558

Johnson, Timothy M …………..... 1805

Johnston, Abbey ............................ 1219

Jolly, Grant.

1728
Jones, Andrea ................... 1665, 1666

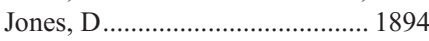

Jones, Dan ...................................... 1529

Jones, Daniel ................................... 1829

Jones, David M ............ 528, 629, 716

Jones, Katie N …………...... 229, 305

Jones, Kirk ……………………......... 365

Jones, Martin.................................. 442

Jones, Mildred.................................. 304

Jones, Mirka W ………………..... 1238

Jones, Ryan ......................................... 701

Jones, Tara...................................... 1541

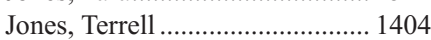

Jonsdottir, Kristin ........................ 2059

Jorda, Merce........ 375, 387, 416, 602, $943,969,1073$

Jordan, Emmet J.................. 847, 1080

Jordan, Sheryl ................................ 120

Jordan, Suzanne ............................ 1152

Jordao, Dercio .................................. 1535

Jorgensen, Jeffrey L .................... 1424

Jorns, Julie............ 108, 131, 183, 991

Joseph, Nancy ....... 51, 213, 214, 869,

1149,1233

Joseph, Nancy M......365, 1165, 1175,

1189

Joshi, Amit .

... 995

Joshua, Anthony ........................... 2, 317

Josselin, Nicolas............................. 493

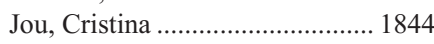

Joubert, Philippe ............................... 336

Jour, George .................................. 54, 64

Ju, Jennifer ................................. 1700

Juanpere, Nuria ... 893, 947, 948, 960, 2024, 2045, 2055, 2077

Judd, Andrew ................................. 455

Julian, Jeffrey ................................. 1728

Jun, Dae Won ................................ 1691

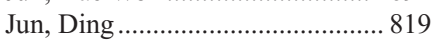

Jun, Sun-Young ............................ 1750

Jung, Soo Jin .................................. 1015

Jungbluth, Achim .............. 1382, 1475

Juric-Sekhar, Gordana................. 1709

Juskevicius, Ridas .................................. 1491

Juskewitch, Justin ............................. 15

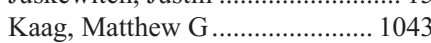

Kaczmarowski, Amy .................... 1565

Kada, Akiko ................................... 236

Kadi, Wendy ................................. 1872

Kadokura, Alexander ..................... 502

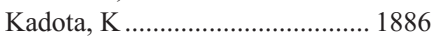

Kadri, Sabah................................... 710

Kaffenberger, Benjamin ................. 495

Kahl, Brad ..................................... 1451

Kajdacsy-Balla, Andre ........ 215, 793,

Kajiwara, Hiroshi ............................ 598

Kakar, S............................. 810, 820

Kakar, Sanjay ... 657, 727, 1634, 1678

Kakar, Sham S........................... 1322

Kalari, Krishna R .......... 229, 230, 305

Kalariya, Jigna .............................. 1048

Kalhor, Neda ........ 1873, 1874, 1906, $1931,1936,1937$

Kalife, Elizabeth ................. 184, 1150

Kalir, Tamara L ............................... 454

Kallakury, Bhaskar VS.................... 1082

Kallen, Michael E ........................ 1405

Kalloger, Steve ............................. 1751

Kalloger, Steve E .......................... 794

Kalusova, Kristyna............ 1010, 1027
Kamal, Mohammed...................... 1088

Kamat, Ashish .............................. 935

Kambham, Neeraja ...................... 1973

Kamel-Reid, Suzanne .................. 1393

Kaminski, Mark ........................... 2008

Kamiza, Steve ………………....... 1362

Kampani, Coxcilly ...................... 1362

Kamran, Waseem .......................... 1191

Kanaan, Yasmine............................... 187

Kanagal-Shamana, Rashmi ....... 1374, 1388, 1389, 1390, 1391

Kanagal-Shamanna, Rashmi ..... 1422,

1464

Kanagasabapathy, D Anand Rajan ...

1286

Kanber, Yonca ........................ 362, 397

Kanda, Hiroaki .................................. 93

Kandukuri, Shivani ........................... 408, 409

Kane, Shubhada ..... 1288, 1297, 1300

Kane, Yehonatan ............................. 185

Kang, Gyeong Hoon ...................... 753

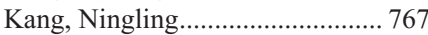

Kang, So Young ................................... 1714

Kang, Yuna..................................... 1151

Kannangai, Rajesh ................................. 686

Kanno, Atsushi ................................ 1769

Kansal, Rina ...................................... 1472

Kantarjian, Hagop ........................ 1509

Kantekure, Kanchan....................... 831

Kantoff, Philip W ......................... 1065

Kao, Ken ....................................... 2013

Kao, Yu-Chien............................. 58, 65

Kaplan, Edwin L ........................... 575

Kapp, Meghan............................... 1603

Kapur, Payal.......................... 913, 914

Kapus, Andras .................................. 981

Kapusta, Linda R ........................ 1995

Karabakhtsian, Rouzan G ... 188, 189,

190, 394

Karagiannis, George S ................. 675

Karam, Jose A .................................. 1044

Karamchandani, Dipti M ............ 1968

Karamitopoulou, Eva ....... 1752, 1780

Karandikar, Nitin ............................ 1381

Karasinska, Joanna....................... 1751

Karbelashvili, Mirian ...................... 2052

Karia, Sushil....................................... 1048

Karlon, William................................ 431

Karnes, R Jeffrey............................ 999

Karnezis, Anthony.............1152, 1153

Karumanchi, Ananth ..................... 1137

Kasai, Kazunari...............................597

Kasajima, Atsuko ............................. 583

Kashgarian, Michael ................... 1628

Kashikar, Nilesh D......................... 778

Kashiwagi, Eiji...................... 961, 963

Kaspirkova, Jana ............................ 1154

Kassouf, Wassim .............................. 1070

Katabi, Nora.......... 1318, 1319, 1320,

132

Katerji, Hani................................ 1051, 1398

Kates, Max ....................................... 856

Kato, Masashi ................. 1063, 1064

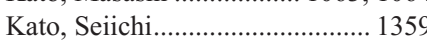

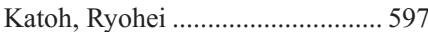

Katz, Matthew H.............................. 726, 750

Katzenberg, Jennifer .................... 962

Kauffman, Lauren R .................... 1351

Kaufman, Elizabeth........................ 878

Kaushal, Rajiv

1297 
Kawa, Shigeyuki ............... 1768, 1769

Kawabata, Ayako ......................... 1155

Kawahara, Kunimitsu ................. 1868

Kawahara, Takashi ................ 961, 963

Kawahara, William ....................... 381

Kawai, Tatsuo............................... 1585

Kawasaki, Tomonori ..................... 236

Kazemimood, Rossana.................. 702

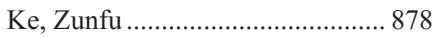

Keam, Bhumsuk......................... 1877

Keaney, Debra...

Kearney, Padraig .........

Keefe, Daniel T

1243

... 862, 950

Keegan, Helen .................... 1251, 1317

Keeney, Gary L ...... 1156, 1951, 2005

Keeney, Michael .......................... 1156

Kehr, Elizabeth............................. 1157

Keibler, Mark A.............................. 1332

Keller, Charles .............................. 1830

Kelley, Alyssa ............................... 1379

Kelley, Todd W ................. 1503, 1508

Kellough, David........................... 1529

Kelly, Alyssa .................................. 1144

Kelly, Brian ......................... 118, 1574

Kelly, Kevin .................................. 2046

Kelly, Lori A.................................. 1933

Kelly, Lynne ...................... 1243, 1251

Kelly, Melissa ………………….... 558

Kelly, Paul........................ 796, 1777

Kelly, Ronan ...................................... 806

Kelly, Thomas J............................ 1142

Kelting, Sarah M.......................... 1158

Kendall, Monica L ....................... 1951

Kennedy, Erin .................................. 675

Kennedy, Susan...... 1317, 1717, 1718

Kennelly, Rory ............................... 680

Kensler, Kevin ............................... 117

Keohane, Catherine.......................... 18

Kernag, Casey ......... 2026, 2051, 2085

Kerr, Darcy A .......................... 45, 610

Kerr, Sarah E........... 446, 1225, 1254,

1951

Kesterson, Joshua P.......... 1127, 1246

Ketwaroo, Gyanprakash................ 748

Kevil, Chris ................................... 1344

Keyashian, Kian............................... 641

Keyoumarsi, Farah...................... 1439

Kezlarian, Brie ............. 107, 186, 964

Khachaturov, Vadim... 66, 67, 68, 506

Khaira, Depinder..

... 241

Khalifa, Mahmoud ........... 1087, 1097, $1098,1122,1924,2001$

Khalifeh, Ibrahim .............. 1259, 1276

Khalighi, Mazdak........................ 1607

Khalil, Farah ………………….... 1022

Khalil, Kamal .................................... 313

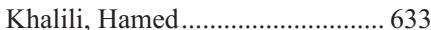

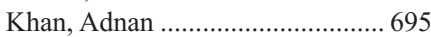

Khan, Ali ........................................ 2025

Khan, Faisal

.... 908

Khan, Farhan........................ 187, 235

Khanafshar, Elham................. 388, 595

Khani, Francesca..... 994, 1024, 1029, $1055,1159,1160,1998,2018$

Khanlari, Mahsa ................................ 301

Khanna, Abha.................................. 371

Khararjian, Armen............... 1875, 1976

Khatri, Sameer .................................. 503

Khattar, Pallavi............69, 1159, 1160

1539

Khaw, Y-Ling 680
Khaw, Yi Ling ............................. 1977

Khella, Heba WZ ……………..... 1034

Kherad Pezhouh, Maryam ........... 681, 703,771

Khoury, Joseph D.... 425, 1457, 1464 1492,1509

Khoury, Thaer ..... 188, 189, 190, 191, 192,193

Khurana, Kamal K ....... 432, 433, 434 Khurram, Nigar ................................ 704

Kiehl, Rasmus ...................................... 3

Kiernan, Colleen M............................. 469

Kilari, Deepak .................................. 965

Kilgore, Mark R ............................. 266

Kilic, Fusun.................................. 1096

Killian, Jonathan ........................... 1803

Kim, Ahrong ………............ 627, 760

Kim, Byeong Gwan ..................... 1650

Kim, Chae Hwa................. 1370, 1495

Kim, Charlie................................. 1549

Kim, Christopher J ....................... 1564

Kim, Chul Whan ................. 707, 1015

Kim, Chul Woo …………………... 1407

Kim, Daniel....................... 2051, 2085

Kim, Dong-Hoon ............................... 194

Kim, Eric S...................................... 965

Kim, Eun Kyung ............................. 705

Kim, Grace....................... 1161, 1766

Kim, Grace E ............ 772, 826, 1755 ,

1761,1767

Kim, Haeryoung............................ 1753

Kim, Hannah .................................... 1407

Kim, Hoguen .......................... 697, 705

Kim, Hyo-Eun................................ 2048

Kim, Hyun-Jung............................ 1015

Kim, Hyunseok ................................. 205

Kim, Hyunsung ....................................... 706

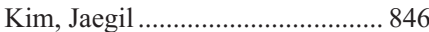

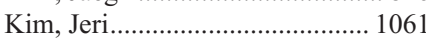

Kim, Jhingook …………………. 1858

Kim, Ji Eun ..................................... 522

Kim, Ji Yeon.................................. 1015

Kim, Ji-seop .................................... 576

Kim, Jinah ......................................... 505

Kim, Jiyoon................................... 1753

Kim, Jong T......................... 504, 2047

Kim, Joo Young.................... 707, 1754

Kim, Jung.................................... 1014

Kim, Kyoung................................... 796

Kim, Kyoung-Mee ................ 720, 827

Kim, Kyu-Rae ....................1162, 1163

Kim, Min J ................................. 1742

Kim, Moon-Young ........................ 1876

Kim, Robert..................................... 23

Kim, Sang Kyum ............................... 1015

Kim, Sehui ........................ 1407, 1876

Kim, Seokhwi .............................. 2048

Kim, Seong-Ik............................. 1710

Kim, Seungwon............................. 1310

Kim, Sol-Min ...................................... 787

Kim, Sungeun ........... 271, 1287, 1860

Kim, Sunhee S ............................ 1755

Kim, Tae Min ................................. 1407

Kim, Tae-Beom ............................ 1044

Kim, William Y ................................. 846

Kim, Won ..................................... 1650

Kim, Young Geum ................ 627, 760

Kim, Young S ......... 1329, 1423, 1450

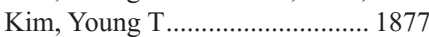

Kim, Yujin ..................................... 1710

Kimbrell, Hillary .............................. 505
Kimura, Kyosuke

1064

Kimura, Takahiro .......................... 1037

King, Caleb .................................. 1990

King, Rebecca .................... 1408, 1426

King, Tari ....................................... 154

Kini, Ameet ...................................... 1487

Kini, Usha ..................................... 1831

Kinlaw, Kathy .................................. 559

Kinloch, Mary A............................. 1139

Kinoshita, Yoshikazu ..................... 735

Kinsey, Richard S........................... 743

Kinzler, Kenneth ....... 886, 887, 1004,

1053

Kip, Nefize ……….............650, 1960

Kipp, Benjamin R ........... 1685, 1724

1855

Kircheva, Diana ............................ 1850

Kirimlioglu, Hale .......................... 1626

Kirk, Scott ...................................... 2052

Kirkwood, Kimberly S................. 1755

Kirsch, Richard ................... 675, 1540

Kiss, Katalin.................................. 1269

Kitagawa, Norihiko ........................ 1845

Kitagawa, Yuko ............................. 1673

Kiyokawa, Takako .............................. 1155

Klapman, Jason ............................. 1744

Klein, Eric A......................... 905, 989

Klein, Melissa ................................ 360

Klein, Molly .................................. 182

Klein, Robert R .........348, 1110, 1969

Klein, Roger D ............. 151, 152, 486

Kleinman, George ........................... 781

Kleinschmidt-DeMasters,

Bette K

1839

Klersy, Catherine.............................. 609

Kliethermes, Stephanie ...... 520, 1987

Klijanienko, Jerzy .......................... 593

Klimek, Szczepan ............... 983, 1081

Klimov, Sergey...... 195, 196, 207, 304

Klimowic, Alexander C .............. 1281

Klimstra, David.............. 64, 693, 718, $719,785,795,1579,1738,1740$

1772,1775

Klinge, Petra M........................... 1728

Klingen, Tor Audun........................ 110

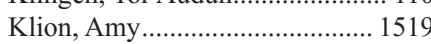

Kluge, Michelle L ......................... 324

Kluk, Michael J........ 347, 1817, 1828

Knapp, Michael................................. 14

Knechtges, Paul M...................... 1565

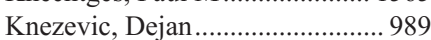

Knicely, Daphne............................ 1614

Knight, Jessica H ................................. 773

Knudsen, Beatrice ............. 1045, 2059

Knutsvik, Goril ............................ 110

Ko, Andrew H ............................... 1755

Ko, Huaibin Mabel......................... 837

Ko, Jennifer................................... 515

Ko, Jennifer S................. 67, 506, 507

Kobayashi, Kazuma ....................... 583

Kobel, Martin .......... 1111, 1139, 1140

1153

Kodira, Chinnappa ......................... 161

Koduru, Prasad...... 1345, 1346, 1355 ,

1460

Koehne de Gonzalez, Anne K.... 1667

Koelzer, Viktor ..................... 662, 663

Koeppen, Hartmut........................ 2067

Koepsell, Scott A................. 888, 1799

Koh, Jaemoon .............................. 1876

Koh, Valerie …...................... 291, 292

Kohashi, Kenichi...................... 70, 97

Kohler, Ted....................................316

Kohli, Manish ................................ 959

Kohlmann, Wendy K..................... 728

Kojima, Motohiro ………..... 739, 832

Kojima, Yumi ......................... 141, 197

Kokh, Dina .................... 410, 439, 470

Kokudo, Norihiro .............. 1660, 1661

Kolar, Grant .................................... 767

Kolhe, Ravindra .......... 71, 508, 1409, $1711,1804,2031$

Kolinsky, Michael ....................... 1001

Kollmeyer, Tom ........................... 1725

Kolomietz, Elena.................1115, 1116

Kolowitz, Brian J ......................... 1575

Kolquist, Kathryn A .......................... 505

Komatsu-Fujii, Takayoshi............. 735

Komorkji, Rami S ......................... 1432

Komrokji, Rami S ....................... 1444

Kondo, Jordan K ............................1114

Kondo, Tetsuo ………………….......597

Kondoh, Yasuhiro............................. 1867

Konety, Badrinath R..................... 1988

Kong, Beihua ................................ 1204

Kong, Beihuan .............................. 1205

Kong, Christina S........ 389, 413, 423,

449,450

Kong, Hui...................................... 1885

Kong, Max .................. 881, 949, 966

Konishi, Eiichi .................................. 72

Konno, Fumiko …………..... 198, 270

Konoplev, Sergej............................... 1385

Konopleva, Marina ……………... 1424

Konopleva, Sergej Naumovich .. 1424

Koo, Jamie …………………...... 1695

Kooby, David .......... 772, 1763, 1764,

1766,1767

Kopach, Pavel ...................... 708, 967

Kopetz, Scott........................ 642, 1104, 1788

Kos, Zuzana ......................... 281, 1120

Koshiol, Jill ........................ 1738, 1772

Kothari, Tarush.............................. 2015

Kotliar, Justin A................................ 584

Kott, Marylee M........................... 1428

Kotzer, Katrina E ............................ 324

Kouba, Erik .................................... 968

Koutlas, Ioannis ............................ 1261

Koutzaki, Sirma ….............................. 2088

Kovacs, Christina ................ 610, 1312

Kowalski, Diane..................... 341, 353

Kowalsky-Moskaliuk, Daniel ....... 361

Kozakewich, Harry ………………..... 36

Kraft, Adele O..................................... 2004

Kraft, Ruth

119,133

Kragel, Peter ......................... 549, 584

Kramer, Paula M ........................... 1625

Krane, Jeffrey F... 428, 431, 441, 458 ,

599,613

Krasinskas, Alyssa ............ 773, 1738 ,

$1748,1764,1765,1771$

Krasinskas, Alyssa M.......... 351, 393 , $636,772,826,1759,1766,1767$,

1772,1784

Krausz, Thomas ............... 1328, 1850

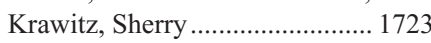

Krcedinac, Jelena ............................. 554

Kreisel, Friederike H......... 1330, 1413

Kresak, Jesse L................................. 647

Krinchai, Teppituk ........................ 1575

Krings, Gregor .... 121, 122, 213, 214 , 
Krishna, Murli................................ 1686

Krishna, Somashekar G ............. 2072

Krishnamurthy, Savitri........ 199, 444 ,

2049

Krishnamurti, Uma ..... 200, 207, 209, $238,242,243,351,393,1771$,

1964, 1965

Krishnan, Kumar............................ 427

Krishnan, Sunil ………………....... 726

Krizova, Adriana ......................... 1033

Kroft, Steven ........... 1333, 1446, 1447

Kroman, Eric............................... 1058

Krone, James T ................................1117

Kroneman, Trynda N ................... 803

Kroshinsky, Daniela ...................... 519

Krueger, Alex R ................ 1712, 2053

Krueger, JoEllen........................... 1460

Krysiak, Robert............................. 1362

Krystel-Whittemore, Melissa....... 201

Kryvenko, Oleksandr N ...... 610, 943, 969, 1047, 1073, 1312

Kshirsagar, Malti.......................... 1224

Kuan, Shih-Fan ..................... 673, 774

Kuang, Fei Li .............................. 1519

Kuba, Maria G ……....................... 155

Kubal, Chandrashekhar............... 1679

Kubo, Hajime ..................................... 315

Kucherlapati, Raju ......................... 846

Kudlow, Brian .............................. 1170

Kudose, Satoru .............................. 1604

Kuhn, Elisabetta ............................. 264

Kuk, Deborah................................... 795

Kulac, Ibrahim ............................. 970

Kulkarni, Rajan ............................... 456

Kulkarni, Shashikant.................... 1914

Kulkarni, Tanvee ........................... 1300

Kumar, Kirthi ...................... 1346, 1349

Kumar, Rajiv ..................... 1288, 1300

Kumar, Tripti................................ 1605

Kumchala, Prasanthi ............ 789, 790

Kummerlin, Intan.......................... 972

Kunchala, Sudhir.......................... 1043

Kunder, Komal............................ 1278

Kundisova, Alexandra.....................543

Kung, Vanderlene............................ 709

Kunju, L Priya ................................... 1074

Kunju, Lakshmi P ............. 987, 1039

Kunkalla, Kranthi.............. 1370, 1495

Kunz, Pamela L............................ 1781

Kuo, Frank ............... 752, 1144, 1462

Kuo, Kuan-Ting .............................. 1168

Kurani, Hetakshi .......................... 1701

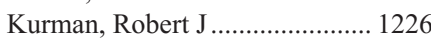

Kurnit, Katherine ........................ 1164

Kurtin, Paul J ... 163, 319, 1590, 1611

Kurtycz, Daniel FI ........................ 438

Kutys, Robert .................................. 322

Kvasnicka, Hans Michael .......... 1410

Kwak, Heewon............................... 710

Kweldam, Charlotte ............. 971, 972

Kwiatkowski, David J........ 846, 1900

Kwon, Deukwoo ................. 1370, 1495

Kwon, Dohee ..................... 1407, 1877

Kwon, Ghee Young ....................... 1015

Kwon, Ji Eun.................................. 1015

Kwong, Stanley .............................. 411

La Fortune, Kristin........................ 2050

La Fortune, Kristin A ..................... 404

La Rosa, Stefano ......... 585, 609, 1500

Labiano, Tania.................................. 421

Laboissiere, Renato S..................... 711
Lachance, Dan............................... 1725

LaChaud, Gregory............................. 79

Lacombe, Jeff.................................. 553

Lacroix-Triki, Magali..................... 254

Lacroix, Ludovic.......................... 1822

Lacy, Antonio ................................ 2042

Ladanyi, Marc....... 57, 64, 268, 1803, $1814,1823,1880,1910$

Lade, Stephen............................... 1368

Ladich, Elena ........................ 322, 323

Ladwig, Nicholas ......................... 1165

Lafaro, Rocco.................... 1851, 1852

Laga, Alvaro C .............................. 1544

Lagana, Stephen M ........... 712, 1667,

2079

Lagoo, Anand ............................... 1502

Laheru, Daniel................................. 812

Lai, Chi K................. 694, 1271, 1314

Lai, Chiung-Ru ..................... 395, 412

Lai, Jinping ………………….......... 767

Lai, Keith ...........................................68 685

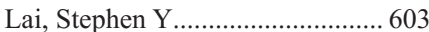

Lai, Win S ……………………..... 1059

Lai, Win Shun ...................................... 884

Lai, Yumei ........................................ 713

Laib, Annie.................................... 16

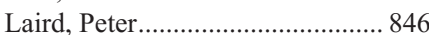

Lajer, Christel B ........................... 1269

Lakis, Nelli......................... 973,974

Lalla, Minesh .................................... 345

Lally, Kimberly ................................. 202

Lalonde, Amy........................................ 620

Lam-Himlin, Dora....... 634, 686, 799 ,

1657,1756

Lam, John.................................... 1498

Lambert, Jim R............................. 954

Lamon, Stefano ............................................ 84

Lamovec, Janez.................................111

Lamparski, Henry G......... 2026, 2051,

Lampreia, Leontino 2085

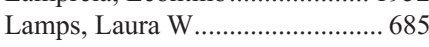

Lamzabi, Ihab …........................................ 169

Lan, Nan............................. 835, 1664

Lance, Raymond ............................ 993

Lancet, Jeffrey................................ 1501

Lanciault, Christian........................... 14

Landis, Charles ............................ 1646

Landolfi, Stefania ........................... 746

Landreneau, Rodney J.................. 1933

Lane, Jason..................................... 1318

Langer, Rupert ............. 663, 664, 670

Langford, Erin E .......................... 1981

Lanigan, Christopher P ........ 151, 152

Lantuejoul, Sylvie ......................... 1878

Laouirem, Samira............. 1672, 1676

Lapadat, Razvan..... 1945, 1975, 1991

Lapinski, James............................ 1662

Laquerriere, Annie ....................... 1809

Larque, Ana ........................ 1289, 1837

Larsen, Christian G ...................... 1269

Larsen, Christopher........... 1594, 1611

Larson, Alexandra .......................... 203

Larson, Benjamin ........................... 641

Larson, Brent...................... 1668, 1669

Larson, David................................... 514

Laskar, Derek ……………………..... 19

Laskar, Sarbani............................... 1288

Laskin, Janessa.............................. 128

Lasota, Jerzy ................................. 1862

Lastra, Ricardo R ............. 1166, 1200
Laszik, Laura.................... 1626, 2033

Laszik, Zoltan ........ 1593, 1626, 2033

Latario, Luke...................................509

Lau, Harold Y............................... 1281

Lau, Hubert D ............. 413, 449, 1412

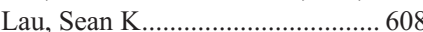

Laughlin, Todd S.............................. 303

Laureano, Julio..................................... 346

Lauria, Alexandra............................ 1168

Lauriola, Libero ……………….... 1721

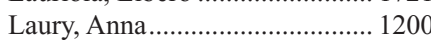

Lauwers, Gregory Y.... 627, 633, 667,

$672,792,796$

Laver, Thomas.................. 2026, 2051

LaViolette, Peter S ...................... 1565

Law, Charles ............ 565, 1556, 1599

Law, Jason.......................................... 976

Lawlor, Elizabeth R .......................... 91

Lawrence, H Jeffrey ........................ 989

Lawrence, W Dwayne..... 1088, 1145 , $1150,1196,1198$

Lawson, Barrett C ....................... 1167

Lawson, Diane ................... 1989, 2029

Lawton, Thomas............................ 120

Layfield, Lester ......... 476, 1879, 1978

Lazar, Alexander .................. 61, 2064

Lazaro, Conxi............................. 49, 83

Lazenby, Audrey J........................ 1799

Lazo de la Vega, Lorena... 1278, 1805

Lazzarini, Elisabetta.............. 329, 330

Le Bail, Brigitte .......................... 1638

Le Deley, Marie-Cecile .................. 1822

Le Guellec, Sophie.................... 73, 74

Le Stang, Nolwenn....................... 1878

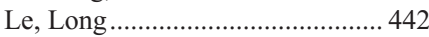

Leal, Conceicao............................... 258

Leandro, Gioacchino............................ 1041

Lebda, Paulette............................... 310

LeBrun, David P ............................... 1417

Lebwohl, Benjamin.......................... 712

Lech, Mazur ..................................... 702

Lechpammer, Mirna............ 586, 1712

Ledezma, Rodrigo A ...................... 845

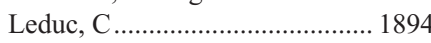

Leduc, Charles ... 314, 324, 325, 1880

Lee, Byron H...................... 847, 1080

Lee, Chang Hun .................... 627, 760

Lee, Cheng-Han ..... 1111, 1131, 1132 ,

$1139,1140,1153,1170$

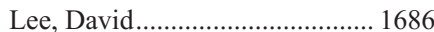

Lee, Eimear ........................ 1099, 1243

Lee, EunJee .................................... 1551

Lee, Eunjung .................................... 707

Lee, George ..................................... 1566

Lee, Hee Eun..... 204, 714, 715, 1644

1881

Lee, Hwa Jeong................................ 629

Lee, Hwajeong ............. 716, 717, 1680

Lee, Hyoun Wook ……………..... 1912

Lee, J Jack ................ 642, 1104, 1788

Lee, Jae Hoon................................ 1750

Lee, Jae-Hyuk ………..................... 1882

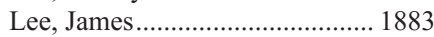

Lee, Jeffery E .................................. 726

Lee, Jeffrey E .................................... 750

Lee, Jen-Chieh ............................. 1168

Lee, Jeong Hyeon.............................. 707

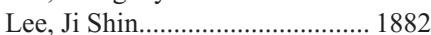

Lee, John ………………........ 1422

Lee, Jong Im.................................... 1015

Lee, Julieann

1015
Lee, Jungin ..................................... 2048

Lee, Kenneth R ………………......1118

Lee, KyoungBun ............... 1738, 1772

Lee, Kyung-Hwa............................ 1882

Lee, Larissa .................................. 1144

Lee, Lik Hang ......... 80, 718, 719, 785

Lee, Melody ................................. 2007

Lee, Mi-Sook ............................... 1858

Lee, Min Joung …………………..... 522

Lee, Minju........................... 720, 1714

Lee, Nathan ........................ 552, 1553

Lee, Paula S ................................ 1121

Lee, Peng..... 185, 966, 977, 978, 979 .

Lee, Sandra ...................... 1380

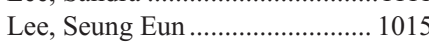

Lee, Seungjae...................................... 39

Lee, So Jeong ....................................... 627,760

Lee, Sun Mi..................................... 721

Lee, Wonae..................................... 1015

Lee, WonSok.... 71, 508, 1409, 1711,

1804,2031

Lee, Yi-Shan........................................ 1413

Lee, Ying-En ..................................... 690

Lee, Youngseok ...................................... 707

Lee, Zach........................................ 205

Leeman-Neill, Rebecca J ........... 1414 ,

$\begin{array}{ll} & 1415 \\ \text { Leen, Sarah L } & 1229\end{array}$

Lefebvre, Veronique....................... 1811

Lefevre, Marine................................... 593

Lefferts, Joel................................... 148

Lefterova, Martina ........... 1792, 2052

LeGallo, Robin.................................. 27

Legesse, Teklu....................... 439, 470

Lehman, John R ................................. 4

Lehrke, Heidi D ............................ 1756

Lei, Guang-Sheng .......................... 1943

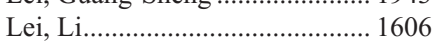

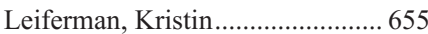

Leighton, Jonathan .............................. 799

Leise, Michael................................ 1658

Leite, Katia RM ................... 998, 1006

Lembo, Anthony ............................ 748

Leng, Bing............................ 105, 414

Leng, Nan....................................... 1792

Lenik, Joanna .................................... 543

Lennerz, Jochen K ……………..... 1757

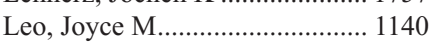

Leo, Patrick ................................... 1566

Leon del Castillo, Alicia ............... 677

Leon, Marino E ………………..... 1274

Leone, Dominick............................ 502, 516

Lepe, Marcos........................ 415, 975

Lepine, Charles ................................ 593

Lepor, Herber ................................. 983, 1081

Lerario, Antonop M ......................... 617

Lerner-Ellis, Jordan............................ 280

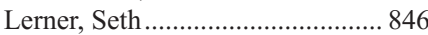

Leszczynska, Iga ………………….... 543

Leung, Sam …………………….... 1140

Leung, Samuel ...................1131, 1152

Levenson, Richard ............ 1712, 2053

Leventaki, Vasiliki ...................... 1497

Levi, Angelique W ....................341, 353

Levin, Mary...................................... 480

Levine, Douglas A............ 1235, 1236,

1250

Levine, Ross...................... 1475, 1514

Levinson, Howard............................. 513

Levy, David..................................... 389 
Lew, Madelyn ..................... 422, 467

Lewandowski, Raymond..................9

Lewin, David........................ 550, 551

Lewis, James ........... 1275, 1311, 1323

Lewis, Jean................................... 1290

Lewis, Jenna..................................... 416

Lewis, Julie .................................... 1384

Lewis, Natasha ................. 1169, 1468

Lewis, Susan …………………….... 822

Lewis, Suzanne ……………….... 1401

Lezcano, Cecilia...............................510

Li Ning Tapia, Elsa ...................... 1061

Li, Aihua .......................................... 976

Li, Anqi .......................................... 311

Li, Betty ........................... 1433, 1512

Li, Bill ........................................... 1989

Li, Chien-Feng ............. 75, 690, 1291

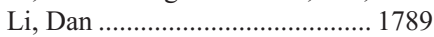

Li, Faqian ....... 606, 858, 1051, 1122,

1924

Li, Haonan....... 577, 666, 1532, 1635,

1636,1652

Li, Jianbo............................979, 1042

Li, Jianhong......... 881, 966, 977, 978 ,

Li, Jianyi ................................... 1806

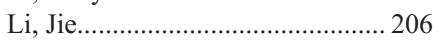

Li, Jieliang............................ 331, 337

Li, Jinhong ................................... 650

Li, K David ...................... 1339, 1979

Li, Ker-Chau ................................... 878

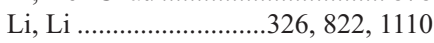

Li, Long.......................................... 722

$\mathrm{Li}, \mathrm{Ma}$.............................................. 702

Li, Min .............................. 289, 1450

Li, Ming ....................................... 311

$\mathrm{Li}, \mathrm{Na}$........................................... 1542

Li, Nim............................. 1097, 1098

Li, Qing Kay ............................... 1875

Li, Shaoying .......... 1396, 1416, 1419 ,

$1456,1492,1525$

Li, Ting....................................... 1403

Li, Wan-Shan................................... 76

Li, Weihong...................................... 417

Li, Xiaodong ................................ 1170

Li, Xiaoqiu ....................................... 1403

Li, Xiaoxian ........ 195, 200, 238, 242 ,

243

Li, Xiaoxian (Bill)........ 207, 208, 209

Li, Xinran ..................................... 1571

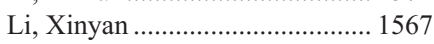

Li, Xuechan.................................. 1121

Li, Yan.............................. 1377, 1416

Li, Yi ............................................ 1417

Li, Yu-I......................................... 723

LI, Yunjie ...................................... 980

Li, Zaibo...... 172, 188, 189, 190, 210 , $401,482,484,724$

Li, Zhongwu......................... 713, 876

Liang, Cher-Wei............................. 1168

Liang, Li...........................1171, 1172

Liang, Peir-In ............................... 1291

Liang, Sharon................... 1143, 1218

Liang, Sheng-Ben ................ 829, 834

Liang, Shu ....................................... 1143

Liang, Xiayuan.............................. 1485

Liang, Yaoming............................... 472

Liao, I-Chuang .............................. 76

Liao, Jie............ 577, 666, 1532, 1635 ,

1636,1652

Liao, Wei-An

1343
Liao, Wei-Li .................................. 1813

Liao, Xiaoyan........... 478, 1980, 2022

Lichner, Zsuzsanna ........................ 981

Lidke, Diane...................................... 1371

Lieberman, David B ...................... 1796

Liegsalz, Veronika........................... 206

Lifton, Richard P......................... 1453

Ligon, Azra H.......... 1144, 1379, 1696

Ligon, Keith L.................. 1696, 1720

Liles, Darla...................................... 1491

Lim, Diana ..................................... 1173

Lim, Jeffrey Chun Tatt ................... 176

Lim, Jeffry Chun Tatt...................... 104

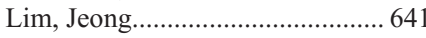

Lim, Megan S................... 1313, 1439

Lim, Raymond ............................... 268

Lim, Soon-Thye ........................... 1489

Lin, Caroline M..................................... 418

Lin, Chieh-Yu................................. 211

Lin, Chung-Wu ............................ 1527

Lin, Daniel ..........................993, 1060

Lin, Doug I.....................................1118

Lin, Douglas.................................... 1174

Lin, Fan ........... 408, 650, 1695, 1960 , 2054

Lin, Fritz ……………………....... 121

Lin, Grace Y ................................... 1980

Lin, Henry .......................... 1539, 1571

Lin, Jingmei ...... 652, 717, 759, 1637 ,

1680

nathan H........................... 1731

Lin, Ming-Chieh ........................... 1168

Lin, Ming-Tseh ................. 984, 1854

Lin, Oscar......... 363, 418, 1318, 1580 ,

$1581,1827,1954,2075$

Lin, Pei........ 1341, 1385, 1395, 1424 ,

1492,1525

Lin, Peter T ............................... 4, 314

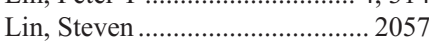

Lin, Xiaoqi .......... 382, 419, 577, 1635

Lin, Yi ……........................................... 691

Lin, Yung-Chang .................... 173, 297

Linares, Emma ........... 527, 677, 1418

Lincoln, Mark …………………........ 553

Lindberg, Matthew R ......................685

Lindeman, Neal........... 431, 599, 801,

$1144,1733,1757$

Lindholm, Kaleigh E...................... 443

Lindsey, Kathryn............................ 448

Lindstrom, Linda S .............. 121, 122

Lingen, Mark...................................... 1309

Link, Brian ..................................... 1363

Liomba, N George......................... 1362

Lipkin, Steven .............................. 1828

Lira, Maruja E................................... 312

Lis, Rosina .................................. 1078

Lisovsky, Mikhail ........................... 756

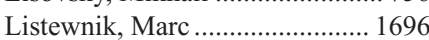

Litzow, Mark R ………………... 1514

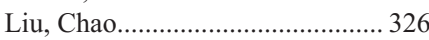

Liu, Chen ......................................... 647

Liu, Cheng Z ......................... 604, 616

Liu, Chengbao............................... 1947

Liu, Chia-Jen ..................... 1278, 1805

Liu, Haiyan ................. 408, 1695, 2054

Liu, Hui .................... 212, 1368, 1457

Liu, Huifei................................... 1465

Liu, Jen-Jane ...................................... 856

Liu, Jingxuan.................................. 1947

Liu, Jinsong........................1171, 1172

Liu, Li ........................................... 725
Liu, Liping .................................. 1884

Liu, Qingqing ........................ 726, 982

Liu, Rangjiao.................................. 1568

Liu, Shiguang ..................................... 318

Liu, Shu-hong ………………..... 1542

Liu, Shuhong................................... 338

Liu, Su-Yang ............... 213, 214, 727

Liu, Ta-Chiang ............................... 709

Liu, Ting Ting ………………….... 1671

Liu, Tzu-Ying ................................. 988

Liu, Wei.............................................. 1419

Liu, Xiaoying ... 358, 756, 1770, 1787

Liu, Xichun

Liu, Xiuli........... 632, 796, 835, 1227 ,

$1662,1663,1664$

Liu, Xulei ............................331, 337

Liu, Yajuan ........................................ 88

Liu, Yen-Chun.......... 1373, 1420, 1473

Liu, Yifang ..................................... 136

Liu, Yuan......................................... 260

Liu, Yuhua .......................................... 88

Liu, Yuxin.......................................1113

Liu, Zach ............................ 983, 1081

Liverman, Christopher ................ 1175

LiVolsi, Virginia ................... 605, 1296

LiVolsi, Virginia A .......................... 589

Ljung, Britt-Marie....... 365, 388, 431,

Llanos, Cesar A $\quad \begin{array}{r}595 \\ \quad 1550,2016\end{array}$

Lloret, Adria ................................. 2024

Lloreta, Josep ...... 893, 947, 948, 960, 2024, 2045, 2055, 2077

Lloveras, Belen ………………..... 1194

Lloyd, Isaac......................... 728, 1607

Lloyd, Ricardo V......... 569, 607, 615,

Lo, Amanda.................................. 1421

Lo, Amy A......................... 1758, 1807

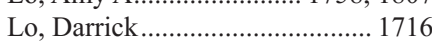

Lo, Ying-Chun ................................ 511

Lo, Yun-Feng ........................ 173, 297

Lo, Yungtai............................ 174, 222

Lobo, Joao........................................ 258

Lockhart, Valerie........................... 1981

Lockyer, Megan G ........................ 420

Loda, Massimo............................... 1065, 1078

Loeb, Keith ..................................... 879

Loftus, Barbara ............................. 1977

Logan, Patrick .................. 1736, 1737

Loghavi, Sanam ............... 1422, 1464

Logothetis, Christopher J ............ 1061

Lohse, Christine M......................... 803

Lokhandwala, Parvez M ............... 984

Lomazzi, Sandra .......................... 1698

Lombard, David ............................... 91

Lombardo, Kara A....... 678, 823, 827, 973, 1069, 1324, 1378, 1729, 1793

Lomme, Michele M ................... 1196

Long, Bradley .................................. 710

Long, Steven R............................... 1973

Long, Susan

1829

Long, Tiffany I

1092

Longacre, Teri A................... 413, 423, $449,729,730,1093,1183,1219$,

1237,1781

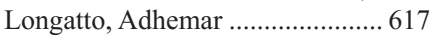

Longerich, Thomas ........................... 1657

Lopategui, Jean ................. 1815, 1872

Lopes, Ana .................................. 1952

Lopes, Jose M .............................. 1203

Lopes, M Beatriz.................... 27, 587
Lopez-Beltran, Antonio................ 1011

Lopez-Guerrero, Jose Antonio ... 1197

Lopez-Janeiro, Alvaro .................. 1706

Lopez-Picazo, Jose M .................... 421

Lopez-Terrada, Dolores ............... 1399

Lopez, Ariana .................................. 228

Lopez, Giselle ………................. 1715

Lopez, Jose I .................... 1010, 1062

Lopez, Mae …………………...... 1984

Lopez, Mirtha....................... 199, 2049

Loren, Alison W ............................ 1514

Lorente, Jose A............................. 2055

Lorenzo, Marta.... 893, 947, 948, 960,

$\begin{array}{rr} & 2055 \\ \text { Lorenzoni, Cesaltina } & 1535\end{array}$

Losada, Daniele M...................... 866, 867

Losada, Hector ................... 1763, 1764

Lotan, Tamara ..... 849, 930, 931, 970,

1060

Lotan, Yair..................................... 1032

Louie, Christine Y ............... 729, 730

Loundas, Craig ................................ 548

Lovane, Lucilia ................................. 1535

Love, Cassandra ........................... 1354

Lovisolo, Silvana M........................... 617

Lovitch, Scott B ....... 402, 1332, 1379

Low, Lawrence.................. 1329, 1423

Lowe, Alarice ................................. 458

Lowe, Chenery ............................. 2067

Lowe, Lori........................................ 487

Lowe, Scott ……………………...... 50

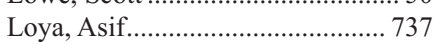

Lozano, Maria D ............................ 421

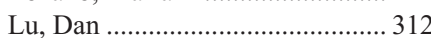

Lu, David Y...........................226, 991

Lu, Fang-I ..... 167, 1087, 1097, 1098 ,

2071

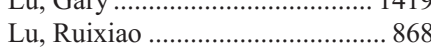

Lu, Shaohua .................... 1885, 1886

Lu, Shaolei ........... 678, 696, 758, 809, $823,1069,1547,1783$

Lu, Tzu-Pin ................................. 1168

Lu, Xinyan ............. 1385, 1395, 1396 ,

1419,1424

Lu, Zhichun.................................... 985

Lubin, Daniel ....................... 731, 1473

Luchini, Claudio ………………..... 968

Lucia, MS....................................... 954

Ludwig, Kathrin ................................ 1461

Luebker, Stephen ............................... 1799

Luger, Selina M.............................. 1514

Lugli, Alessandro ....................... 662, 663, 664

Lui, Weng-Onn............................... 514

Luinetti, Ombretta................................... 609

Luk, Adriana .................................. 320

Luk, Peter P.................... 1292, 1293

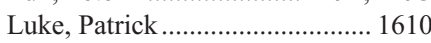

Lum, Amy ………………………....... 44

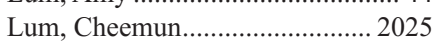

Lum, Trina............... 1292, 1293, 1308

Luna, Eric............................ 352, 1970

Lunz, John..................................... 1616

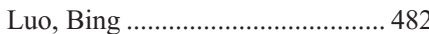

Luo, Jun........................................... 931, 984

Luo, Shujun......................1792, 2052

Luo, Yuling........................ 1542, 1875, 2026,

2051, 2085

Luskin, Marlise ............................... 1514, 1515

Luthra, Raja...................... 1705, 1957 
Luthra, Rajyalakshmi......... 444, 534, 721, 1374, 1388, 1389, 1390, 1391, 1464, 2003, 2058

Luthringer, Daniel..... 315, 878, 1030,

Luthringer, Daniel J ...................... 986

Lutterbaugh, James ....................... 822

Luu, Hung S ................................. 1355

Luvison, Alyssa M ..... 354, 605, 1272

Luz, Rita...................................... 1126

Luzar, Bostjan ............. 497, 498, 512

Lyapichev, Kirill ........................... 969

Lydon, Christine........................... 1909

Lyons, Genevieve R.... 150, 307, 371, $488,535,1124$

Lytwyn, Alice................................. 361

Ma, Changqing..................... 709, 775

Ma, Deqin ……………………........... 60

Ma, Fen ……………………....... 1078

Ma, Juan ......................................... 1449

Ma, Lisa ................. 1364, 1392, 1430

Ma, Shuhua ...................................... 549

Ma, Sophia ..................................... 1982

Ma, Xiao-Jun......... 1542, 1875, 2026 , 2051,2085

Ma, Yihong.................................. 1808

Ma, Zheng ....................................... 595

MacDonald, Theresa Y .............. 1002

Macedo, Fabiane C .................... 2038

Macedo, Gabriel S ..... 250, 268, 1250

Macerola, Elisabetta........................ 571

MacEwen, Clare................................. 1622

MacGrogan, Gaetan ...................... 254

Macha, Muzafar A........................ 1322

Machi, Junji............................... 2068

Machinami, Rikuo............................. 93

Maciak, Charlene .............................. 99

Macias, Virgilia ...................... 215, 793

Mackinnon, Alexander C .... 78, 1103 , 1107,1617

Mackrides, Nicholas ........ 1202, 1425

MacLennan, Gregory ... 843, 873, 958

MacLennan, Kenneth.................. 1454

Macon, William R............ 1408, 1426

Madabhushi, Anant ........... 269, 1561, $1562,1566,1583$

Madan, Rashna............................... 364

Madan, Shashi.............................. 273

Maddala, Tara.................................. 989

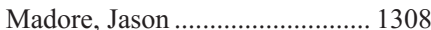

Madory, Jim ...................................... 550

Madrigal, Emilio ……………….......554

Maeda, Daichi ..................... 844, 1176

Maeda, Nagako …………………... 405

Maedler, Chelsea............................. 732

Maestro, Roberta................................ 84

Mafnas, Chrisy T.......................... 1973

Mafolasire, Abiodun ........................ 841

Magaki, Shino ............................... 1716

Magers, Martin J ........... 422, 987, 988

Maggioni, Marco.......................... 1865

Magi-Galluzzi, Cristina........ 864, 865, 905, 957, 989

Magid, Margret S ........................... 837

Maglantay, Remegio ........................ 1569

Magliocco, Anthony M ....... 749, 895,

1221,1240

Magno, Crystal............................ 1670

Magnoli, Francesca ..................... 1500

Magro, Cynthia ............ 517, 518, 538

Maguire, Donal ............................... 658
Mahadevan, Krishnan K ..... 667, 782,

1747

Mahadevan, Navin ....................... 1887

Mahaffey, Alyssa................... 464, 1314

Mahajan, Aparna ......................... 1257

Mahalingam, Meera .............. 502, 516

Mahan, Sarah .............................. 1251

Mahantshetty, Umesh M ................ 995

Mahe, Etienne ………………….... 1361

Maheshwari, Priyanka.................... 533

Mahlow, Jonathon .............................. 588

Mahmoud, Amr ………………........ 733

Mahon, Sarah ................................ 1177

Mai, Kien T ......... 400, 860, 861, 862,

950

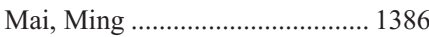

Maier, Cheryl ………………….......559

Maietta, Antonio ………………...... 343

Main, Nelson................................... 1961

Mainar, Margarita ………………. 1577

Maisel, Julia R ...............................589

Maisonneuve, Patrick.................... 591

Maithel, Shishir....... 772, 1763, 1764,

1766,1767

Maitra, Anirban ................... 726, 750

Majeed, Hassaan ............................ 215

Majewski, Jacek ................................. 54

Majewski, Tadeusz............... 891, 935

Makowsky, Robert ..................... 1092

Malafa, Mokenge P.... 749, 816, 1744

Malapelle, Umberto .............. 465, 808

Malcolm, Joan............................... 2046

Maldonado-Duran, Mayra............. 245

Maldonado, Leonel ......................... 555

Maleki, Zahra... 366, 812, 1883, 1916

Malenie, Renuka ........................... 549

Maleszewski, Joseph J ...... 4, 15, 314, $319,324,325,1855$

Maletta, Francesca ................ 569, 618

Malhotra, Saurabh...........................695

Maliakkal, Benedict ..................... 1642

Mallon, Abbie ............................... 2010

Maloney, Kuda .................................565

Malpica, Anais ..........523, 1124, 1125, $1178,1199,1209,1253$

Maluf, Horacio M ........................ 1091

Mamut, Adiel ................................ 1610

Manca, Rachele................................. 609

Mancini, Irene ....................................56

Mandal, Rakesh.................................. 607

Mandavilli, Srinivas......................... 356

Manekia, Jawad....... 444, 2003, 2058

Mangray, Shamlal ....... 758, 809, 827, $973,974,1729,1793$

Mangus, Richard S........................ 652

Maniar, Kruti.................383, 430, 1169

Manivel, Juan Carlos ....................... 20

Manna, Pradip ..................... 975, 1088

Mannan, Abul Ala Syed Rifat ... 1294

Manning, Suzanne........................... 155

Manning, Tim.................... 118, 1574

Mano, Masayuki .............................. 72

Manoj, Namitha .......................... 1170

Mansfield, Aaron S.......................... 529

Mansfield, James............................. 2056,779

Mansfield, Paul .....................698, 779

Mansoor, Adnan ................... 1361, 1417

Mansoor, Atiya............................. 1830

Mansoor, Mohammad O ............. 1469

Mansoor, Was................................ 1897

Mansour, Mena ................... 734, 1295
Mansukhani, Mahesh ........ 694, 1401, 2079

Mantilla Arango, Jose ................... 216

Mantilla-Morales, Alejandra ......... 850

Manzano, Jose Luis....................... 746

Mao, Mao ...................................... 312

Mao, Yong.................................... 308

Maque-Acosta, Yvan................... 1425

Marafioti, Theresa ....................... 1483

Maragliano, Roberta ........... 585, 609

Marcano, Eugenio .......................... 245

Marchand, Vinciane ....................... 38

Marchetti, Elizabeth...................... 225

Marchevsky, Alberto M ........... 1869, $1872,1934,1935$

Marchio, Caterina ....... 160, 217, 268,

Marco, Marta.............................. 1084

Marcus, Alan .................... 1255, 1944

Mares, Roso ..................................... 49

Margolskee, Elizabeth...... 1427, 1455

Marguet, Florent.......................... 1809

Margulis, Vitaly ............................. 913

Mariani, Andrea ........................ 1225

Maric, Irina ........... 1356, 1507, 1519

Marimon, Lorena ....................... 1220

Marino-Enriquez, Adrian ................ 77

Mark, Eugene J ......................... 1888

Mark, Tomer............................... 1360

Marketkar, Shivali............. 218, 1983

Markowitz, Sanford ..................... 822

Marks, Randolph S......................... 1911

Marotti, Jonathan ......... 148, 232, 358

Marques, Sara ............................ 1952

Marrades, Ramon....................... 1907

Marrero, Bernadette .................... 1520

Marsh, William L ........................... 724

Marshall, Carrie B........................ 438

Marshall, Hyejong S ...................... 17

Martelotto, Luciano G........ 250, 268,

1250

Marti, Carles ............................. 1289

Marti, Gerald............................. 1507

Martignoni, Guido..... 873, 968, 1074

Martin-Algarra, Salvador............. 421

Martin, Brock A ............................ 423

Martin, Cara ........... 437, 1099, 1243,

1251,2034

Martin, Cara M............... 1317, 1820

Martin, Cheyenne........................ 1371

Martin, Cristina ................................ 543

Martin, David.......... 777, 1759, 1811

Martin, Jennifer........................... 2012

Martin, Samantha A ................... 1933

Martin, Sean T.............................. 680

Martinek, Petr .................. 1010, 1027

Martinez de Ubago, Julianne .... 1204,

1205

Martinez Duarte, Ernesto ............. 610

Martinez-Cardus, Anna ................... 746

Martinez-Duarte, Ernesto............ 1206

Martinez, Anthony .................. 36, 48

Martinez, Kimberly...................... 380

Martinez, Laura.............................. 543

Martinez, Miguel J........................ 1535

Martinez, Paola .......................... 1813

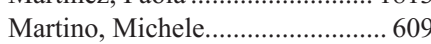

Maru, Dipen ...................... 622, 623

Marukawa, Katsuji ..................... 1760

Maruyama, Riruke ...................... 735

Masand, Ramya P .................... 1167
Maset, Maria Amada.................... 421

Masia, Ricard ............................ 1630

Mason, Emily .............................. 736

Masood, Muhammad A................. 737

Massard, Christophe .................. 1822

Master, Stephen R ............ 1514, 1515

Master, Viraj A ............................. 898

Masterson, Timothy A................... 941

Masugi, Yohei ........................... 1673

Mata, Douglas A........................... 990

Mateescu, Valerica ............ 424, 1853

Mateo, Joaquin................ 1001, 1002

Mates, Mihaela............................ 1939

Mather, Cheryl .......................... 1671

Mather, Cheryl A........................ 1761

Mathew, Susan ...... 1373, 1468, 1473,

1473,
1817

Mathur, Priya ................................. 793

Matias-Guiu, Xavier .................. 1199

Matias, Gilberto .......................... 1193

Matias, Pamela R ...................... 1917

Matos, Ana ................................... 1193

Matos, Rute .................................. 1952

Matoso, Andres ... 809, 904, 928, 973, 974, 975, 977, 979, 991, 1793

Matrai, Cathleen.............. 1179, 1838

Matsubara, Osamu ..................... 1888

Matsuda, Midori............................ 598

Matsuda, Yoko ............................... 333

Matsukuma, Karen............... 687, 755

Matsumoto, Melissa..................... 456

Matsumoto, Seiichi ........................ 93

Matsumoto, Shinji..................... 1868

Matsumoto, Toshiharu ................. 738

Matsunaga, Rie ............................ 739

Matsuno, Yoshihiro ..................... 1760

Matthew, Stemm ............................... 78

Mattia, Antony .............................. 667

Maurer, Matthew........................... 1363

Mauzo, Shakuntala................... 1428

Maxfield, Erin .............................. 1905

Maxwell, Jessica E...................... 568

Mayigowda, Pramod....... 1539, 1571

Mazzaferro, Vincenzo .......... 591, 592

Mazzoli, Sandra ........................ 1003

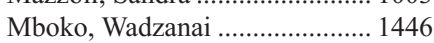

Mc Dermott, Ray ........................ 680

McAlpine, Jessica N .......1131, 1132, $1139,1140,1152,1236$

McCall, Brant................................ 447

McCall, Chad M............... 1376, 1502

McCall, Shannon J....................... 688

McCann, Amanda .......................... 1099

McCann, Brooke...................... 1618

McCarthy, Aoife........ 126, 1717, 1718

McCarthy, Julie................ 2011, 2012

McCarty, Tom ............................ 1659

McCash, Samuel I.......................... 1954

McCleskey, Brandi C................... 991

McCluggage, Glenn ....................... 1229

McCluggage, W Glenn ....1186, 1199,

1207

McConechy, Melissa........1131, 1132,

1140

McConkey, David ................ 891, 935

McCormack, Gina..................... 1959

McCormick-Baw, Clare ................ 1984

McCoy, Lucie............................. 1725

McCroskey, Zulfia................ 425, 426

McCuiston, Austin M................. 1429

McCullough, Arthur................... 1662 
McCullough, Debbie............. 113, 868

McDaniel, Andrew ........................ 988

McDonald, Anna G ..................... 1195

McDonald, Robert........ 144, 219, 286

McDonald, Seamus ...................... 1191

McDonald, William.......................... 1724

McEnery-Stonelake, Melissa ....... 509

McFadden, Julie......................... 1177

McGinnis, Lisa........ 1430, 1431, 1973

McGovern, Brianan...................... 1762

McGrath, Patrick C ...... 144, 219, 286

McGraw, Kathy ........................... 1444

McGregor, Stephanie .......... 220, 221,

1935

McHugh, Jeffrey ....

1557

McHugh, Jonathan ........... 1048, 1268,

1278,1313

McHugh, Kelsey ............... 1148, 1889

McIntosh, Rebekah ...................... 992

McKee, Thomas ............................ 1302

McKee, Trevor .............................. 1774

McKenna, W Gillies ................... 1779

McKenney, Jesse K.............. 506, 905, 957, 993, 1011, 1060, 1062, 1066

1074

McKeon, Frank D ....................... 1252

McKinnon, Elizabeth L................... 513

McKissic, Devin ........................... 581

McLaughlin, Sarah A ... 229, 230, 305

McLemore, Lauren E .................... 222

McMahon, Kelly Q ......................... 624

McMahon, Loralee...... 640, 857, 858 ,

McManus, Bruce M ........................ 321

McNally, Scott ............................. 339

McNeal, Sa'Rah McNeal ............ 1095

McNeer, Jennifer........................... 1504

McNeish, Iain............................... 1100

McNett, Henry ................................. 514

McNiff, Jennifer M .............. 247, 505

McWilliams, Robert R .... 1684, 1685,

1756

Medeiros, Jeffrey ............ 1347, 1377, $1416,1419,1464,1465,1521$

Medeiros, L J................... 1522, 1525

Medeiros, L Jeffrey ............ 444, 1341,

$1368,1385,1395,1396,1422,1424$, 1456, 1457, 1492, 1509, 1526,

2058

Medieros, Bruno C........................ 1350

Medlin, Erin E............................... 1257

Medline, Paul .............................. 1540

Meeker, Alan...... 293, 856, 883, 1754

Mehr, Chelsea ………………….... 557

Mehra, Niven .................... 1001, 1002

Mehra, Rohit ... 847, 988, 1048, 1065,

1066

Mehrad, Mitra ....... 1890, 1891, 1892 ,

1930

Mehran, Reza J....... 1873, 1874, 1931

Mehrotra, Meenakshi ................. 2058

Mehrotra, Swati...... 1923, 1945, 1991

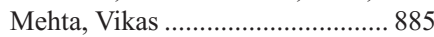

Meijer, Sybren L ............................. 811

Meinke, Kyle.................................... 793

Meireles, Catarina ......................... 1203

Mejia, Jaime ...................................... 816

Mejias Sosa, Luis D ..................... 1706

Melamed, Jonathan ..... 881, 949, 966, $977,978,979$

Meliti, Abdelrazak.... 994, 1029, 1054
Melnick, Ari M.

1514

Meltzer, Paul ................................. 1803

Memis, Bahar...... 392, 393, 636, 773,

$826,1738,1740,1748,1759,1763$, $1764,1765,1766,1767,1772$,

1784

Mendoza Rodriguez, Maria Angelica ..... 886, 887, 934, 1004

1053

Mendrinos, Savvas..... 977, 979, 1041

Menegaux, Fabrice........................... 593

Menei, Philippe ……………….... 1727

Menéndez, Clara ............................ 1535

Meng, Bin ................................... 1352

Meng, Xiuling ............ 509, 885, 1523

Mengel, Michael ............... 1585, 1627

Menon, Madhu .............................. 1369

Menon, Mani................................. $955,956,985$

Menon, Santosh............................ 995

Merchant, Nipun B.......................... 469

Merino, Maria J................. 1717, 1718

Merli, Michele.............................. 1500

Merrill, Parker................................ 1696

Merzianu, Mihai....... 193, 1264, 1985

Mesa, Hector .................................... 20

Meschter, Steven ............................. 409

Meserve, Emily .........223, 1118, 1180

Messenger, David E ....................... 675

Messing, Edward M ...................... 965

Mete, Ozgur .......................................... 3

Meunier, Danielle.............................590

Meyer, Rose .................................. 2000

Meyers, Carolyn A ........................... 79

Meyerson, Howard J ......... 1326, 1499

Mezhir, James J............... 1745, 1778

Mian, Badar.................................... 842

Micevic, Goran ............................. 2074

Michael, Claire W ........ 379, 391, 406

Michael, Helen E ........................... 942

Michal, Michael ............................... 81

Michal, Michal .......... 81, 1010, 1027,

Michalak, Sophie _........... 11727

Michalowski, Susan ........................ 1369

Middha, Sumit.............. 34, 795, 2075

Middleton, Lavinia.......................... 224

Mierau, Gary ................................. 2043

Mies, Carolyn.................................. 113

Miettinen, Markku ........................ 1862

Migliacci, Jocelyn C ................... 1320

Migliore, Federico............................. 330

Mihalov, Mike................................. 702

Mihm, Martin C .............................. 528

Mikael, Leonie G .............................. 54

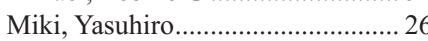

Miles, LiLi ..................................... 1719

Miles, Michael .............................. 1719

Miley, Timothy G......................... 1432

Milikowski, Clara ................... 17, 778

Milione, Massimo ………….... 591, 592

Millan, David ................................. 1100

Millan, Rosana ............................... 1577

Miller, Benjamin ............................... 60

Miller, Caitlyn ................................ 1272

Miller, Dianne M............................. 1152

Miller, Dylan .... 205, 339, 1607, 2044

Miller, Gregory ................... 740, 741

Miller, James A …………………...... 366

Miller, Melanie.................. 2026, 2051

Miller, Michael................................ 757

Miller, Michael B ........................ 1720
Miller, Naomi A

1824

Miller, Ross A ………………….... 427

Miller, Sue..................................... 1438

Miller, Todd...................................... 232

Miller, Vincent A......... 146, 241, 271, $528,611,842,1025,1123,1260$, $1287,1505,1699,1785,1795$,

1927

Mills, Anne M ..........162, 1181, 1182, 1231,1700

Mills, Gordon............ 199, 846, 1044 , 1164,2049

Mills, Stacey E .................................... 605

Milman, Tatyana .......................... 1296

Milne, Katy .......................... 794, 1919

Milner, Danny A............................. 223

Milton, Denai R ........... 490, 530, 540

Min, Kyueng-Whan ........................ 194

Minami, Yuko............................... 1920

Minca, Eugen C ............................ 515

Minden, Mark …………………... 1393

Miniti, Caterina ............................ 1520

Minkovsky, Alissa ......................... 1433

Mino-Kenudson, Mari..... 1757, 1871,

1920

Mino, Barbara ....... 1931, 1937, 2080 2081

Mir, Saad ....................................... 1720

Miranda, Alonso........................... 1457

Miranda, Roberto ... 1368, 1465, 1526

Miranda, Roberto N .................... 1457

Miranda, Susana AA ......... 1001, 1002

Mirkovic, Jelena................. 428, 1180

Miron, Alexander ................ 822, 1234

Mirsadraei, Leili............................ 478

Mirza, Kamran ............................ 1434

Mirza, Kamran M................ 542, 1945

Mirza, M Kamran.......................... 1328

Misumi, Kento ................... 1660, 1661

Mitchell, Brendon ........................... 516

Mitchell, Kisha.................... 654, 1659

Mitchell, Mary J............................ 1579

Mitchell, Richard N ..................... 320

Mito, Jeffrey................................. 742

Mitsios, John V .......................... 1468

Mitsuhashi, Tomoko........................... 1760

Mittal, Karuna ................................ 2059

Mittal, Khushbakhat.................... 1255

Mittal, Pardeep...... 1748, 1765, 1766,

1767

Mittendorf, Elizabeth A....... 106, 150

Miura, Tomisato ........................... 1573

Miyamoto, Hiroshi ............... 961, 963

Miyaoka, Youichi ............................ 735

Miyauchi, Akira ................................597

Mizrahi, Meir ................................ 692

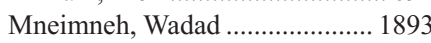

Mo, Angela Y .................................. 321

Moatamed, Neda A............. 480, 1211 ,

1212

Moch, Holger ....................... 298, 1074

Mochizuki, Kunio ........................... 597

Mockus, Susan ................. 1568, 1810

Modesitt, Susan C .......................... 1181

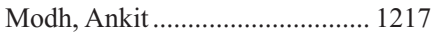

Modiano, Nir.................................... 641

Mody, Dina R............ 352, 427, 1970

Mody, Roxanne R ............... 352, 1970

Moeckel, Gilbert ............... 1598, 1628

Moeller, Inga ..

.... 1721
Moench, Laura ................. 1183, 1237

Moffitt, Andrea............................ 1354

Moh, Michelle............................... 1435

Mohamed, Nehad......................... 1829

Mohammad, Amin ............. 566, 1984

Mohan, Helen........................................ 680

Mohanty, Suravi ............................ 1831

Moises, Jorge …............................ 1907

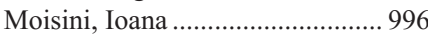

Moiyadi, Aliasgar.......................... 1701

Mojica, Gregg ............................. 1986

Mojica, Paul ...................... 225, 2060

Mojica, Wilfrido....... 225, 1986, 2060

Moldovan, Cosmin......................... 287

Moldovan, Krisztina .......................... 1728

Molinaro, Annette ........................ 1725

Moline, Teresa............................ 49, 83

Molins, Laureano .......................... 1907

Mollejo, Manuela .............................527

Moller, Henrik............................... 863

Moller, Michael B …………….... 1522

Molnar, Stacy L............................. 417

Molofsky, Ari ............................... 1513

Molony, Peter .................................... 18

Momblan, Dulce ................................. 2042

Momeni Boroujeni, Amir...... 19, 704,

Momtahen, Shabnam ...........517, 518

Monaco, Sara E.......... 355, 464, 1575

Monaghan, Sara A.............. 558, 1349

Moncea, Denisa............................... 287

Mondal, Ashis K ........................ 508, 1409, $1711,1804,2031$

Monges, Genevieve....................... 671

Montahen, Shabnam .................... 538

Montalto, Michael....................... 1582

Montaser, Laleh ............................... 1078

Montealegre Sanchez, Gina ....... 1520

Montecalvo, Joseph...................... 1894

Montemayor-Garcia, Celina.......... 607

Montes-Moreno, Santiago........... 1418

Montes, Santiago................. 527, 1197

Montgomery-Goecker,

Crystal ................................. 1346

Montgomery, Elizabeth A ... 681, 703, $768,769,771$

Montgomery, Jeffrey S................. 988 Montgomery, Nathan .................. 1362

Montilla, Silvia ........................... 1917

Montironi, Carla................. 630, 2042

Montironi, Rodolfo ............. 873, 1008

Montone, Kathleen...... 548, 557, 589,

1298

Monzo, Mariano.............................................. 1956

Moon, Andres.................................. 1609

Moon, Kyung Chul ..................... 1015

Moon, Kyung-Sub............................. 1882

Mooney, Kelly................................ 226

Moore, Erika M................................ 1436

Moorhead, Jane ................... 897, 1794

Morabito, Anna ............................. 1861

Morais, Carlos L ........ 849, 930, 931,

1060

Moran, Cesar A ..... 1873, 1874, 1906, $1931,1936,1937$

Moran, Elisa J ............................. 1526

Moran, Miriana ........................... 1092

Moran, Sebastian.............................. 746

Morandi, Luca.................................. 569

Morash, Chris....................... 862, 950 
Moreira, A .................................. 1894

Moreira, Andre....... 1910, 1920, 2075

Moreira, Roger....... 1643, 1665, 1666

Morellas, Vassilios ........................ 1567

Morency, Elizabeth ............ 430, 1853

Moreno-Aspitia, Alvaro ...... 229, 230 ,

305

Moreno, Carlos .............................. 1811

Moreno, Debora ............................. 1813

Moreno, Vanessa ............... 1717, 1718

Morey, Gabriel E............................. 997

Morgan, Elizabeth A .................... 1433

Morgan, Margaret B....................... 846

Morgan, Terry K............ 372, 420, 641

Morgan, Todd................................. 988

Morgen, Eric ................................ 1570

Mori, Yukiko …………………..... 1063

Morice, William G ............ 1338, 1442

Morikawa, Landon ............................ 463

Morikawa, Teppei ........................... 619

Morita, Kouhei ................................ 236

Morita, Michi ................................ 227

Moritani, Suzuko..............................2236

Morley, Samantha ……………… 1505

Morlote, Diana ................................ 228

Morooka, Asami........................... 1760

Morote, Juan ................................... 894

Morotti, Raffaella........................ 1843

Morra, Massimo................ 1792, 2052

Morris Faltas, Bishoy S............... 1056

Morris, Catherine S ........................ 743

Morris, Elizabeth.................... 252, 277

Morris, Robert T ……………..... 1084

Morrissette, Jennifer JD ............... 1473 ,

1639,1796

Moscinski, Lynn C........... 1400, 1444,

1528

Moser, Justin ..................................529

Moskaluk, Christopher A ............ 1181,

1231

Moskowitz, Alison J.................... 1375

Mosquera, Juan Miguel......... 23, 440, 889, 1002, 1024, 1056, 1816, 2035

Mosse, Claudio A................................ 7

Mostafa, Mohamed ......................... 744

Mosunjac, Marina .............. 1964, 1965

Mosunjac, Mario ……………........ 1965

Motoi, Noriko .......................93, 1915

Mott, Sarah L .............................. 1745

Moukarbel, Roger ............ 1259, 1276

Moul, Adrienne ...... 1425, 1437, 1490

Mounajjed, Taofic ... 714, 1645, 1665 $1684,1685,2093$

Moura, Caio M.............................. 998

Mourra, Najat................................... 593

Moussa, Madeleine .............916, 1610

Moy, Andrea............................................ 519

Moyer, Ann M.............. 229, 230, 305

Mozos, Anna ............................... 1483

Mpunga, Tharcisse ............................ 223

Mrak, Lara N.................................... 90

Mroz, Pawel ..................... 1438, 1439

Mucci, Lorelei A ......................... 1065

Mucientes, Francisco ....... 1738, 1772

Mudaliar, Kumaran ............. 520, 1987

Mudalige, Tudor.......................... 1872

Mueller, Jeffrey .............................. 231

Muezzinoglu, Bahar..................... 1184

Muhlbauer, Aaron ................. 517, 518

Muir, Jennifer................................. 1540

Mukherjee, Somnath ..................... 1779
Mukhopadhyay, Sanjay.... 1889, 1895

Mullaney, Kerry ................................ 57 Mullen, Dorinda ................. 1099, 1243

Muller-Hermelink, H Konrad .... 1454

Muller, Kristen ............................... 232

Muller, Sarina................................. 1258

Mulligan, Anna Marie.................. 1967

Mullighan, Charles G................... 1791

Munday, William............................... 521

Mungall, Andy ................................. 846

Munkarah, Adnan R .......... 1083, 1089

Munne, Jessica .............................. 2024

Munoz, Nubia .......................................... 871

Muppa, Prasuna.................... 745, 999

Murakami, Keigo ............................ 583

Muraki, Takashi ........ 772, 826, 1748, 1763, 1764, 1765, 1766, 1767

Murali, Mandakolathur ................. 519

Murali, Rajmohan ...1185, 1186, 1721

Muralidhar, Akshay ...................... 1923

Murase, Yota .................................... 405

Murgic, Jure ........................................ 1067

Murillo, Lena ................................. 1394

Murphy, Claire E.............................. 759

Murphy, Cliona .................. 1191, 1243

Murphy, Jean...................... 658, 1762

Murphy, Joanne............................... 732

Murphy, Noel ..................... 118, 1574

Murphy, Rose............................... 127

Murphy, Sandra...............................524

Murphy, Tom................................... 733

Murray, Arlene .................................. 361

Murray, David ............................... 1987

Murray, Melissa .......... 252, 259, 268,

277,1823

Murro, Diana................................... 594

Murthy, Jayasimha N ........ 696, 1146,

Murthy, Kruthi ............................ 1383

Murthy, Stacey L.......................... 1812

Murthy, Vedang .............................. 995

Murugan, Paari......... 606, 1000, 1988

Murugesan, Karthikeyan............. 1571

Muscarella, Peter.......................... 2072

Muschel, Ruth ............................... 1779

Music, Jodi S................................... 233

Musulen, Eva ................................. 746

Muthukumarana, Palawinnage

Vidarshi U .................................. 234

Mutter, George L...........................1118

Mutter, Robert W ............................... 154

Muvugabigwi, Gaspard.................. 223

Muzikansky, Alona ...................... 1920

Muzzafar, Tariq..... 1374, 1388, 1389,

1390,1391

Myers, Charles ......... 435, 1989, 2029

Mylander, W Charles ..................... 205

Myles, Jonathan .............................. 957

Myles, Nickolas .................. 281, 1572

N Kalimuthu, Sangeetha ................ 747

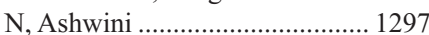

Na, Hee-Young................................ 522

Naab, Tammey ..................... 187, 235

Nabais, Henrique........................... 1193

Nabeshima, Kazuki ...................... 1866, 1868

Naccache, Samia ............................. 869

Nadal, Alfons .................... 1289, 1837

Nadasdy, Gyongyi....................... 1620

Nadasdy, Tibor .................. 1591, 1620

Nadji, Mehrdad .................... 301, 1202

Naeim, Magda........ 1087, 1097, 1098
Naeini, Yalda B ..... 1440, 1517, 1518, 1896

Nafa, Doudja ............................... 2062

Nafa, Khedoudja ............................. 34

Nafisi, Houman .................. 167, 2071

Nagarajan, Priyadharsini..... 488, 490, $523,530,535,540$

Nagel, Barbara ............................. 767

Nagrale, Vidya ............................... 191, 192

Nagy, Peter L............................. 2079

Nagymanyoki, Zoltan .................. 431

Nahar, Niru.................................. 1990

Nahas, Willian C ........................ 1006

Naidoo, Jarushka......................... 1910

Naini, Ali B .............................. 2079

Naini, Bita V ............ 634, 686, 1694

Nair, Ranju .................................. 1824

Nairismagi, Maarja-Liisa ............ 1489

Naizhen, Xu ................... 1717, 1718

Najafian, Behzad .............. 1591, 1624

Najarian, Robert........ 692, 748, 1739

Najfeld, Vesna .............................. 1279

Nakai, Tokiko................................. 236

Nakakura, Eric K ...................... 1755

Nakamura, Shigeo...................... 1359

Nakamura, Takuro........................... 98

Nakamura, Toru ........................... 1760

Nakamura, Yasuhiro....................... 26

Nakashima, Yasuaki...................... 72

Nakazawa, Tadao .......................... 597

Nakazawa, Takahiro................... 1768

Nakhleh, Raouf E....................... 1331, 1686

Nakitandwe, Joy........................ 1791

Nakonechny, Quentin................. 1140

Nalbantoglu, Ilke............... 754, 1640

Nam, Anna ................................... 1441

Nam, Soo Jeong ........................... 1407

Namiki, Thomas............................ 381

Nandula, Subhadra ........................... 1342

Nanji, Shabin....................1115, 1116

Nanus, David M....................... 1024

Naous, Rana .......... 66, 432, 433, 434

Naovarat, Benjamin ......... 1022, 1023

Nardi, Valentina ......................... 1871

Narra, Krishna............................... 339

Nart, Deniz .................................. 1692

Narula, Navneet ......347, 1817, 1901,

1944

Nasir, Aqsa ........................... 20, 182

Nasr, Samih ..................... 1611, 1618

Nasser, Imad.................................. 1739

Nassiri, Mehdi................. 1354, 1559

Nastic, Denis .............................. 1106

Nathanson, Katherine L ............... 589

Nathwani, Bharat N ................... 1454

Natkunam, Yasodha ......... 1425, 1431

Natu, Ashwini ............................ 1300

Naugler, Christopher........... 338, 1570

Nava Rodrigues, Daniel... 1001, 1002

Navaid, Musharraf ..................... 1491

NavaRodrigues, Daniel ................ 976

Navarro, Alfons .......................... 1907

Nawaf, Cayce............................. 841

Nayak, Anupma.... 179, 180, 237, 267

Nayar, Ritu......... 383, 424, 430, 461,

701,1853

Nayor, Jennifer............................. 801

Nazarian, Rosalynn ..................... 519

Nazeer, Tipu ... 842, 1025, 1082, 1470

Nedved, Drew ............................... 2061

Neelkantan, Nikhil

.. 447
Neely, Cameron.......... 238, 435, 2029

Neema, Pranay .............................. 304

Neff, Jadee L ................................ 1442

Neill, Kevin..................................... 749

Neill, Stewart ................................. 559

Nejati, Reza.................................. 750

Nelius, Thomas ........................... 1068

Nelson, Andrew............................. 452

Nelson, Andrew C......................... 182

Nelson, Brad H.................. 794, 1919

Nelson, Emily R............................ 239

Nelson, Gregg ...............................1111

Nelson, Jonathan ............................. 505

Nelson, Karen ............................... 334

Nelson, Peter S.................. 993, 1060

Neo, Meusia ................................ 1173

Neppalli, Vishala........................ 1443

Neri, Bruce .................................... 1032

Nesbitt, Shannon ........................ 1394

Nesi, Gabriella ............................ 1003, 1062

Netto, George J ... 856, 883, 886, 887, 906, 934, 961, 963, 980, 1004,

1053,1054

Neuberg, Donna .......................... 1144

Neupane, Binod................................ 361

Newcomb, Lisa ............................. 993

Newell, Jordan M............. 1127, 1246

Newman, Scott........................... 1578

Newsom, Kent J .......................... 1187

Newsom, Kimberly J ............ 504, 647

Ng, Charlotte KY ....... 154, 160, 217 , 250, 259, 268, 1250, 1798, 1823

Ng, Dianna ...................... 595, 1541

Ng, Siok-Bian .................... 533, 1489

$\mathrm{Ng}$, Tony............................ 44, 2030

Ngeow, Joanne .............................. 1173

Nguyen, Doreen ......... 886, 887, 1004

Nguyen, Giang H ......................... 538

Nguyen, Johnny ............... 1444, 1501

Nguyen, Justin........................... 1686

Nguyen, Khanh ........................... 1776

Nguyen, Nghia D ........................ 1428

Nguyen, Peter................................. 335

Nguyen, Tan .................................. 215

Nguyen, Tu Dung T ..................... 357

Nguyen, Vi .................................... 79

Ni, Shujuan ......................... 751, 805

Ni, Ying ...................................... 1173

Nickeleit, Volker ........................ 1612

Nicolas, Marlo....................... 380, 596

Nicole, Thomas E........................ 1331

Nicosia, Roberto .......................... 316

Nieboer, Daan .................... 971, 972

Niederberge, Nicholas.................. 407

Niederberger, Philipp ................... 919

Nielsen, G Petur................... 45, 2041

Nielsen, Torsten O........... 44, 50, 281

Niesvizky, Ruben ......................... 1360

Nikiforov, Yuri E...... 604, 605, 1017 ,

Nikiforova, Marina ... 604, 605, 1017, $1415,1463,1722,1775,1776$

1891,1892

Nine, Jeffrey. ... 28

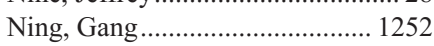

Ning, Jing ..................................... 523

Ning, Yi........................................ 261, 1054

Ninomiya, Hironori..................... 1915

Nishikimi, Toshinori ................... 1063

Nishimura, Michael I ........ 520, 1987

Nishino, Michiya............... 466, 2014 
Nishio, Makoto …………............. 1915

Nishizawa, Yuji .............................. 739

Nitta, Hiroaki ................................ 210

Nitta, Takeo ...................................... 1760

Niu, $\mathrm{Na}$............................1171, 1172

Niu, Shuang.................................. 1188

Nobre, Rui..................................... 1193

Nodit, Laurentia ............................. 385

Nofech-Mozes, Roy ....................... 981

Nofech-Mozes, Sharon ..... 167, 1087, $1097,1098,2071$

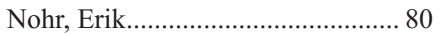

Nolan, Amber .................................... 1189

Nomani, Laila ............ 240, 436, 1445

Nonaka, Daisuke ......................... 1897

Nonaka, Keisuke ............................ 333

Nonell, Lara ....................... 948, 1289

Nordenskjold, Bo ............................ 122

Norkowski, Emma ....................... 1672

Norris, Lucy ......................................... 1191

North, Bernard ................................. 863

North, Paula E................................. 90

Northfelt, Donald W .... 229, 230, 305

Norton, Larry ....................... 253, 268

Nose, Vania ........................... 1306, 2041

Notohara, Kenji................ 1768, 1769

Nour, Sherif G............................... 918

Novo, Manuela............................... 1952

Novotny, Alexander ....................... 664

Nowak, Jonathan.................... 752, 801

Nowakowski, Grzegorz S .......... 1408

Noy, Shawna ................................ 1723

Nozad, Sahar ......................... 241, 629

Nozaka, Hiroyuki .......................... 1573

Nozawa, Kumiko ......................... 1846

Nshimiyimana, Irenee ................... 223

Ntiamoah, Peter............................. 2062

Nucci, Marisa R .....1118, 1157, 1170, $1200,1239,1247,1248$

Nuciforo, Paolo ............................. 1813

Nunes, Cristiana B ......................... 711

Nuttall, David.................................. 437

Nwadei, Ifeoma.............................. 559

Nybakken, Grant ........................... 1430

Nyman, Mark ............................... 1557

O’Brien, John K ............................. 1190

O'Brien, Michael ............................ 804

O'Brien, Odharnaith ....................... 524

O'Brien, Shannon ....................... 1283

O'Connell, Fionnuala.......... 118, 126, $127,128,1574$

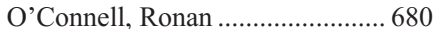

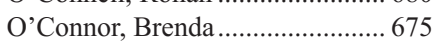

O’Connor, Kathleen L.................. 1926

O'Connor, William......................... 1926

O'Donnell, Kathryn ..................... 2080

O'Donnell, Patrick ......................... 525

O'Donoghue, Diarmuid .................. 680

O'Dorisio, Thomas......................... 568

O'Kane, Sara ................................... 1190

O'Keane, John Conor....... 2011, 2012

O'Leary, Gerard ............................ 1265

O'Leary, John........... 437, 1243, 1317,

1820,2034

O’Leary, John J .......1099, 1190, 1191,

1251

O'Leary, Matthew ....................... 1557

O'Malley, Dennis ............ 1440, 1517,

1518,1896

O'Neil, Maura .................................. 1631

O’Neill, Eric................................. 1779
O'Regan, Esther M …................... 1317 O'Riain, Ciaran ...... 1099, 1177, 2034 O'Shea, Robert.................. 1663, 1664

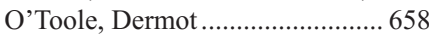
O'Toole, Sandra A............. 1292, 1293 O'Toole, Sharon......1099, 1190, 1191, 1243, 1820, 2034

Oak, Jean..................................... 1427

Oakes, Scott A....................................... 1761

Oakley, Fredrick D...................... 1192

Ober, Elisa..........................................111

Obstfeld, Amrom................................557

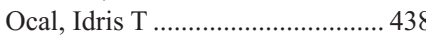

Ocana, Teresa .................................. 630

Ochiai, Atsushi ...................... 739, 832

Ochsenbein, Adrian....................... 1482

Oda, Yoshinao ................ 70, 97, 1868

Odze, Robert ....... 625, 626, 645, 646, $667,672,700,763,764,765$

Offerhaus, G Johan A..................... 811

Ogasawara, Sachiko ...................... 1223

Ogawa, Kumiko .............................. 683

Ogden, Angela.... 242, 243, 244, 2059

Ogino, Shuji .................................. 752

Oguejiofor, Kenneth...................... 2056

Ogura, Kanako ............................... 738

Ogurtsova, Aleksandra.................... 293

Oh, Danielle ................................... 1417

Oh, Hyeon Jeong.............................. 753

Oh, Young-Ha .............................. 1753

Ohbayashi, Chiho........................... 236

Ohe, Chisato............ 1005, 1045, 1048

Ohgami, Robert S... 1350, 1392, 1412

Ohike, Nobu..... 772, 826, 1738, 1772

Ohkawa, Shinichiro........................ 333

Oishi, Naoki ....................................... 597

Ojima, Hidenori ..................................... 1673

Okal, Ryan .................................... 1327

Okamoto, Aikou ............................... 1155

Okoye, Ekene ............................... 2037

Okten, Turhan ……………………..... 1

Olar, Adriana ..................................... 1172

Oldfield, Leslie...................... 317, 833

Oliva, Esther .............796, 1133, 1135 ,

$1195,1199,1201,1203,1227,1228$

Olivan, Mireia .................................. 894

Oliveira, Andre.................................... 37

Oliveira, Gabriel LP............. 866, 867

Oliveira, Rita SS .............. 1193, 1952

Oliver, Dwight ..................................... 1348

Olsen, Stephen ................................... 754

Olson, Kristin A ............ 586, 687, 755

Olson, Michelle ............................... 322

Olson, Nicholas.............................. 756

Olteanu, Horatiu..... 1333, 1446, 1447

Olvera, Narciso ............................. 1236

Omman, Reeba............................. 1991

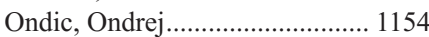

Ong, Clara .............................. 291, 292

Ong, Menchu................................. 1344

Onisko, Agnieszka .............. 479, 1238

Onozato, Maristela L...................... 320

Onuma, Hideyuki .............................. 735

Oprea-Ilies, Gabriela M ..... 260, 1964

Opyrchal, Mateusz ........................... 192

Orazi, Attilio .......... 1373, 1420, 1427 , $1455,1473,1486,1508,1522$

Ordi, Jaume ........... 1194, 1220, 1535,

1577

Ordulu, Zehra................................. 1195

OReilly, Catherine.
Orien, Jason.................................. 1016

Orloff, Mark ................................. 1642

Orlowski, Robert............... 1341, 1385

Orta, Lurmag....................................... 245

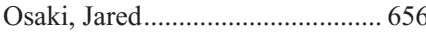

Osamura, Robert Y............................. 598

Osan, Remus ..................................... 304

Oshilaja, Olaronke O .................... 240

Oshima, Kiyoko ............... 1674, 1773

Osmond, Allison ............................... 757

Ostrovnaya, Irina............................... 847

Osunkoya, Adeboye O ........351, 393,

$864,865,873,898,902,918,926$, $1050,1062,1771,1811$

Otani, Kyoko................................ 1656

Otis, Christopher N ..................... 1802

Otsuka, Fumiyumi............................ 323

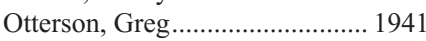

Otto, Joie ......................................... 21

Ou, Joyce J ..............1145, 1196, 1198

Ouansafi, Ihsane .......................... 1360

Ouseph, Madhu .......... 246, 758, 1378

Oussalah, Abderrahim.................. 1698

Ovcin, Emanuela...............................556

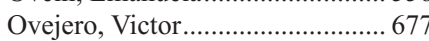

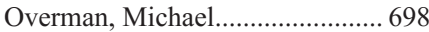

Owens, Scott R............................... 2008

Oxnard, Geoffrey R...................... 1922

Ozaki, Daisuke............................. 1869

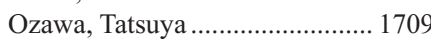

Ozerdem, Ugur.............. 125, 247, 248

Ozkaya, Neval................................ 1448

Ozluk, Yasemin ............................ 1307

Ozturk, Figen

Pac, Lincoln ........................ 759, 1646

Pace, Lydia ..................................... 223

Paddock, Christopher.................. 1541

Padera, Jr., Robert F ....................... 320

Padmanabhan, Vijayalakshmi ..... 358,

438,1787

Pagan, Carlos ............................... 1814

Page, Sylvain....................................... 336

Pai, Prathamesh.......................... 1288

Pai, Reetesh K..... 632, 673, 774, 825,

Pai, Rima.......................................... 912

Pai, Rish ............................................. 632

Pai, Rish K ....................... 1658, 1666

Pai, Trupti.................................... 249

Paietta, Elisabeth M .................... 1514

Paik, Paul K ................................ 1910

Paik, Seung Sam ................ 706, 1691

Paintal, Ajit ......................... 383, 424

Pairon, Jean-Claude ..................... 1878

Pak, Christine .................. 1144, 1379

Pal, Prodipto........... 1898, 1899, 1900,

Palacios, Jose ............................... 1197

Palanisamy, Nallasivam ... 1075, 1076

Palapattu, Ganesh................ 988, 1066

Palazzo, Juan P.............................. 676

Paliga, Aleksandra............................ 1449

Palisoul, Scott M.......................... 1770

Pallavi, Ranjita .............................. 1851

Palme, Carsten E........................... 1308

Palmerini, Emanuela ......................... 86

Paloppi, Isis................................. 1006

Palsana, Dhaval H....................... 1563

Paluru, Swetha ....... 1007, 1008, 1047

Pambuccian, Stefan.... 839, 855, 1975
Pambuccian, Stefan E ........ 362, 384 , $838,1261,1790,1991$

Pan, Chin-Chen ................ 938, 1009

Pan, Hongyang ............................... 976

Pan, Jincheng .............................. 1066

Pan, Wei ..................................... 1296

Pan, Zenggang................. 1352, 1450

Panarelli, Nicole.................. 659, 660

Panaretakis, Theocharis ............ 1497

Pandya, Chintan ........................... 965

Pane Foix, Maria ........................ 1010

Paner, Gladell P... 845, 852, 882, 885, $1011,1012,1632$

Pang, Judy C ....................... 422, 467

Pang, Xiaofan................................ 501

Pannu, Neesh............................... 1627

Pant, Mamta ................................ 1674

Pantanowitz, Liron.... 355, 464, 1557, 1560,1575

Pao, Andy ....................... 1815, 1872

Pao, Edward .................................. 456

Paolini, Michael ..................... 15, 1724

Papadopoulos, Nickolas...... 886, 887,

1004

Papanastasiou, Anastasios D ...... 250,

268,1250

Papanikolopoulos, Nikolaos ...... 1567

Papaxoinis, George .................... 1897

Papillon-Cavanagh, Simon ............ 54

Paquette, Cherie .........390, 1158, 1198

Paquin, Sarto C ............................ 343

Paradis, Valerie........ 1672, 1676, 1677

Parajuli, Shobha ......................... 1298

Parakh, Rugvedita ........................ 1013

Pardeshi, Vishakha..... 102, 251, 273, $1083,1084,1217$

Pareja, Fresia........................ 252, 253

Paresy, Marianne........................... 665

Parham, David........................... 1847

Parikh, Jignesh G .............. 778, 1675

Parikh, Melanie......................... 1030

Parikh, Rahul............................. 1017

Parimi, Vamsi .................. 1014, 1576

Parini, Vamsi ....................... 838, 839

Parisi, Joseph E ......................... 1724

Park, Christopher ... 1448, 1466, 1478

Park, Do Youn ............ 627, 760, 1753

Park, Donghyun ......................... 1743

Park, Emily .................... 1542, 2026

Park, Eun Su............................ 1015

Park, James S .................. 1629, 1654

Park, Jeongsoon ............................. 91

Park, Ji Young .............................. 1162

Park, Ji-Young.............................. 787

Park, Kay J ..............1166, 1185, 1219

Park, Kwang-Min....................... 1750

Park, Kyung ................................ 1901

Park, Minhee .................................... 705

Park, Sanghui .............................. 1015

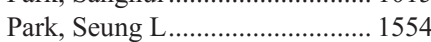

Park, Sung Hye .......................... 1407

Park, Sung-Hye ........................... 1710

Park, Sunyoung ........................... 1650

Park, Won Young ........................ 1015

Park, Woongyang ....................... 1743

Park, Yong Wook ....................... 1015

Parkash, Vinita .......... 545, 546, 1199 ,

Parker, Elizabeth U ...................... 560

Parker, Joel.................................... 1362

Parker, Lynette ..................... 410, 439 
Parks, Eric R

1432

Parra-Herran, Carlos .........1105, 1119, $1120,1141,1200$

Parra, Edwin........... 1931, 2080, 2081

Parsons, Lauren........................... 1617

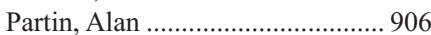

Parwani, Anil.......... 354, 1018, 1582, 2090

Paschke, Ralf................................... 458

Pasha, Shabana F ......................... 799

Pashankar, Farzana D.................. 1843

Pasic, Maria D................................ 896

Pastor, Francisco .......................... 1197

Pastorino, Ugo................... 1903, 1904

Patel, Jay L....................... 1339, 1979

Patel, Ketan ................................. 1982

Patel, Keyur P ....... 1374, 1388, 1389, 1390, 1391, 1424, 1464, 2003

Patel, Krishna ................................... 775

Patel, Natalie....... 654, 761, 813, 815,

1598

Patel, Nimesh R .......................... 1399

Patel, Nisha ……………........ 857, 858

Patel, Nupam.................... 1992, 1993

Patel, Rajiv .................................. 487

Patel, Robin................................. 1536

Patel, Roshan S ............................. 793

Patel, Sanjay................................. 1451

Patel, Sapna S.......... 762, 1397, 1452

Patel, Shraddha ............................ 2088

Patel, Snehal........... 1320, 1815, 1872

Patel, Tushar................................. 1686

Patel, Vatsal................................ 1299

Patel, Viren............................337, 468

Patil, Asawari ......... 1288, 1297, 1300

Patil, Dattatraya............................. 898

Patil, Deepa......... 748, 763, 764, 765,

$786,791,1648,1662,1663,1664$

Patterson, Andrew ......................... 495

Patterson, Kara ................. 1529, 1829

Patterson, Sara E .............. 1568, 1810

Pattillo, Chris ............................... 1344

Patton, Kurt T.................................. 39

Paueksakon, Paisit....................... 1592

Pauli, Chantal..... 23, 440, 1816, 2035

Paulk, Adina ................................. 1902

Paulson, Vera.................................. 599

Pavan, Nicola ................................ 1073

Pawel, Bruce R.............................. 980

Paweletz, Cloud .......................... 1922

Paxton, Christian N..................... 1339

Peabody, James .................... 955, 956

Pearlman, Rachel .......................... 788

Pease, Garrison ............................. 1994

Peckova, Kvetoslava ............ 81, 1010 ,

1027

Pederson, Elizabeth.......................... 91

Peevey, Joseph F ............................ 383

Pegtel, Dirk Michiel....................... 894

Pehlivanoglu, Burcin...................... 766

Peiper, Stephen C ......................... 2027

Peixoto, Renata ………………..... 1751

Pejchal, Martina ………………..... 1543

Peker, Deniz ................................ 1358

Pekmezci, Melike............... 1713, 1725

Pellegrinelli, Alessio ........... 591, 592,

1903

Pelletier, Daniel................................. 327

Pellicier, Jalidsa ............................. 474

Pellise, Maria ................................. 630

Pelosi, Giuseppe.

1903,1904
Pemmaraju, Manideepthi ............ 1192

Penault-Llorca, Frederique .......... 254

671

Peng, Bo ............................................... 1044

Peng, Limin ................... 200, 209, 238

Peng, Sui .......................................... 691

Peng, Xuan.................................... 192

Peng, Yan .................................... 1188

Peng, Yu-Ching ............................... 881

Pennington, Stephen R................. 1190

Penson, Alexander V ...................... 939

Pentecost, Brian T ........................ 1802

Pepperell, John R ......................... 1196

Pere, Helene ........... 1263, 1301, 1834

Pereda, Saray....................................... 677

Perencevich, Molly ........................ 801

Perez-Atayde, Antonio ...................... 36

Perez-Lopez, Raquel .......................... 1001

Perez-Ordonez, Bayardo ............. 1316

Perez, Adeline .............................. 1822

Periakaruppan, Ramayee............. 2063

Perincheri, Sudhir ....................... 1453

Perizzolo, Marco …………………..... 80

Perkins, Sherrie L... 1339, 1371, 1979

Perot, Gaelle...................................... 73

Perren, Aurel .... 585, 919, 1752, 1780

Perrino, Carmen M.............. 600, 1016

Perrone, Federica ......................... 1903

Perrone, Marie................................. 22

Perry, Anamarija .......................... 1454

Perry, Arie ............. 1713, 1715, 1725

Persad, Paul..................................... 1613

Pesci, Anna....................... 1201, 1227

Pessetto, Ziyan ............................... 143

Petaccia de Macedo, Mariana .... 2064

Peterson, Jason D ......................... 1787

Peterson, Kathryn.................................... 655

Peterson, LoAnn ............... 1467, 1473

Petersson, Fredrik .......................... 1279

Petimar, Joshua ............................ 1065

Petkiewicz, Stephanie .................... 525

Petrich, Adam.............................. 1334

Petrova-Drus, Kseniya ................. 1455

Pettaway, Curtis A............. 1022, 1023

Pettersson, Jonas .......................... 1092

Pettit, Kevin A................................... 526

Pfeifer, John D .................. 1601, 1914

Phaeton, Rebecca ............. 1127, 1246

Phan, Tien ................................... 1281

Pharaon, Majed ............................ 1215

Phelan, Sine....................... 2011, 2012

Philip, Philip .................................... 639

Phillips, Carrie L .......................... 2086

Phillips, Nancy J ............................ 767

Phillips, Sharon ............................. 1603

Piao, Jin............................................. 523

Piao, Jinhua ...................................... 767

Piao, Wenhua................................... 1414

Picard, Annabelle .......................... 1301

Picarsic, Jennifer .............................. 129

Picci, Piero ..........................56, 82, 86

Piccoli, Anthony L ..................... 1557

Pichardo, Janine ..... 1375, 1382, 1481

Picken, Maria M...... 855, 1014, 1071 ,

1576, 2036

Picquenot, Jean-Michel............... 1809

Pierrot, Sebastien .......................... 1834

Pierson, Tiffany …………………... 162

Pietanza, Maria ……………….... 1910

Pietsch, Torsten ........................... 1721

Pijuan, Lara ..

2024, 2077
Pike, John M ................................ 255

Pilichou, Kalliopi ......... 328, 329, 330

Pilkington, Loretto ....................... 1243

Pillai, Raju...................................... 1329

Pillappa, Raghavendra ............... 1675

Pimenta, Ruan CA....................... 1006

Pimiento, Jose M........................... 816

Pina-Oviedo, Sergio .......... 1456, 1457

Pinamonti, Maurizio ............. 497, 498

Pincus, Jennifer ..................... 203, 256

Pineda, Caryll................................ 1149

Pineda, Itzel................................... 1726

Ping, Zheng ......................... 257, 1458

Pinheiro, Celine.................................. 617

Pinheiro, Katherine ........................ 146

Pinnaduwage, Dushanthi............. 1967

Pintilie, Melania ............................ 1067

Pinto, Andre ........... 1202, 1206, 2019

Pinto, Carla .................................... 1203

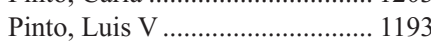

Pipas, J Marc ................................. 358

Pires-Luis, Ana S ............... 258, 1203

Pires, Andrea RC .......................... 2038

Piris, Miguel A .................. 1418, 1522

Piris, Miguel Angel ........................ 527

Pirog, Edyta C...................1138, 1179

Pirruccello, Elaina............. 1459, 1460

Pirruccello, Samuel J ....... 1366, 1530

Pisapia, David ...................... 23, 1816

Pisapia, Pasquale.................. 465, 808

Pischler, Carina ............................ 1721

Piscuoglio, Salvatore... 154, 160, 217 , $250,259,268,1250,1798$

Pisharodi, Latha R......................... 415

Pisters, Louis L .......................... 1061

Pistillo, Maria Pia.......................... 1861

Pitman, Martha................................ 442

Pittaluga, Stefania ............. 1376, 1483

Pittman, Meredith E.... 681, 768, 769,

Pivovarcikova, Kristyna... 1010, 1027

Pizem, Joze .................................... 498

Pizzi, Marco ................................. 1461

Pizzo, Donald P................................ 365

Pizzolitto, Stefano ............................111

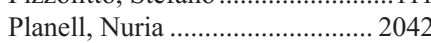

Plant, Pamela................................ 1033

Plesec, Thomas.............................. 1648

Pleskow, Douglas K ........................ 692

Plitas, George …............................ 276

Plotkin, Anna........................................ 1995

Plouhinec, Helene ....................... 1727

Plymate, Stephen............................ 976

Podduturi, Varsha ................................ 24

Podoll, Mirna B ................................. 1102

Point du Jour, Kimberly S............. 260

Poirier, John T ............................. 1910

Polanco, Carolina ............................. 770

Polano, Maurizio............................... 84

Polder, Julie G..................................... 587

Policarpio-Nicolas, Maria .............. 380

Poling, Justin................ 261, 681, 771

Polito, Humbert................ 1748, 1763

Pollack, Alan .................................. 969

Polydorides, Alexandros ................ 837

Polydorides, Alexandros D .......... 836

Pontes, Jose ..................................... 998

Ponzoni, Maurilio ......................... 1522

Pool, Kara-Lee .............................. 226

Popescu, Gabriel ............................. 215

Poppiti, Robert

228
Porcu, Pierluigi ............................. 1335

Poropatich, Kate................ 1204, 1205

Porter, Teresa C ............................ 1244

Portier, Bryce P.................... 151, 152

Portnoy, David .............................. 1614

Porwit, Anna ………………….... 1393

Posadas, Edwin M.......................... 878

Post, Kristin............................ 46, 873

Post, Robert.................................. 1575

Post, Steven................................... 1142

Pote, Nicolas .................... 1676, 1677

Poterucha, John ............................ 1658

Poulet, Bruno …………………....... 254

Poulik, Janet M ............................ 1848

Pouw, Roos E .................................. 811

Poveda, Andres ............................. 1197

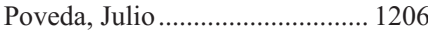

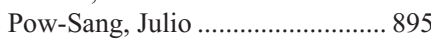

Powell, Isaac ......................... 890, 944

Powell, Simon ................................. 154

Powell, Suzanne Z ...................... 1553

Power, Derek..........................524, 733

Powers, Celeste N ..... 448, 471, 1990,

2004

Powers, Scott................................ 1431

Pozdnyakova, Olga ......... 1433, 1462 ,

$1512,1516,1584$

Prabhash, Kumar.......................... 1297

Pradhan, Anuja .............................. 1288

Pradhan, Dinesh ..........354, 457, 483, $859,1017,1018,1238$

Prajapati, Shyam ………………..... 554

Prakash, Gagan .............................. 995

Prakash, Nachiketh S .................... 969

Prakash, Sonam........................... 1513

Prall, Matt .................................... 420

Prandi, Davide.................................. 889

Prasad, Vinod K ............................... 668

Prat, Jaime ……………..................... 1195

Prayson, Richard ......................... 1702

Prazeres, Hugo ............................... 1193

Preffer, Frederic I ......................... 1974

Prendeville, Susan...................... 1019

Press, Richard ................................. 1449

Presta, Michael............................. 1470

Prichard, Jeffrey .................................. 409

Priemer, David ............................ 1020

Prieto, Victor G ... 488, 490, 505, 515, $523,530,534,535,540,2064$

Prihoda, Thomas ............................ 380

Pritchard, Colin ....................................... 1828

Pritt, Bobbi ................................... 1536

Priyanka, Priyanka ....................... 1374, 1388,

$1389,1390,1391$

Procop, Gary ................................. 2023

Profeta, Theresa L ........................... 234

Prokhorova, Ina............................ 1061

Prudkin, Ludmila ................................ 1813

Przybycin, Christopher G..... 905, 957

Ptashkin, Ryan .................. 1803, 1814

Puca, Loredana.................... 440, 1816

Puccio, Carmelo ............................ 1851

Puhalla, Shannon L ........................ 166

Puiggros, Anna............................... 2045

Pulliam, Lynn................................. 1953

Pulver, Tanya................................. 2001

Punia, Jyotinder N........................... 1399

Punnen, Sanoj ................................ 969

Punsoni, Michael................ 1728, 1729

Purgina, Bibianna............. 1271, 1311,

$1314,1315,2017$ 
Pusceddu, Sara …............................ 592 Pusztaszeri, Marc ....... 441, 468, 1302 Putman, Rachel K ....................... 1870 Putorak, Renn.................................. 381 Putra, Juan......................... 756, 1770 Pyo, Ju Yeon........................ 576, 2032

Pytel, Peter ..................................... 710

Qarmali, Morad.............................. 262

Qayyum, Sohail............................. 1463

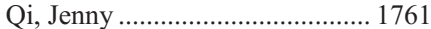

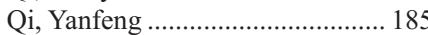

Qian, Xiaohua ……………... 402, 458

Qiu, Jihui J ....................................... 331

Qu, Hong ........................................ 1621

$\mathrm{Qu}$, Xiaoyu ....................................... 851

Quade, Bradley J...............1118, 1239

Quann, Kevin .............................. 1537

Quddus, M Ruhul................. 184, 390, 696, 975, 1088, 1145, 1146, 1150,

1198,1547

Quddus, Sana ............................... 1923

Querishi, Raheela........................ 2015

Quesada, Andres .... 1457, 1464, 1465

Quick, Charles... 561, 685, 907, 1142,

1200,2065

Quigley, Brian.......... 772, 826, 1738 , $1748,1764,1772,1784$

Quinones, Alexander K ................ 263

Quint, Wim......... 870, 871, 909, 1035

Quintana, Liza.............................. 1615

Quintana, Teresa.............................562

Quintanilla-Fend, Leticia ................ 1483

Quiroga-Garza, Gabriela............... 354

Qureishi, Hina N .... 1352, 1365, 1366

Qutub, Mohammed .......... 1736, 1737

Rabban, Joseph ......................... 1189

Rabban, Joseph T ...1149, 1151, 1165, 1435

Raca, Gordana................... 1434, 1504

Racanelli, Dominga......................... 84

Racean, Steliana F................ 527, 677

Racila, Emilian............................... 1905

Raciti, Patricia.............................. 1466

Radio, Stanley J ............................ 327

Radisky, Derek C ........ 119, 132, 133,

204

Radlinski, Brian C...................... 1021

Radpour, Ramin ........................... 1482

Radtke, Ina ................................... 1791

Rafeeq, Safia ........................ 698, 779

Raffals, Laura E .............................. 745

Raffeld, Mark ................... 1376, 1519

Rafla-Yuan, Eric ............................ 1021

Ragazzi, Moira ............................... 264

Raghunathan, Aditya .................... 1724

Rahemtulla, Amir ......................... 1282

Rahimi, Mahdi .................................. 675

Rahmani, Mahboubeh................. 1996

Rahn, Heidi ...................... 1467, 1468

Rahrig, Samantha.......................... 1805

Rai, Harpreet .................................... 321

Rai, Manoj.................................... 1024

Rai, Samarpit................................ 1073

Rais-Bahrami, Soroush ....... 884, 922,

992,1059

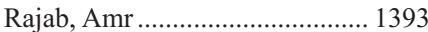

Rajoria, Gaurav ................... 983, 1081

Rakha, Emad ....... 160, 195, 196, 242,

243,250

Rakheja, Dinesh ......... 913, 914, 1348
Rakislova, Natalia ... 630, 1194, 1220, 1577,2042

Rakshit, Sagar ............................ 1583

Ram, Prahlad..................................... 534

Ramalingam, Preetha ...... 1125, 1207 ,

1209,1253

Raman, Jay D .............................. 1043

Ramandeep, Rattan ....................... 1083

Ramaswamy, Bhuvaneswari ...... 1829

Rambau, Peter ................................1111

Ramey, Jennifer K....................... 1434

Ramineni, Maheshwari ... 1906, 1936,

1937

Ramirez, Jose ................... 1577, 1907

Ramirez, Ricardo .......................... 847

Ramkissoon, Shakti H....... 611, 1696,

1699

Ramnefjell, Maria ........................ 1908

Ramon y Cajal, Santiago....... 49, 894 , 2073

Ramos, Benjamin...........................562

Ramos, Irma ................................... 2073

Rampal, Raajit K.......................... 1475

Ramsamooj, Rajendra .................. 1588

Ramsay, Jennifer ................................ 361

Ranade, Aditi................................. 996

Ranchal, Tamara............................. 1418

Rand, Jacob H .............................. 1838

Randev, Jabina ............................. 1469

Randhawa, Parmjeet .................... 1616

Randhawa, Simrath ...................... 1616

Randolph, Melissa L ........................... 2050

Rane, Swapnil .............................. 1300

Rangel-Filho, Artur .......... 1550, 1825

Rangel, Artur...................... 824, 1848

Rao, Amrita ................................ 1988

Rao, Arundhati ...... 95, 105, 171, 566,

1277,1984

Rao, Deepthi .................................. 265

Rao, Jianyu................. 456, 480, 1564

Rao, Nagesh P ............................. 456

Rao, Priya................. 951, 1022, 1023

Rao, Rema..........................440, 1816

Rao, Sam.......................... 1758, 1807

Rao, Sambasiva......... 577, 666, 1532, $1635,1636,1652$

Rao, Uma ……………………........ 52

Raoux, Delphine ............................ 493

Rapaport, Franck.......................... 1382

Raphael, Kalani................................ 1607

Ras, Aleksandra.................................. 2046

Rashid, Asif......... 623, 679, 698, 726,

750

Rashid, Tariq ............................... 1208

Rassidakis, George......................... 1497

Rasty, Golnar..................... 1200, 1219

Ratnam, Manohar........................ 1089

Raugnath, Mike............................ 2015

Raut, Prachi..................... 1122, 1924

Rautou, Pierre-Emmanuel........... 1672

Ravetti, Jean Louis........................ 1861

Ravi, Vinod ....................................... 61

Ravindranathan, Ajay.................. 2040

Ravishankar, Sanjita........... 490, 1209

Rawish, Kojo R ............................. 1210

Ray-Chaudhury, Abhik ............... 1726

Razavi, Pedram ............................. 270

Razmaria, Aria A............................. 852

Rea, Bryan..................................... 1508

Redd, Lucas................................... 1346

Redd, Robert.

1337
Reddy, Opal L

.. 1211, 1212

Reddy, Raveena............................... 231

Reddy, Vijaya B .............................. 169

Reddy, Vishnu .................... 1450, 1458

Reder, Nicholas P....... 266, 560, 1997

Redig, Amanda J .......................... 1909

Redline, Raymond W .................. 1842

Redman, Bruce............................. 1039

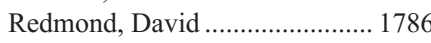

Reed, Jon A …………………….....505

Rehman, Aseeb .......... 271, 528, 1470

Reichard, R Ross.............................. 15

Reid, Michelle...................... 195, 196, $207,351,392,393,435,636$,

$772,773,826,1738,1748,1763$, 1764, 1765, 1766, 1767, 1771,

1772

Reid, Michelle D......... 242, 243, 244,

1784,2059

Reim, Daniel .................................. 664

Reindl, Bailey A... 179, 180, 237, 267

Reis-Filho, Jorge S...... 154, 160, 217 ,

$250,259,268,1250,1798,1823$

Reis, Katia.................................... 1952

Reis, Sabrina T ....................998, 1006

Reisenbichler, Emily S.................. 142

Reisian, Niloufar ................ 478, 2022

Reith, John D ..................... 536, 2047

Rekhtman, N .............................. 1894

Rekhtman, Natasha .......... 1880, 1910

Remotti, Helen ........... 694, 695, 1667

Ren, Bing ............. 643, 719, 774, 775

Ren, Fei...

$643,719,774,775$

Ren, Qinghu .................................. 1080

Rendi, Mara H...................... 266, 560

Renier, Corinne ................................ 456

Rennert, Hanna .................... 347, 1944

Renouf, Daniel ................... 794, 1751

Renwick, Neil .................................. 1856

Rescigno, Pasquale .......... 1001, 1002

Resetkova, Erika ................... 147, 287

Resnick, Murray............................ 1069

Resnick, Murray B ...... 246, 678, 696 , $758,809,823,827,974,1783$,

1793,1983

Retallack, Hanna EG..................... 121

Rets, Anton V .................... 1471, 1496

Reuter, VE..................................... 910

Reuter, Victor E... 846, 847, 939, 940, 1000, 1036, 1074, 1080, 1579

Revelo, Monica P....................... 1607

Revert, Jose B .............................. 1418

Rexer, Brent .................................... 155

Reyes-Mugica, Miguel..................... 11

Reyes, Carolina ..... 1136, 1200, 1213,

Reynolds, Alexandra 1222

Reynolds, Jordan P....... 436, 486, 957

Reynolds, Sam .............................. 562

Rezaee, Neda................................... 1054

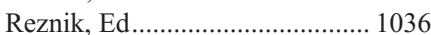

Rezvani, Katy................................... 912

Riad, Sara ....................................... 981

Riaz, Nadeem .................................. 154

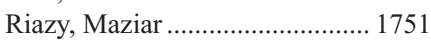

Ribalta, Teresa............................... 1844

Ricci, Andrew ........................ 140, 290

Riccio, Riccardo........................... 2090

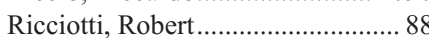

Ricciotti, Robert W ............................... 1671

Rice, Terri

1725
Richard, Hope T.......................... 1730

Richards, Elizabeth ....................... 776

Richards, Kristy ............................ 1362

Richmond, Abby M..................... 1839

Richter, James ..................... 182, 2001

Rickman, David ............................ 1816

Ricks-Santi, Luisel J ............ 187, 235

Rida, Padmashree CG ......... 195, 196, $242,243,244,304,2059$

Riddell, Robert H............... 675, 1540

Riedel, Elyn................................... 198

Rieger, Gregor...................... 662, 663

Riel, Stacy ...................................... 984

Riera Monroig, Josep .................. 1844

Riether, Carsten............................ 1482

Rigau, Marina ................................ 894

Righi, Alberto...... 56, 82, 86, 87, 569, 618

Riisnaes, Ruth .................. 1001, 1002

Riley, Elizabeth C ......................... 274

Riley, Jacquelyn .......................... 2023

Riley, Meghan P........................ 1472

Rimm, David L .................. 649, 1109

Rinaldo, Charles R ....................... 1545

Rinder, Henry ................................. 558

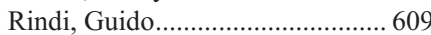

Ring, Kari..................................... 1214

Ritterhouse, Lauren L ................ 1248

Rivas, John ....................... 1663, 1664

Rivera, Christine .......... 151, 152, 791

Rizzo, Stefania ..... 328, 329, 330, 332

Ro, Jae Y ........................... 997, 1015

Roa, Benjamin B ........................ 505

Roa, Juan Carlos ... 1738, 1763, 1764,

Robadi, Ibrahim A............... 1215

Robboy, Stanley J........................... 1163

Roberts, Chris ............................... 1092

Roberts, Daniel E....... 51, 1626, 1678,

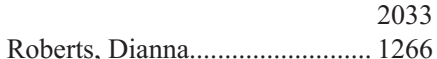

Roberts, Drucilla .................................. 1096

Roberts, Ian SD .......................... 1622

Roberts, James ................................... 879

Roberts, Jordan ........................... 2037

Robertson, Gordon .......................... 846

Robertson, Keith D ........................ 647

Robertson, Scott A .......................... 563

Robertson, Susan J....... 285, 862, 950

Robin, Adam ...................... 965, 1617

Robin, Yves-Marie ............................. 74

Robinson, Brian ......... 773, 777, 1964

Robinson, Brian D ........ 23, 440, 846,

1024, 1055, 1056, 1270, 1816, 1998,

2035

Robinson, Jeffrey ............................ 235

Robinson, Robert .......................... 1303

Rocha, Rafael M .............................................. 711

Rocha, Ruana M................... 866, 867

Rock, Jonathan ................................. 724

Roden, Anja C....... 1849, 1881, 1884,

1911

Rodic, Nemanja................. 511, 2074

Rodig, Scott............ 1144, 1366, 1379

Rodon, Jordi .................................. 1813

Rodriguez Canales, Jaime.......... 1044,

1937

Rodriguez-Canales, Jaime........... 212,

1931, 2080, 2081

Rodriguez-Pinilla, Maria.............. 1418

Rodriguez, Eva............................. 1844 
Rodriguez, Fausto ............. 1703, 1734

Rodriguez, Ingrid M............. 870, 871

Rodriguez, Maria DC.......... 883, 887,

1004,1054

Roehrl, Michael H....... 2, 3, 317, 637, $829,833,834,1826,1956$

Roemer, Margaretha GM .......... 1144

Roessler, Stephanie ..................... 1657

Rogers, Craig G ......... 917, 955, 956, 1074,1076

Rogers, Heesun J.... 1473, 1486, 1508

Rogers, Jessica G .......................... 586

Rogers, Thomas E............ 1609, 1621

Roh, Mee Sook............................ 1912

Roh, Michael H.................... 422, 467

Rojanapremsuk, Theera ............... 233

Rojas, Claudia ..................................... 416

Rojas, Claudia P.......... 375, 387, 778

Rojiani, Amyn ............................... 1711

Rokutan, Mariyo ………………... 1913

Roland, Joseph T ........................... 925

Roland, Robert ..................................543

Roldan, Belen..................................543

Roma, Andres A ....... 957, 1219, 1224

Romagosa, Cleofe .................... 49, 83

Roman, Kristin............................. 2057

Romanel, Alessandro ................... 1828

Romaschin, Alexander .................. 896

Romero, Eduardo ............................ 269

Romero, Ignacio............................ 1197

Romero, Maria ................................. 323

Romerocases, Gloria .................... 1041

Romo-Bucheli, David .................... 269

Ronaghy, Arash ............................. 1216

Roncella, Silvio............................ 1861

Ronchi, Susanna................................. 2066

Rong, Yuan.................................. 1730

Ronnett, Brigitte M........... 1219, 1226

Ronquillo, Nemencio .................... 779

Roobol, Monique ........................... 972

Rooney, Sydney .......................... 1303

Rooper, Lisa M ...... 1304, 1305, 1999

Roque, Ruben R ........................... 1193

Roquiz, Woodlyne............... 251, 1217

Rosa, Marilin................................... 272

Rosado, Flavia G.............. 1325, 1327

Rosario Quinones, Frances L.... 1474,

1475

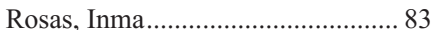

Rosca, Adriana …………………......... 203

Rose, Inga .......................................... 1797

Rosen, Daniel G ............................ 147

Rosen, Devin................................. 1599

Rosen, Seymour ..... 1137, 1599, 1615

Rosenbaum, Jason N.......... 601, 1914

Rosenbaum, Matthew W.............. 442

Rosenberg, Andrew E ................. 1312

Rosenberg, Jonathan E........ 846, 847,

939,1000

Rosenblatt, Mary P........................ 780

Rosenblum, Frida ............................ 367

Rosenblum, Marc ........................... 1448

Rosenblum, Norman ................... 1134

Roshal, Mikhail............... 1474, 1476,

1478,1954

Rosman, Martin............................... 205

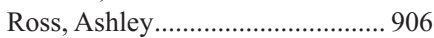

Ross, Dara S............... 268, 270, 1823
Ross, Jeffrey S............ 146, 241, 271 $300,528,611,629,716,758$,

$827,842,1025,1082,1123,1174$ $1260,1287,1470,1505,1699$ $1776,1785,1795,1860,1927$

Ross, Julia A................................. 1026

Rosser, Julie A..................... 443, 1477

Rossi, Esther .................................. 468

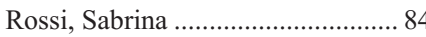

Rost, Sandra ...................................... 2067

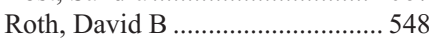

Roth, Rachel........................ 407, 788

Roth, Rachel M ............................... 724

Rothberg, Paul G............................ 303

Rotondo, Fabio............................ 1057

Rotterova, Pavla............... 1010, 1027

Rougemont, Anne-Laure............. 1840

Rousseau, Audrey .......................... 1727

Roussel, Helene............................. 1263

Routbort, Mark...... 1374, 1388, 1389 1390, 1391, 1464, 1526, 2003,

2058

Rovin, Brad ...................................... 1591

Rovira, Carlota .............................. 1844

Rowan, Daniel........... 644, 689, 1674 ,

1773

Rowe, Neal............................................. 1610

Rowe, Steve P ............................. 927

Roy Chowdhuri, Sinchita.... 426, 444, $445,1957,2003$

Roy, Angshumoy ......................... 1399

Roy, Somak ..... 354, 859, 1017, 1018

1722

Roychoudhury, Sudarshana......... 1218

Royer, Robert.............................. 1087

Royston, Daniel................. 1375, 1478

Ruano, Ana........................ 1479, 1480

Rubin, Brian P.......... 66, 67, 68, 250

1250,1823

Rubin, Charles.............................. 1434

Rubin, Mark ........ 23, 440, 889, 1002 , $1024,1056,1786,1816,1828$,

2035

Rubinstein, Mara R ....................... 712

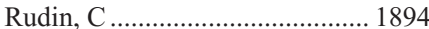

Rudin, Charles M.......................... 1910

Rudomina, Dorota............. 1580, 1581

Rudzinski, Erin R............................. 85

Rueter, Jens ....................................... 1810

Ruiz-Cordero, Roberto................... 602

Rumery, Mathew ............................ 1966

Ruminy, Philippe............................... 38

Runza, Letterio............................... 1865

Ruo, Leyo......................................... 361

Rusch, V ................................ 1894

Rush, Natalia................................. 1679

Russell, Maria ................................. 773

Russo, Mariano .............................. 1246

Rustin, Jeannette G ........................ 325

Ruterbusch, Julie J ......................... 251

Rutgers, Joanne KL............ 767, 1219

Rutter, Sara................................. 124

Ruttle, Carmel ............................... 2034

Ruzinova, Marianna B ..... 1330, 1413

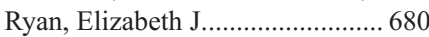

Ryan, Philip....................... 2011, 2012

Rysgaard, Carolyn............... 496, 2000

Ryzhova, Elena .............................. 895

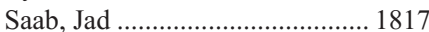

Saade, Rami E................................ 1266

Saadeldine, Mothana.
Sabel, Michael

Sabido, Eduard

Sabir, Sharjeel

Sabourin, Jean-Christophe .... 38, 665 ,

Sacher, Adrian .............................. 1922

Sacks, Wendy ................................. 359

Saco, Adela ............. 1220, 1577, 1907

Saddiqui, Saaduddin ...................... 575

Sadimin, Evita T ..... 994, 1028, 1029 ,

1030

Sadot, Eran.................................... 719

Sadow, Peter M ........... 441, 458, 579,

Sadowska, Justyna ................... 2041

Sae-Ow, Wichit ............................ 1481

Saeed-Vafa, Daryoush.................... 272

Saeed, Faisal ...................................... 781

Saeed, Omer AM.............................. 1031

Saegusa-Beecroft, Emi.................. 2068

Saenz, Barbara ............................. 1130

Safran, Howard ................................ 827

Sagasta, Amaia ................................ 1220

Saglam, Ozlen ................... 1221, 1240

Sahai, Anand V ................................ 343

Sahin, Aysegul A .......... 106, 150, 307

Sahnane, Nora …………………... 1500

Sahoo, Sunati .................................... 233

Sahu, Divya........................................... 1032

Said, Ian ........................................ 1229

Said, Jonathan ................................... 480

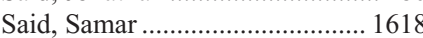

Saiji, Essia....................................... 1840

Saiki, Mitiko ......... 1006

Sainati, Laura ............................... 1461

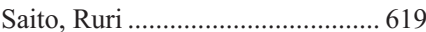

Sajed, Dipti ......................... 1306, 1821

Saka, Burcu.... 772, 1748, 1763, 1764

Sakaguchi, Asumi ........................... 738

Sakakibara, Rie ............................. 1915

Sakamoto, Michiie ....................... 1673

Sakamoto, Reid ................................ 381

Sakamoto, Yoshihiro ......... 1660, 1661

Sakr, Sharif....................... 1083, 1084

Sakr, Wael ....................................... 944

Sakr, Wael A......................... 890, 903

Saksena, Annapurna ...................... 1525

Salahuddin, Sadia.......................... 1362

Salama, Mohamad E....... 1339, 1353, $1451,1471,1496,1558,1584$

2006

Salama, Sherine................................. 2001

Salaria, Safia N............................ 2002

Salaverria, Itziar............................ 1483

Salazar, Paulo A ........................... 2062

Saleeb, Rola ........... 1033, 1034, 1057

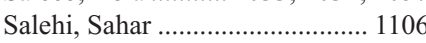

Salem, Fadi ...................................... 454

Salem, Nagla .............. 273, 1090, 1217

Sali, Aakash....................................... 995

Salido, Marta …………….............. 2045

Salim, Alaa A ................................ 2003

Salimian, Kevan ............................. 1916

Salipante, Stephen J ...................... 1394

Salmon, Lauren E.......................... 1491

Salomao, Diva R ………………..... 1951

Saltel, Frederic .............................. 1672

Saltzman, John R …….................... 801

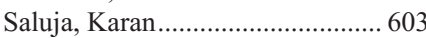

Salvatore, Steven........ 23, 1589, 1619
Salvi, Sandra ............................... 1861

Samarapungavan, Dilip............... 1605

Samayoa, Luis.............. 144, 219, 286

Samedi, Von G ............................... 562

Samghabadi, Peyman................... 1966

Samimi, Setareh ........................... 1751

Samolczk, Julia .................................. 383

Samore, Wesley.............................. 782

Samowitz, Wade S ............... 621, 728

Sampathkumar, Ashwin .............. 2068

Samuelson, Megan I.....................1117

Samulski, T Danielle............ 564, 699,

1222

Sanada, Sakiko............................ 1223

Sanati, Souzan...... 188, 189, 190, 306

Sanchez, Ariadna........................... 630

Sanchez, Carlos............................. 2089

Sanchez, Diego F ........ 870, 871, 909 ,

Sanchez, Ignacio ........................ 2024

Sanchez, Rodrigo ............................ 421

Sanchez, Sandra ................ 1370, 1437

Sanchez, Sergio ............................... 1917

Sandell, Rosalind ............................. 2069

Sander, Chris ................................. 1036

Sanders, Mary Ann......................... 274

Sanders, Melinda E .......................... 155

Sanders, Victoria ........................... 1810

Sanford, Eric M........................... 1123

Sanger, Warren G ......................... 1530

Sangoi, Ankur R..... 1189, 1224, 1435

Sanguino Ramirez, Angela M... 1818 ,

Sanmann, Jennifer N.................. 1530

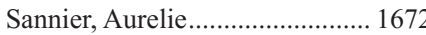

Sannigrahi, Soma ........................... 1811

SantaCruz, Karen ............................... 1724

Santagada, Eugene ......................... 1216

Santagata, Sandro........................... 1696

Santi, Raffaella............................. 1003

Santin, Alessandro D..........1109, 1123

Santoro, Luisa ............................... 1461

Santos Cavalcanti, Marcela......... 718 ,

$783,784,785$

Santos, Liliana................................. 258

Sapino, Anna .................................. 217,669

Sapp, Heidi...................................... 732

Saqi, Anjali..................................... 460

Saqib, Naveed U ……………….... 313

Sarafan-Vasseur, Nasrin ................. 665

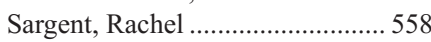

Sari, Sule ................................... 1307

Sarkar, Fazlul ................................. 639, 1825

Sarkar, Gobinda ........................... 1725

Sarkaria, Inderpal S..................... 1776

Sarmiento, Juan....... 772, 1763, 1764,

$1765,1766,1767$

Sarungbam, J................................ 910

Sarungbam, Judy .............. 1036, 1080

Sasano, Hironobu ..................... 26, 583

Sassa, Naoto .............. 405, 1063, 1064

Sassoon, Daniel J .................. 941, 942

Sastri, Jayant ................................. 1701

Satgunaseelan, Laveniya .............. 1308

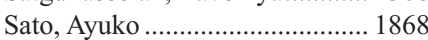

Sato, Daisuke .................................... 1760

Sato, Shun ........................................ 1037

Satoskar, Anjali .............................. 1620

Satou, Akira

Saullo, Jennifer L ............................... 668

Sauter, Jennifer L ............... 446, 1225 
Savage, Amanda.......................... 437 Savage, Erica....... 763, 764, 765, 786,

Sawabe, Motoji .................................. 333

Saxe, Debra F................................ 1639

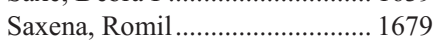

Saxena, Rovin ................................ 447

Sayada, Chalom ............................. 2083

Sayeed, Hadi ................................... 1399

Sayeed, Sadia ..................... 448, 2004

Sazonova, Olga .............................. 336

Sbaraglia, Marta ................. 82, 86, 87

Sboner, Andrea ............ 23, 889, 1024, $1786,1816,1828,2035$

Scala-Bertola, Julien .................. 1698

Scalia, Carla R ………………...... 2066

Scandura, Glenda ......................... 1038

Scarborough, John.............................. 88

Scardino, Peter ............................... 863

Schaberg, Kurt ................................ 449

Schadendorf, Dirk ......................... 1721

Schaefer, Inga-Marie................. 39, 89

Schaefer, Rachel............................ 1078

Schaeffer, David F ..... 694, 794, 1751

Schaeffer, Edward M ........... 849, 906

Schafernak, Kristian T ................ 1520

Schaffer, Andras ................................ 501

Schafroth, Christian ........................ 663

Schalper, Kurt .............................. 1109

Schantz, Daniel ............................... 380

Schatz-Siemers, Nina ..................... 275

Scheier, Benjamin ............................ 1039

Schembri Wismayer, David ....... 1951

Scherpelz, Kathryn....................... 1309

Scherpereel, Arnaud ...................... 1878

Scherr, Douglas............................. 1024

Schiano, Thomas............................... 1683

Schiavo Lena, Marco ................... 1841

Schieda, Nicola ..................... 862, 950

Schiffman, Marc............................ 2035

Schildhaus, Hans-Ulrich .............. 1918

Schilling, Bastian ........................... 1721

Schirmacher, Peter ....................... 1657

Schittenhelm, Jens................. 545, 546

Schlachter, Simon ……………........ 672

Schlegel, Peter N.......................... 1998

Schlesinger, Kathie ......................... 394

Schmechel, Stephen C ................. 374

Schmidt, Janine .............................. 1483

Schmidt, Robert L .............. 115, 1955

Schmidt, Ryan ................................ 1433

Schmidt, Yao ................................ 1346

Schmitt, Alessandra...................... 1964

Schmitt, Anja M .......................... 1972

Schmitt, Sarah ................................ 143

Schmitz, Katja ................................. 1918

Schneider, Kelli........................... 1445

Schneider, Thomas........................ 1578

Schnitt, Stuart............................... 160

Schoedel, Karen .................................. 52

Schoenberg, Mark ………………... 856

Schofield, Kevin..................... 342, 353

Scholz, Simone …….................... 1721

Schoolmeester, John..........1120, 1156, $1166,1200,1226$

Schowinsky, Jeffrey T........ 443, 1477

Schreckenberger, Paul................. 1533

Schuh, Andre ............................... 1393

Schultenover, Stephen J ................... 7

Schultheis, Anne M..... 217, 250, 939,
Schultz, Daniel.......... 107, 186, 1085

1217

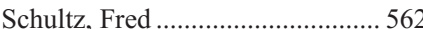

Schultz, Nikolaus ............................. 795

Schurch, Christian......................... 1482

Schwalbe, Michael L...................... 90

Schwartz, Lauren ..... 557, 1120, 1200

Schwartz, Lauren E....................... 991

Schwartz, Mary R ...... 352, 427, 1970

Schwartz, Robert A ...................... 1950

Schweitzer, Karen .......................... 1326

Schwertfeger, Kathryn L............... 182

Sciallis, Andrew ……………….......... 1108

Sclafani, Francesco G .................... 680

Scognamiglio, Theresa.... 1270, 1998 ,

2018

Scolyer, Richard A ............ 1308, 1721

Scott, David W ............................... 1408

Scott, Richard................................. 908

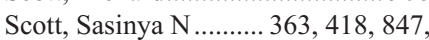

$939,1000,1827$

Scotti, Mauro..................................... 591

Sebastian, Christopher .................... 1948

Sebastiano, Christopher ....... 276, 277

See, William................................. 1565

Seethala, Raja R ......... 464, 510, 604, $605,1271,1310,1313,1315$

1316,1749

Segal, Jeremy ...................................... 710

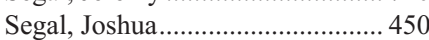

Segal, Neil..................................... 718

Segura, Ana M ...................................... 313

Segura, Sheila E .............................. 234

Seheult, Jansen ............................... 1545

Seibel, Eric J .................................. 2070

Seidman, Michael A................ 12, 321

Seiler, Christian A ................................. 670

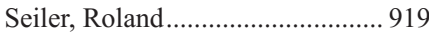

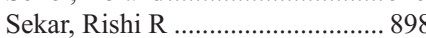

Sekeres, Mikkael A ...................... 1432

Seki, Atsuko ....................................... 333

Sekine, Yasuo ................................. 1869

Seko, Shuko ....................................... 405

Seldenrijk, Cornelis A .................... 811

Selegean, Sorin ............................... 732

Self, Craig W................................... 2002

Selig, Martin .................................. 2041

Selinger, Christina I ......... 1292, 1293

Sell, Evan W ................................. 182

Selove, William............................. 1484

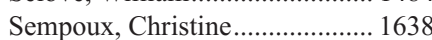

Sen, Filiz ……………………...... 1475

Sensi, Elisa ......................................... 570

Seo, An Na ....................................... 787

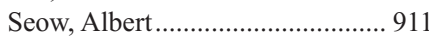

Sequeiros, Tamara ........................... 894

Serdy, Kate M .............. 166, 278, 279

Serra, Eduard............................... 49, 83

Serra, Stefano ..................................... 747

Serrano, Laia .............. 893, 2045, 2055

Serrano, Sergio............ 947, 948, 960, 2024, 2045, 2055, 2077

Seshan, Surya ................... 1589, 1619

Sesok-Pizzini, Deborah.................. 548

Sessa, Fausto ............................. $585,609,1500$

Sethi, Aisha .................. 788, 789, 790

Sethi, Sanjeev..............

1590

Sethi, Seema.

791

Setia, Namrata............... 632, 633, 792

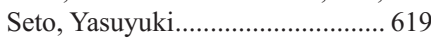

Settanni, Giulio .............................. 1903
Settelmeyer, Teagan .................... 1830 Settembre, Elizabeth D ................. 1559 Seven, Ipek E ................................ 1766 Seviour, Elena G ............................... 534 Seward, David................................... 91 Sezak, Murat .................................... 766 Sfakianos, John P .............. 877, 1000 Shabaik, Ahmed .................. 478, 1049 Shabani-Rad, Meer-Taher ......... 1361,

1469

Shabihkhani, Maryam

... 25

Shackelford, Rodney

1819

Shackelford, Rodney E

Shafizadeh, N ............................... 810, 820

Shah, Akeesha ........... 605, 1310, 1315

Shah, Alpa ................................... 1040

Shah, Alpa B …………………......... 853

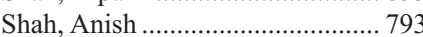

Shah, Girish V ................................ 954

Shah, Kabeer ......................... 500, 529

Shah, Rajal .......... 949, 977, 978, 979, $1041,1042,1058$

Shah, Ronak ……………............. 1000

Shah, Sejal S ........... 686, 1643, 1645, 2005

Shah, Tanmay ..................... 451, 1043

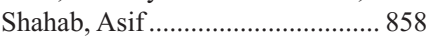

Shahi, Maryam..................... 182, 452

Shahlaie, Kiarash ................586, 1712

Shahsafaei, Ali ........... 94, 1493, 1494

Shaib, Walid ....................................... 773

Shaikh, Fyza........................................ 775

Shailendra, Giri ............................ 1083

Shakil, Fouzia ………………….... 1851

Shalin, Sara .................................... 528

Shalin, Sara C................................. 552

Shamash, Jonathan .............................. 1038

Shanes, Elisheva ………………... 1994

Shankaran, Veena ................................. 759

Shannon, Kristen M .................... 1203

Shanti, Rabie

92,1819

Shao, Haipeng ................... 1400, 1528

Shao, Hiapeng ............................... 1328

Shao, Lina ........................................ 1438

Shao, Tiffany …………………......... 280

Shareef, Hasanain ............................ 963

Sharif, Asma...................................... 793

Sharifzad, Hamidreza............. 2, 3, 317

Sharma, Meenal .......................... 1642

Sharma, Rajni..... 261, 293, 934, 1054

Sharma, Sachein.............................. 1519

Sharma, Sameer ........................... 1095

Sharma, Shree G …………........... 957

Sharp, Adam.................... 1001, 1002

Sharp, Linda .................................. 1243

Shaw, Andrea J.............................. 1605

Shaw, Gene ................................ 1451

Shaw, Kenna ... 199, 642, 1044, 1104,

1788,2049

Shazly, Sherif ................................. 102

Sheahan, Kieran ........ 658, 680, 1762,

2011,2012

Sheahan, Patrick.......................... 1265

Sheehan, Christine E... 271, 300, 629, $716,717,1082,1860$

Sheffield, Brandon S ........... 281, 794,

1751,1919

Sheikh-Fayyaz, Silvat ........ 165, 1380

Sheikh, Umer ...................... 453, 1621

Sheils, Orla........................ 1820, 2034

Sheldon, Kathryn E..................... 1246

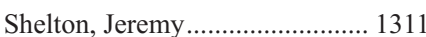

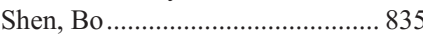

Shen, Dejun................. 168, 257, 1458

Shen, Jia ........................................... 79

Shen, Lin .......................................... 713

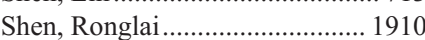

Shen, Rulong.................................... 401

Shen, Shih-Che .............................. 297

Shen, Steven S ............................... 997

Shen, Tiansheng ........... 282, 283, 284

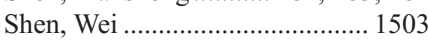

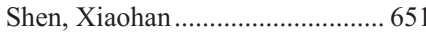

Shen, Xuxia.................................... 1101

Sheng, Dong.................................. 1403

Sheng, Shijie ................................... 903

Sheng, Weiqi ................. 651, 751, 805

Sherer, Carol R............................... 1414

Sherman, William ........................ 2008

Shetty, Prakash ............................. 1701

Shetty, Shashirekha ...... 151, 152, 791

Shi, Chanjuan............... 469, 674, 818

Shi, Dongping ............ 890, 944, 1090

Shi, Min.................. 1338, 1442, 1782

Shi, Qiuying .................................. 1280

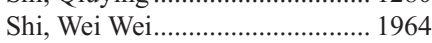

Shi, Yu......................................... 1681

Shia, Jinru ... 693, 718, 719, 783, 784, $785,795,1775,1801,1814$

Shibahara, Junji................ 1660, 1661

Shibahara, Yukiko ............................. 26

Shidham, Vinod B .................. 398, 639

Shields, Anthony .............................. 639

Shih, Angela R .......... 796, 797, 1201,

1227,1920

Shilo, Konstantin........................... 1941

Shim, Hyemin ............................... 2048

Shimada, Hideaki ......................... 1869

Shimizu, David ...........463, 656, 1114,

1244

Shimizu, Michio............... 1738, 1772

Shimosegawa, Tooru ..................... 1769

Shin, Eunah .........................576, 2032

Shin, Hyeong C ............................ 1742

Shin, Nari ...............................627, 760

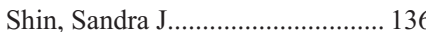

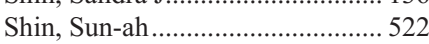

Shinkai, Masato................ 1845, 1846

Shinoda, Hiroko ...................................... 656

Shintaku, Itsushi P............ 1211, 1212

Shipe, Tracy ........................................ 9

Shipp, Margaret A ........................... 1144, 1379

Shireen, Rizmee ............................. 1191

Shirsat, Hemlata ............................. 1921

Shiue, Yow-Ling ……………….... 690

Shivdasani, Priyanka............. 599, 752

Shojaei, Hadi................................. 1842

Sholl, Lynette M..... 403, 1870, 1887, $1900,1909,1922,1932$

Shon, Wonwoo ... 489, 504, 536, 2047

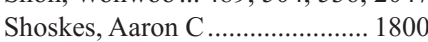

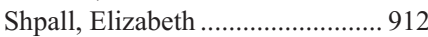

Shrestha, Rupendra ...................... 452

Shrestha, Swati.................................. 79

Shrock, Devin L ........................... 798

Shroff, Stuti G.... 699, 731, 807, 1639

Shuch, Brian.................................... 841

Shulman, Lawrence N..................... 223

Shun, Chia-Tung .............................. 723

Shvartsbeyn, Marianna ………..... 2006

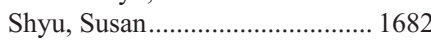

Si, Qiusheng .................................... 454 
Sibley, Richard K

Sibony, Mathilde ............................... 1062

Sicotto, Hugues ............................. 1725

Sidawy, Mary K …............................ 346

Siddiki, Hassan .............................. 799

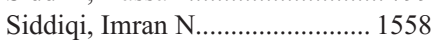

Siddiqui, Iram ................................ 1774

Siddiqui, Javed ............................. 1066

Siddiqui, Momin T...... 349, 350, 429, $453,580,1283,1964,1989$

Siddon, Alexa ..................................521

Sidoli, Simone...................... 96, 2087

Siegal, Gene P..... 257, 282, 283, 284,

$\begin{array}{rr} & 1808 \\ \text { Siegelbaum, Robert } & 1580\end{array}$

Siegele, Bradford .............. 1477, 1485

Siek, Lidia E...................................... 361

Siever, Jodi E ............................... 1281

Sievert, Margarit ………………..... 878

Sigal, Samuel .................... 1629, 1654

Sigel, Carlie................ 783, 784, 1740

Sigel, Keith …...................... 783, 784

Signaevski, Max............................ 2020

Sikora, Andrew G....................... 1284

Silva, Annacarolina ........... 1227, 1228

Silva, Elvio.................................... 1124

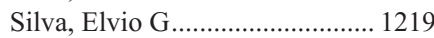

Silva, Fernanda ............................ 1126

Silva, Mike................................... 1779

Silva, Milton JDBE ....................... 1641

Silva, Monique F......................... 2038

Silveira, Armandina ...................... 1193

Silverman, Jan.............................. 2076

Silverman, Jan F............... 1818, 1933

Silvero, Arturo...................................... 871

Sim, Jongmin .......................................... 101

Sim, Jordan .................................... 285

Simi, Lisa .......................................... 56

Simko, Jeff .......................... 993, 1060

Simko, Jeffrey P........................... 929

Simon, Mercedes.............. 2024, 2045

Simonson, Paul D......................... 2070

Simper, Novae................................ 968

Singal, Amit ......................... 653, 725

Singh, Arun ……………………...... 79

Singh, Charanjeet.............................. 1161

Singh, Hardeep.............................. 1948

Singh, Harsharan ............................ 1612

Singh, Kamaljeet....... 184, 218, 1150,

1983

Singh, Manmeet ............................... 2007

Singh, Manpreet............................. 813

Singh, Nanda............................... 1214

Singh, Naveena …............. 1152, 1229

Singh, Rajendra.............................. 502

Singh, Rajesh .... 444, 534, 721, 1464, $1705,1957,2003,2058$

Singhal, Rashi L........................... 1439

Singhb, Baljit ................................ 185

Singhi, Aatur ..... 775, 825, 996, 1749 ,

1775,1776

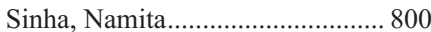

Sinnwell, Jason P ........................... 305

Siqueira, Deborah P .................... 2038

Sirca, Kanishka ................................ 951

Sircar, Kanishka ....... 602, 1022, 1044

Sirintrapun, S Joseph ...... 1036, 1080, $1579,1580,1581$

Sirintrapun, Sahussapont J .......... 885,

939, 940, 1000

Sirintrapun, SJ 910
Sirlin, Claude ………………….... 2078

Sirohi, Deepika ......... 933, 986, 1012, 1030, 1045, 1046, 1047, 1048

Siroy, Alan E ................................... 530

Sisto, Andrea ....................................... 82

Siziopikou, Kalliopi P ................... 203

Skacel, Marek ………………...... 1810

Skala, Stephanie L................ 531, 988

Skalova, Alena .................. 1271, 1282

Skehan, Sinead .............................. 1820

Skinner, Kristin ..................... 138, 303

Skinner, Mary.................................... 91

Sklar, Jeffrey L....................................... 1843

Slager, Susan L............................. 1363

Slater, Shahrzad ................ 1145, 1230

Sloan, Emily A ... 27, 587, 1181, 1231

Sloan, Sonia ................................... 437

Slodkowska, Elzbieta......... 167, 1087, 1097, 1098, 2071

Slovak, Marilyn L ........................ 1432

Smith, Catherine ………... 1514, 1515

Smith, Emily J.............................. 1933

Smith, Geoffrey....... 435, 1578, 1609 ,

1989, 2029

Smith, Kelly ........................ 334, 1946

Smith, Kenneth M........................ 1951

Smith, Kerrington D............................ 358

Smith, Kristen ................................... 565

Smith, Lauren B ............... 1438, 2008

Smith, Lynette M ......................... 1352

Smith, R Neal.............................. 1585

Smith, Steven ...... 30, 448, 471, 1045 ,

1045,
1048

Smith, Theresa …………………..... 455

Smola, Brian ................................... 422

Smolkin, Mark E.......................... 1158

Smyrk, Thomas C ....... 715, 745, 803 ,

Smyth, Lilian............................... 268

Snape, Katie ................................. 1048

Snarskis, Connor ............................ 1071

Snipes, George J............................ 1812

Snir, Olivia L................... 1232, 1843

Snyder, Dana R ............................... 1942

Snyder, Michael P ........................ 2052

Soares, Maria Fernanda .............. 1622

Sobottka, Bettina............................. 298

Soderquist, Craig.......................... 1486

Sohani, Aliyah R .... 1325, 1337, 1974

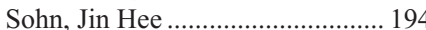

Soike, Thomas...................................... 550

Sojitra, Payal .................... 1487, 1923

Sokol, Lena ............................. 662, 663

Solanki, Shraddha ................ 362, 397

Solcia, Enrico .................................... 609

Solit, David B.... 268, 847, 939, 1000 ,

Sollier, Elodie....................................... 456

Solomon, David ................................ 657

Solomon, David A............................ 1233

Solomon, Isaac H .......................... 1544

Solomon, James ..... 1049, 1731, 2009

Soloway, Roger ........................... 1687

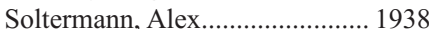

Somoza, Rosa................................. 2073

Song, Jinming .................... 1400, 1528

Song, Joo Y ....................... 1329, 1423

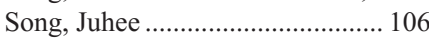

Song, Sangyong .......................... 2048

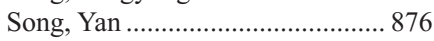

Soni, Abha................................... 1554
Sonmez, Ceyda ................................ 200

Sonzogni, Angelica ........... 1903, 1904

Soo, Chia........................................... 79

Soonawalla, Zahir ........................ 1779

Soong, Thing Rinda ............ 801, 1234

Soria, Jean-Charles ...................... 1822

Soslow, Robert .......1139, 1140, 1153, $1186,1227,1235,1236,1250$

1823

Soubrane, Olivier .............. 1672, 1677

Souers, Rhona J.......... 438, 461, 1331

Soukiazian, Nyrie.................. 295, 296

Soundar, Esther ............................. 1167

Soundararajan, Suganthi ............. 2088

Sovoda, Rebecca .......................... 1605

Sowalsky, Adam G...................... 1078

Spalding, M John ................................. 6

Spaulding, Reed ............ 144, 219, 286

Spears, Grant ................................ 1849

Spence, J David.............................. 2025

Spence, Janice M ............. 1397, 1398

Sperga, Maris .................... 1010, 1027

Spiess, Philippe E..... 895, 1022, 1023

Spillane, Cathy D ... 1099, 1820, 2034

Spotlow, Vanessa.......................... 2046

Springer, Simeon........ 886, 887, 1004

Spruill, Laura S ……………….... 1208

Spyropoulos, Demetri .................. 1811

Sreedhar, Hari ………................... 2063

Sreekumar, Arun ............................. 171

Srigley, John R .............................. 1074

Sripakdeevong, Parin ....................... 2052

Srivastava, Amitabh .... 742, 752, 801,

1757

Srivastava, Sucheta ...114, 1183, 1237

Srougi, Miguel ................... 998, 1006

Staats, Paul.................... 410, 439, 470

Stacey, Xavier ............................... 1833

Stachler, Matthew D .......................... 801

Stadler, Nadine ............................... 1721

Stadler, Zsofia ....................... 719, 795

Staerkel, Gregg ... 371, 426, 444, 445,

1457

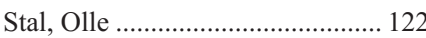

Staley, Charles................................. 773

Staley, Christopher .......................... 773

Stallworth, Christina ....................... 957

Stamelos, Evangelos ..................... 1579

Stanek, Jerzy ……………………...... 16

Starikov, Roman S ..................... 1835

Stark, Alejandro ............................... 812

Starosta, Tim .................................. 315

Starostik, Petr.................................... 647

Stas, Marguerite ............................... 492

Stashek, Kristen .......... 699, 731, 807

1639

Statz, Cara ........................ 1568, 1810

Steele, Lucinda.................................... 433

Stefansson, Ingunn .......................... 110

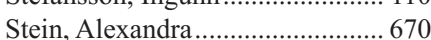

Stein, Tracy A............ 606, 1122, 1924

Steinhoff, Margaret ............... 218, 390

Steinmetz, Jamie ......................... 1925

Stemmer, Melissa ............................ 432

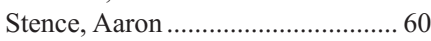

Stephanopoulos, Gregory............. 1332

Stephen, David ............................... 2007

Stephens, Craig ............................. 1092

Stephens, Julie A ……................... 1148
Stephens, Philip J........ 146, 241, 271 , $528,611,842,1025,1123,1260$, $1287,1505,1699,1785,1795$

1927

Stetler-Stevenson, Maryalice .... 1507,

1519

Stevens, Keith .............................. 1050

Stevens, Todd M .......................... 922

Steward, David............................... 473

Stewart, Colin JR .......................... 1170

Stewart, Douglas Allan .... 1361, 1417

Stewart, John M ... 425, 444, 445, 726

Stewart, Rachel ........................... 1926

Steyerberg, Ewout ............................ 972

Stickland, Kyle..............................1118

Stieglitz, Elliot ............................... 1513

Stiller, Mathias ............................... 1721

Stingo, Francesco ............................ 1873

Stocchi, Luca.................................... 786

Stock, Wendy …………………........ 1504

Stockdale, Colleen .........................1117

Stoff, Benjamin K ........................... 559

Stohr, Bradley ..................... 929, 2040

Stoler, Mark H..........1158, 1181, 1182

Stolnicu, Simona ............................ 287

Stone, John H ............................... 1336

Stoos, Catherine ………....... 288, 2010

Stopa, Edward G .............. 1728, 1729

Stopinskijs, Aleksijs ....................... 543

Storer, Chad ............................................... 601

Stout, Clarke …………………….... 29

Stram, Michelle... 28, 457, 483, 1238 ,

Stranahan, Alec ............... 1466

Stratman, Curtis .......................... 1557

Straub, Shana ...................... 802, 1051

Street, Leslie ......................................... 1361

Streutker, Catherine J ...................... 669

Stricker, Hans ................ 955, 956, 985

Strickland, Kyle C...... 458, 613, 1239

Strickland, Sarah ..........................1119

Strohecker, Ingrid........................... 2073

Strosberg, Carolina........................ 1240

Stuart, Joshua M............................. 846

Stueck, Ashley E ……………….... 1683

Sturgis, Charles D ........ 240, 377, 438

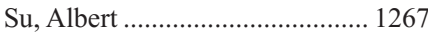

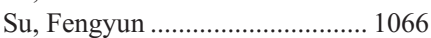

Su, Nan................... 2026, 2051, 2085

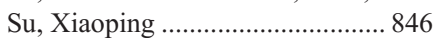

Suarez, Adrian A ................ 724, 1148

Suarez, Maria J............................. 1734

Suarez, Yvelisse .................. 708, 831

Subramaniyam, Shivakumar ...... 1901

Suciu, Christopher........................... 1545

Sucker, Antje ............................... 1721

Sugawara, Yumi ............................... 26

Sugiura, Yoshiya ..................................... 93

Suh, Ja Hee........................................ 459

Suh, James............ 146, 241, 271, 528,

$611,842,1025,1123,1260,1287$, $1505,1699,1785,1795,1927$

Suh, Yeon-Lim .............................. 1714

Sukhadia, Purvil ............................... 57

Sukhai, Mahadeo...................................... 1393

Sukhanova, Madina....................... 1504

Sukov, William R ......... 325, 500, 536,

Sule, Norbert ............................ 1264

Suleiman, Sarah ................................ 1620

Sullivan, Brian ............................ 2035 
Sullivan, Megan ............................... 256

Sullivan, Peggy ………………...... 226

Suman, Vera J.............. 229, 230, 305

Sumiyoshi, Shinji ......................... 1864

Sun, Heather................................. 1144

Sun, Hongxia.................. 141, 197, 289

Sun, Hongzhe.............................. 1542

Sun, James.......................... 758, 1123

Sun, Jun.......................................... 620

Sun, Li............................. 713, 876

Sun, Shihua ......................................... 976

Sun, Tianlin ...................................... 468

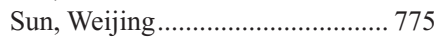

Sun, Xiangjie......................................... 805

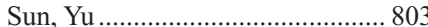

Sun, Yue ............................. 804, 1052

Sun, Zhuoxin................................ 1514

Sunday, Mary E..................................... 513

Sundell, Brian ................................ 672

Sundi, Deb...................................... 849

Sundling, Kaitlin ................................ 607

Sundram, Uma .................................. 547

Sung, Anthony D.............................. 668

Sung, C James... 184, 390, 696, 1088, $1145,1146,1196,1198,1547$

Sung, Chang Ohk ........................... 459

Sung, Ji Youn ................................. 1015

Sung, Simon..................................... 460

Sung, Sun Hee............................... 1015

Sung, Yun-Shao....... 33, 47, 58, 1316,

1836

Sunkara, Jaya ................................. 394

Sunol, Mariona............................... 1844

Suresh, Raagini ............................. 639

Suriawinata, Arief A........... 756, 1770

Surti, Urvashi .............................. 1436

Suryavanshi, Moushumi ............ 1045

Suster, Saul................ 81, 1010, 1027

Sutherland, Russel............... 897, 1794

Svajdler, Marian ............................ 1027

Svrcek, Magali ................................. 664

Swager, Anne-Fre............................ 672

Swan, Niall.... 658, 1762, 2011, 2012

Swanson, Benjamin J .................. 2072

Sweeney, Brenda J ........................ 431

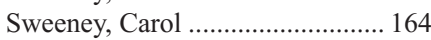

Sweeney, Melissa ............................. 470

Sweeney, Robert T ........................ 211

Swerdlow, Steven H.......... 558, 1402, $1414,1415,1436,1463$

Swick, Brian.......................... 496, 526

Swinarski, David........................... 460

Swisher, Shannon ............................ 307

Swisher, Stephen G ...................... 1906

Swisshelm, Karen ........................ 1485

Sy, Keiyan ……………………....... 1995

Sybenga, Amelia ............................ 566

Sykes, Don ................................... 2060

Sykulski, Maciej ............................ 935

Symbol, Brittany ….......................... 290

Syngal, Sapna................................. 1775

Szablewski, Vanessa..................... 1273

Szankasi, Philippe .......................... 1503

Szentgyorgyi, Eva ......................... 747

Szeverenyi, Nicklaus.................... 2078

Szporn, Arnold H ........................... 454

Szymanski, James ........................... 174

Tabatabai, Z Laura ........................... 461

Tabernero, Josep............................. 746

Taccioli, Cristian .......................... 1351

Tacha, David .......... 1049, 1360, 2091
Tachibana, Tomoyoshi ................... 583

Tackett, Alan ................................ 1142

Tada, Toyohiro ………………….... 532

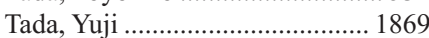

Tadros, Talaat S................ 1964, 1965

Tadros, Yousef............................... 1041

Tafe, Laura J............ 148, 1787, 1857,

1928,1929

Tagawa, Masatoshi....................... 1869

Tagawa, Scott T............................ 1024

Taggart, Melissa W ..... 623, 679, 698,

Tahara, Ippei............................. 597

Taheri, Diana....... 883, 886, 887, 934, $1004,1053,1054$

Tajiri, Takuma ....................... 772, 826

Takahashi, Hiroyuki ..................... 1037

Takahashi, Satoru ........................... 1063

Takano, Masato …........................... 236

Takeda, Maiko................................ 236

Takei, Yusuke ............................... 1406

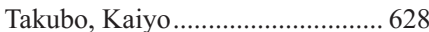

Talhouk, Aline..........1131, 1132, 1140

Taljaard, Monica ........................... 1200

Tallman, Martin S ....................... 1514

Talmon, Geoffrey A........................ 888

Tam, Wayne............... 817, 1420, 1508

Tamagnini, Ione .......................... 264

Tamboli, Pheroze ... 1022, 1023, 1044

Tamborini, Elena........................... 1903

Tamimi, Rulla M........................... 117

Tan, Benjamin Yongcheng ... 291, 292

Tan, Brent......................... 1966, 1973

Tan, Char-Loo …………………...... 533

Tan, Cong .............................. 751, 805

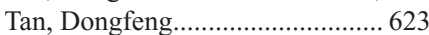

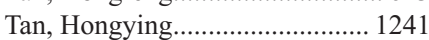

Tan, Kay See ................................. 1880

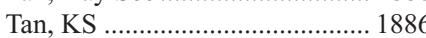

Tan, Leonard HC........................... 1489

Tan, Lingyun .................................... 484

Tan, Puay Hoon............. 104, 176, 177

Tan, Puay-Hoon .................... 291, 292

Tan, Wei ........................................ 1264

Tan, Yaohong ......... 1312, 1437, 1490

Tanaka, Kuniaki ............................ 1063

Tanaka, Maki................................... 227

Tanaka, Mio ..................... 1845, 1846

Tanaka, Miwa.................................. 98

Tanaka, Yukichi................. 1845, 1846

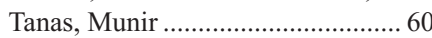

Taneja, Krishan ............................... 223

Tang, Guilin .......... 1377, 1395, 1396, $1416,1424,1473,1492,1509$

1525

Tang, Ping ................... 138, 295, 296

Tang, Shangguo................................ 361

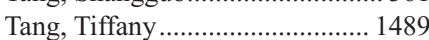

Tang, Weihua....................................... 1491

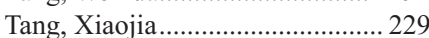

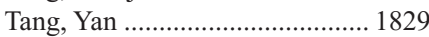

Tang, Zhaoyi ................................. 1716

Tang, Zhenya..................... 1395, 1424

Tangella, Krishnarao ....................... 215

Tanguay, Jeffrey ..................................... 1863

Taniguchi, Hiroyuki ...................... 1867

Tantravahi, Umadevi..................... 1196

Tao, Xiang ........................... 462, 482

Tapia, Coya ……………………...... 307

Taplin, Mary-Ellen ......................... 1078

Taraboanta, Catalin ...................... 1242
Tarakanova, Vera.......................... 1446

Tardif, Jean-Claude ……………... 2025

Tarrell, Robert F ………………..... 999

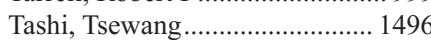

Tashkandi, Hammad........................ 843

Tasian, Sarah ..................................... 1515

Taskin, Orhun C ........................... 1307

Tateyama, Hisashi ............................532

Taube, Janis....... 293, 806, 856, 1053,

1267

Tauchi-Nishi, Pamela.......... 381, 463 ,

735,1114

Taute, Lindsay ..................................... 14

Tavakkoli, Montreh..................... 1478

Tavassoli, Fattaneh A ............. 247, 248

Tavassoli, Peyman........... 1024, 1055, 1056, 1998

Tawedrous, Eriny ............... 1033, 1057

Tawfik, Ossama............ 143, 255, 265

Tay, Jessica................................... 1292

Taylor, Barry S .................... 847, 939

Taylor, Martin .............................. 1821

Taylor, Norman ..................................578

Team, Anatomic Pathology ......... 1971

Tebit, Emaculate V .......................... 162

Teixeira, Manuel R....................... 1203

Teixido, Cristina..................................... 49

Tejaswi, Sooraj.................................. 687

Tempero, Margaret A .................... 1755

Tempier, Ariane .............................. 1273

Temprana-Salvador, Jordi ............ 894, 2073

ten Kate, Fiebo J ............................ 811

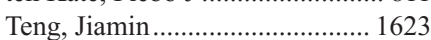

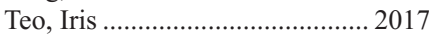

Teot, Lisa A ..................................... 458

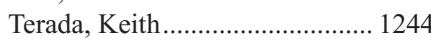

Teresi, Peggy .................................. 1797

Terracciano, Luigi M...................... 1657

Terribas, Ernest ................................... 83

Terrier, Philippe.................................. 74

Terris, Benoit............ 671, 1738, 1772

Tesdahl, Brennan.......................... 1303

Tessier-Cloutier, Basile ............... 1153

Tetzlaff, Michael T...... 488, 490, 515, $523,530,534,535,540,1705$,

2064

Tewari, Anupama ……………...... 1354

Tewari, Prerna ........ 1243, 1251, 1317

Thai, Anne ....................................... 687

Thakker, Sushama ............................... 1549

Thakral, Beenu ...... 1374, 1388, 1389,

$1390,1391,1492$

Thalmann, George N..................... 919

Thangamani, Muthukumar.......... 1619

Thapa, Prabin ................................. 999

Theis, Jason D ............ 163, 319, 1590

Theisen, Brian ................................ 464

Theodosakis, Nicholas ................. 2074

Thiele, Jurgen ..................................... 1410

Thiene, Gaetano ... 328, 329, 330, 332

Thike, Aye Aye ..... 104, 177, 291, 292

Thomas, Cody ………………..... 1812

Thomas, Dafydd............................ 1039

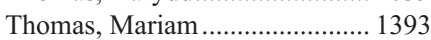

Thomas, Martha ............................ 1181

Thompson, Carlie K............... 121, 122

Thompson, Elizabeth ........... 293, 806

Thompson, Erika J ....................... 1422

Thompson, Ian ................................. 993

Thompson, Kevin J ........................ 229
Thompson, Lester DR....... 608, 1258, $1271,1272,1316$

Thompson, R Houston ................... 999

Thomsen, Matthew B.................... 1992

Thomson, Thomas............ 1281, 1282

Thorson, John A .................. 962, 1731

Thota, Prashanthi N ...................... 791

Thrall, Michael J ........................... 1553

Thung, Irene ................................... 1049

Tian, Shaozhou........ 1880, 1910, 2075

Tian, Wei ......... 977, 979, 1041, 1042,

1058

Tian, Xin ...................................... 1507

Tian, Xuejun................94, 1493, 1494

Tibert, Babylonia ........................... 1106

Tibshirani, Robert ........................... 1431

Tickoo, Satish K.......... 847, 939, 940 $1000,1036,1080$

Tickoo, SK ……………................ 910

Tiemeny, Placede ………...................... 1874

Tihan, Tarik …………....... 1713, 1725

Tijssen, Jan G............................................. 811

Timon, Conrad ............................. 1317

Timothy, Greiner C ...................... 1365

Ting, David T ........... 821, 1747, 1821

Ting, Kang ........................................ 79

Tirode, Franck ................................... 73

Tissier, Frederique.......................... 593

Tjendra, Youley ................... 1370, 1495

Tjionas, George A .......................... 943

Toetsch, Martin ................................556

Toganel, Cornelia ................................... 287

Toledo, Gemma............................ 1135

Tolle, Brian.................................... 2008

Tomaszewicz, Keith.... 509, 885, 982 ,

1523

Tomaszewski, John ..................... 1280

Tomita, Yasuhiko ............................. 72

Tomlins, Scott ……............................. 1048

Tomlins, Scott A........ 889, 988, 1045,

1278,1805

Tomoka, Tamiwe......................... 1362

Tondon, Rashmi ........ 699, 807, 1298 ,

1639

Toner, Mary

.. 1317

Torbenson, Michael...................... 686, 1643, $1644,1645,1657,1658,1665$ $1666,1684,1685$

Torkko, Kathleen C ............... 443, 954

Torlakovic, Emina............ 2020, 2021

Tormey, Chris..................................... 521

Torne, Aureli ................................. 1220

Torre, Matthew.................................. 1732

Torregrossa, Liborio....................... 570

Torrence, Dianne .................................536

Torres-Cabala, Carlos A...... 488, 490, $523,530,535,540$

Torres, Alfredo ............................. 1495

Torres, Javiera ................... 1738, 1772

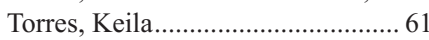

Torres, Maria L................. 1818, 2076

Torrez, Mary...................................... 791

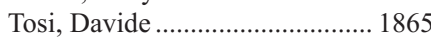

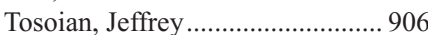

Toth, Laura N ...................... 1928, 1929

Toukatly, Mirna ............................ 1624

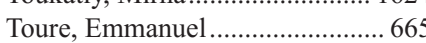

Toussaint, M Andrew ............ 902, 918

Townsley, Danielle M ...... 1357, 1405

Toydemir, Reha M ....................... 1471

Tozbikian, Gary............ 159, 172, 263 
Tracht, Jessica …......................... 1059

Traer, Elie..................................... 1449

Trahan, Sylvain ............................. 336

Train, Laura J ...................................... 324

Tran-Thanh, Danh ............................ 343

Tran, Henry ........................................ 1847

Tran, Nam .................................... 1149

Tran, Truc........................................ 1043

Tranchida, Paul ........................... 1090

Tranesh, Ghassan ......................... 1686

Travis, W......................... 1886, 1894

Travis, William............................ 1880

Travis, William D......................... 1910

Traynor, Oscar................................. 658

Treaba, Diana O .......................... 1378

Treacy, Ann ...................... 2011, 2012

Trejo Bittar, Humberto.... 1890, 1891 ,

1892,1930

Treloar, Murray ......................... 1582

Tretiakova, Maria S..... 851, 855, 880 , $993,1013,1060$

Treurniet, Kilian M ....................... 900

Trevino, Karen Elizabeth ... 920, 1020

Trevisan, Giorgia.......................... 1229

Triboulet, Melanie........................... 456

Tripp, Sheryl R................ 1353, 1496

Trochesset, Denise ....................... 1261

Trock, Bruce.................................... 906

Troester, Melissa ........................... 193

Troncone, Giancarlo.............. 465, 808

Troncoso, Patricia ........................ 1061

Trotter, Marin J .............................. 1572

Troxell, Megan............... 5, 294, 1833

Troyer, Dean........................ 993, 1060

Trpkov, Kiril..............546, 1062, 1074

True, Lawrence ........ 993, 1060, 1997

Truong, Luan D ............................... 997

Truong, Tra.................................... 2013

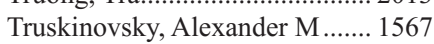

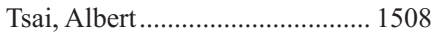

Tsai, Jen-Wei ..................................... 53

Tsao, Ming-Sound ........................ 1863

Tschen, Jaime ................................... 505

Tse, Darren .................................... 2017

Tse, Julie Y ................................... 1546

Tse, Ka Yu ...................................... 1147

Tseng, Hsian-Rong ......................... 878

Tseng, Li-Hui ................................ 1854

Tseng, Ling-Min ........................... 134

Tseng, Yun-An................................ 809

Tsesmetzis, Nikolaos....................... 1497

Tsiatis, Athanasios C............ 868, 989

Tsividakis, George ...................... 1579

Tsongalis, Gregory J ....... 1770, 1787, $1810,1928,2046$

Tsongalis, Peter D ....................... 1787

Tsuchida, Takashi .......................... 532

Tsuijikawa, Hanako........................... 1673

Tsuji, Ichiro ...................................... 26

Tsujimura, Tohru .......................... 1868

Tsuzuki, Toyonori ..... 405, 881, 1011, 1063,1064

$\mathrm{Tu}$, Xiaoyu .....................................1112

Tubbs, Raymond .................... 151, 152

Tucker, Tracy ..................................... 44

Tull, Jamie......................................... 99

Tuluc, Madalina ………………......... 1271

Tumminello, Katherine ................ 1498

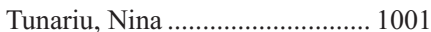

Tung, Rebecca................................ 1987

Tung, Spencer

1716
Turakhia, Samir................ 1326, 1499

Turbat-Herrera, Elba A..... 1587, 1623

Turmel-Roy, Justine ....................... 336

Turner, Bradley .... 138, 205, 295, 296

Turner, Jerrold R ……………….... 824

Turpin, Sara B ................................. 1193

Tuschl, Thomas ............................ 1856

Tuthill, Mark ................................. 1961

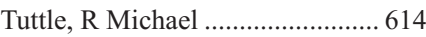

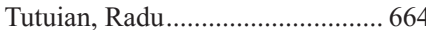

Twaddell, William S......... 1682, 1693

Tworek, Joseph A ......................... 1331

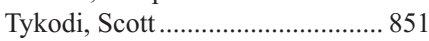

Tylee, Tracy S ............................... 374

Tyler, Janice ........................ 466, 2014

Tyner, Angela .................................. 793

Tyrrell, Jillian ................................ 2091

Tyryshkin, Kathrin ............. 1417, 1856

Tzalis, Vasilis ................................... 206

Tzankov, Alexander ...................... 1522

Ubago, Julianne M ........................ 203

Uccella, Silvia .................... 585, 1500

Uchiyama, Tomoko ......................... 236

Ud Din, Nasir ....................... 100, 1271

Udager, Aaron .......... 988, 1039, 1065, $1066,1268,1313$

Uehara, Tsuyoshi............................ 1768

Ueki, Toshiharu ............................. 1769

Ueng, Shir-Hwa ................... 173, 297

Ueno, Naoto T................................. 149

Ueshima, Chiyuki ......................... 1406

Ugolini, Clara...................................... 571

Uguz, Alper ................................. 1692

Ulamec, Monika........................... 1010

Ulbright, Thomas M.... 920, 921, 941, $962,1008,1020,1031$

Umbricht, Chris B ............................ 205

Umetsu, S.................................... 810

Unger, Pamela .................................. 857, 1216

Upadhyay, Swikrity....................... 943

Uppal, Guldeep ………………..... 2027

Upton, Melissa P.......................... 2070

Uraizee, Imran................................. 335

Uram-Tuculescu, Cora ................... 448

Urbauer, Diana ............................... 1164

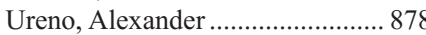

Uruga, Hironori............................. 1920

Ussene, Esperanca......................... 1535

Vachharajani, Neeta ..................... 1640

Vacirca, Davide ............................. 1865

Vafaii, Pardis ...................... 1444, 1501

Vaickus, Max...................... 804, 1052

Vainer, Ben.................................... 1269

Vairamuthu, Hemamalini ............. 1781

Vajpeyi, Rajkumar............................ 747

Vakar-Lopez, Funda ............... 855, 993,

1013,1060

Vakiani, Efsevia .......... 718, 719, 783, $784,785,795,1801,1814$

Valasek, Mark ………………........ 2078

Valdez, Janet ................................. 1357

Valdivia-Fernandez, Rodrigo .......903

Valea, Fidel A................................. 1121

Valencia-Cedillo, Raquel .............. 850

Valent, Peter ...................................... 1371

Valente, Alfredo ...................... 99, 432

Valente, Marialuisa ..... 328, 329, 330,

Valentino, Alessandro ...... 1279, 1861

Valenzuela, Gerardo.......................... 543

Valeri, Barbara

1903,1904
Vallangeon, Bethany .................... 1502

Valle, Maria.................................... 1423

Valluru, Neelima ........................... 1790

Valo, Isabelle......................................... 74

Valori, Laura ……………………..... 84

Valverde, Claudia ............................... 49

van de Nes, Johannes ................... 1721

Van Deerlin, Vivianna M ............ 1262

Van Dellen, Melissa .......................... 29

van den Oord, Joost......................... 492

Van Der Kwast, Theo ....................... 900

Van der Kwast, Theodorus H ...... 971, $972,1019,1067$

van der Wel, Myrtle J..................... 811

Van Dyke Darkow, Grant............ 1724

Van Dyke, Alison L.......... 1738, 1772

van Leenders, Geert .............. 971, 972

Van Ness, Michael ........................ 1503

van Overeem Hansen,

Thomas... 1269

van Rhijn, Bas WG ........................ 900

van Slambrouck, Charles ............ 1504

Van Vrancken, Michael ................... 720

van Zante, Annemieke .................. 431

van't Veer, Laura J ......................... 122

VanBuren, John ................................ 526

VandenBussche, Christopher J...... 11, $345,369,370,886,1004$

Vander Heide, Richard S............. 1556

Vanderboom, Patrick...................... 489

Vanderhyden, Barbara........1119, 1141

VanDeusen, Matthew A................ 1933

Vanel, Daniel....................................... 86

Vanoli, Alessandro ............................. 609

Varadan, Vinay ............................... 158

Varadhachary, Gauri R......... 726, 750

Varambally, Sooryanarayana....... 1358

Vardiman, James W..................... 1434

Vardouniotis, Alexandra.................... 594

Varesano, Serena ........................... 1861

Varga, Zsuzsanna .................. 250, 298

Varier, Indu...................................... 1284

Varma, Hemant ............................ 2079

Varma, Sushama.................... 156, 211

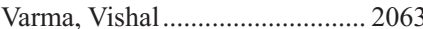

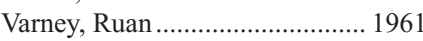

Varshney, Neha ……………….... 1625

Vasef, Mohammad ........................ 1340

Vasiliadis, Theodore.............. 331, 337

Vassella, Erik.................... 1752, 1780

Vats, Pankaj.................................... 1066

Vaucher, Jonathan .......................... 336

Vavinskaya, Vera ............................. 2078

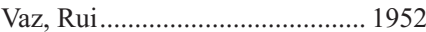

Vazquez, Ivonne ...... 960, 2024, 2045

2055,2077

Vega, Francisco..... 1370, 1425, 1437, 1490,1495

Veigl, Martina …............................. 822

Veillon, Diana ……………………. 1344

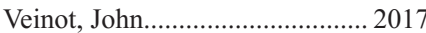

Vela, Deborah...................................... 313

Velasco, Valerie .................................... 73

Velazquez, Elsa F ............... 909, 1035

Velcheti, Vamsidhar ...................... 1583

Velez Torres, Jaylou ................................ 610

Vella, Jo.......................................... 1229

Velosa, Claudia .............................. 1280

Veltri, Robert W …t................. 511

Venkataraman, Girish................... 1328

Venkatraman, Lakshmi ............... 1777
Vennam, Sujay .............................. 211

Vera, Francisco............................... 1197

Verardino, Gustavo C.................. 2038

Vergilio, Jo-Anne ........ 146, 241, 271 , $528,611,842,1025,1123,1260$

$1287,1505,1699,1785,1795$

1927

Verhoef, Esther............................... 972

Verstovsek, Srdan.......................... 1508

Veyliotti, Andrew ………………..... 420

Viale, Agnes ........................ 847, 2062

Viana, Nayara..................................... 998

Vidhun, Ramapriya ......................... 234

Vieites, Begona ............................. 1197

Vielh, Philippe.............................. 1822

Vierkant, Robert A ................ 132, 133

Vierkoetter, Koah R ..................... 1244

Vigliar, Elena........................... 465, 808

Vignaud, Jean-Michel .................. 1698

Vigneswaran, Wickii ..................... 1850

Vigouroux, Charlene .................... 1698

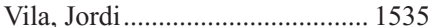

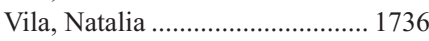

Vilaro', Marta.................................... 1813

Villa, Celina .................................. 1506

Villalobos, Pamela .......... 1931, 2080, 2081

Villanueva-Siles, Esperanza..........216

Villanueva, Alberto ........................... 49

Villaseca, Miguel A......... 1738, 1763,

1772

Vincent-Salomon, Anne ............... 1798

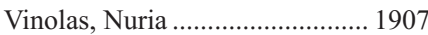

Vinters, Harry V ............................ 1716

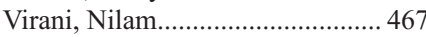

Virmani, Renu ...................... 322, 323

Vishal, Sagar J................................... 1778

Visscher, Daniel ............................. 133

Visscher, Daniel W...... 119, 132, 204 $229,230,251,305,2005$

Visser, Mike .................................. 811

Viswanatha, David S.................... 1386

Viswanathan, Akila ..................... 1144

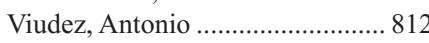

Vivancos, Ana ............................... 1813

Vivero, Marina ......... 458, 1870, 1898 ,

Vlad, George 1992

Vogel, Ulrich ................................... 2082

Vogelstein, Bert.........886, 887, 1004

1053

Vohra, Manjiv................................... 299

Vohra, Poonam ..... 213, 214, 299, 388

Voidazan, Septimiu ........................ 287

Voisey, Kim .................................... 2013

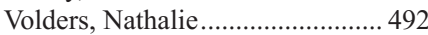

Volel, Vladimir H .......................... 2015

Volinia, Stefano ............................. 1351

Voltaggio, Lysandra ..... 681, 769, 771

Volyanskyy, Konstantin ................. 308

Volz, Marcus .................................. 1277

von Buchwald, Christian.............. 1269

von Bueren, Erico .......................... 2089

Vora, Moiz............................ 92, 1819

Voronel, Olga ..................300, 611, 1082

Vos, Jeffrey....................... 1325, 1327

Voznesensky, Olga ....................... 1078

Vrana, Julie ............... 319, 1590, 1611

Vrana, Julie A................................. 163

Vranic, Semir ................................ 1797

Vrotsos, Elena ........... 301, 1206, 2016 
Vujanic, Gordan 1841

Vuong, Huy ....... .597

Vural, Cigdem 1184

Vyas, Monika ...... 645, 646, 761, 813,

814,815

Vytlacil, Christopher ................... 1933

Wachtel, Mitchell S....................... 1068

Wachter, David L ......................... 1086

Wack, Katy..................................... 1582

Wagner, Jay ..................................... 558

Wagner, Susanne ............................ 1874

Waha, Andreas ............................. 1721

Wajapeyee, Narendra ..................... 815

Wake, Laura .......................................... 1366

Wake, Laura M............................. 1507

Wakely, Paul E ............................... 407

Wala, Samantha J ............. 1033, 1057

Walavalkar, Vighnesh...... 1593, 1626,

Walch, Axel ...................... 670

Wald, Abigail I ................................. 1722

Walecha, Prerna .............................. 249

Wales, Cameron .............................. 2083

Waljee, Akbar................................. 725

Walker, Kimberly L...... 95, 105, 1277

Walker, Rachel .............................. 816

Wallace, Michael................ 199, 2049

Wallace, Zachary S ..................... 1336

Walsh, Joanna C........................... 757

Walsh, Kyle M ............................. 1725

Walsh, Michael.......... 335, 793, 2036, 2063

Walsh, Paul......................... 118, 1574

Walter, Otto ........................................... 659

Walther, Zenta .............................. 1843

Walts, Ann E ...... 123, 302, 359, 573, $1869,1872,1934,1935$

Wampfler, Jason A........................ 1855

Wands, Jack................................... 1789

Waner, Emily E .................................. 374

Wang, Charlotte I ......................... 1757

Wang, Chi..................................... 1926

Wang, Chichung ................ 2039, 2057

Wang, Dan.... 188, 189, 190, 191, 192

Wang, Dongliang ......................... 1651

Wang, Endi..................................... 1502

Wang, Grace.................................... 537

Wang, Guiqing .................. 1539, 1571

Wang, Guofeng …........................ 1564

Wang, Hai............................ 1069, 1547

Wang, Hanlin L..... 1632, 1649, 1689,

1695

Wang, He.... 318, 331, 337, 468, 1920

Wang, Helen........................ 466, 1739

Wang, Hongbo ................................ 380

Wang, Hongyue ................................. 326

Wang, Hua............................... 726, 750

Wang, Huamin ............. 623, 726, 750

Wang, Huan-You ............................ 1450

Wang, Hui Jun................................ 1070

Wang, Hui Y.................................... 480

Wang, James.......................... 518, 538

Wang, Jian.......................................1112

Wang, Jianmin................................. 138

Wang, Jindong...................................... 337

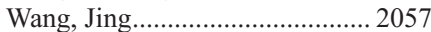

Wang, Jinhua .................................... 185

Wang, Julia Y ............ 829, 833, 1826

Wang, Kai... 146, 241, 271, 827, 1927

Wang, Lai Mun ............................. 1779

Wang, Laura...
Wang, Li........................................ 462 Wang, Li Juan .......... 696, 1547, 2084 Wang, Li-chong................. 2026, 2085 Wang, Liewei .......................... 229, 305 Wang, Lijuan..................... 1069, 1783 Wang, Lisa ........................................... 1963

Wang, Lisha ................. 651, 873, 968 Wang, Lu..... 34, 57, 855, 1071, 1072,

1321,1382

Wang, Michael ................................. 539

Wang, Min............ 487, 503, 515, 537

Wang, Mindy................................ 1542

Wang, Mingsheng .................. 873, 968

Wang, Rui..................................... 1066

Wang, Sa ..... 1377, 1416, 1424, 1473 , $1486,1508,1509$

Wang, Wei .... 1347, 1396, 1509, 1521

Wang, Wei-lien........................... 53, 61

Wang, Weige …………………...... 1403

Wang, Weixin .................................. 1405

Wang, Xi .......................................... 303

Wang, Xiangxue............................. 1583

Wang, Xiaohong I .................. 141, 197

Wang, Xiu Qing .................... 44, 2030

Wang, Xiuhong ................................. 307

Wang, Xiuqing ............................... 281

Wang, Xu ............................. 331, 337

Wang, Xuemei................................ 1023

Wang, Yan .......................... 149, 1254

Wang, Yanhua .................................. 174

Wang, Yihong........... 246, 1324, 1983, 2084

Wang, Yinong.................................. 338

Wang, Yiqin................................... 1245

Wang, Yuxuan ............. 886, 887, 1004

Wang, Zengfeng .......................... 1862

Wang, Zhen ........ 66, 486, 1510, 1511

Wang, Zhen J............................... 1755

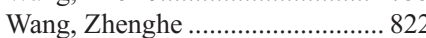

Wang, Zhenglong ......................... 1102

Wang, Zi-Xuan.............................. 2027

Wang, Zixing................................. 1044

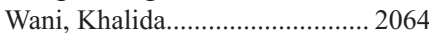

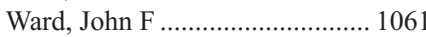

Ward, Kristin ................................... 147

Ward, Michael B .............................. 339

Ward, Pamela M........................... 1092

Ward, Stephen C ........................... 837

Warf, M Bryan ...................................505

Wargo, Jennifer A........................... 535

Warmke, Laura................................... 30

Warmke, Laura M ........................ 2004

Warrick, Joshua I.... 1043, 1127, 1246

Wartchow, Eric P........................ 2043

Wartenberg, Martin ............ 1752, 1780

Wasag, Bartosz............................... 1862

Washington, Mary K ....................... 682

Wasserman, Jason K ... 285, 400, 860, $861,1314,1315,2017$

Watanabe, Mamoru ....................... 1769

Waters, Kevin.................................. 340

Waters, Rebecca ........................... 1687

Watkin, Emmanuel......................... 493

Watkin, William ............................ 1994

Watkins, Jaclyn C............... 1247, 1248

Watkins, Simon ............................. 1560

Watson, Kristalee .......................... 1627

Watson, Linden L ............................. 95

Watson, Lisa C ........... 71, 1409, 2031

Watson, Sarah.................................... 73

Watt, Christopher D

548
Watts, Katherine 957

Weaver, Donald L........................... 116

Webber, Douglas ................................. 12

Weber, Randal S.............................. 1266

Weening, Berber.............................. 900

Wei, Alice

900
747

Wei, Jian-Jun ..................... 1204, 1205

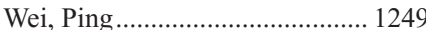

Wei, Shi......... 46, 168, 282, 283, 284 ,

1808

Wei, Wei ...................... 149, 993, 1060

Weidner, Anna-Sophie ........ 659, 817,

2018

Weigelt, Britta ..... 154, 160, 217, 250,

$259,268,939,1185,1250,1798$

1823

Weinberg, Olga K.

1512

Weinbreck, Nicolas .......................... 493

Weinreb, Ilan..................... 1272, 1316

Weinshilboum, Richard M ... 229, 305

Weinstein, John .................... 846, 935

Weinstein, Ronald S.................... 1969

Weisenburger, Dennis D ............ 1329, 1423,1454

Weiser, Martin ....................... 719, 795

Weisman, Paul S ………………... 1257

Weiss, Heidi .................................... 1926

Weiss, Sharon W ………………….... 36

Weiss, Vivian L .................... 469, 818

Weissferdt, Annikka ......... 1906, 1936,

Weixiang, Chen....

... 819

Weizer, Alon Z ………….... 988, 1066

Welch, William R..........................1118

Welker, Kirk M ........................... 1290

Wells, Jason M ................................ 115

Wen, Hannah Y ............. 201, 253, 270

Wen, Huei Chi................................ 250

Wen, K ………………………….... 820

Wen, Kwun Wah ............... 1513, 1688

Wen, Xian-Yu.................................... 753

Wen, Yong Hannah......................... 1798

Wendroth, Scott M ....................... 162

Weng, Weiwei ............. 751, 805, 1245

Wenger, Doris E ................................. 43

Wenham, Robert M........................ 1221, 1240

Wenig, Bruce M ................ 1279, 1294

Wenstrup, Richard J ....................... 505

Wenzel, Sally ............................... 1930

Werlang-Perurena, Ada ...... 105, 1984

Wertheim, Gerald ............... 1514, 1515

West, Dava

West, John ......................... 1792, 2052

West, Robert B ....114, 156, 211, 1973

Westerhoff, Maria .... 759, 1646, 1680

Weston, Jared D ........................... 1293

Westra, William H........... 1267, 1304

1305

Wetzel, Stephanie......................... 1261

Wexler, Deborah ................................. 1096

Wheeler, Ferrin …………………... 155

Wheeler, Michael ............................ 827

White, Christine ..... 1243, 1251, 1317

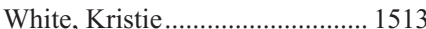

White, Kyle J ..................... 1490, 2019

Whitman, Jeffrey D...................... 1694

Whittington, Elizabeth ........943, 1073

Wieben, Eric D..................... 229, 305

Wieczorek, Rosemary .................... 185

Wieczorek, Tad................................. 458

Wiemels, Joseph............................ 1725

Wiencke, John ................................. 1725

Wiese, Tanya ................................... 477

Wiestner, Adrian........................... 1507

Wik, Elisabeth .................................... 110

Wilcox, Rebecca ……………….......567

Wilcox, Ryan A ............................. 1439

Wilding, Emily ..................... 439, 470

Wilding, Gregory ......................... 1264

Wiles, Austin ..............................30, 471

Wiles, Walter.................................. 1811

Wiley, Elizabeth ................................. 2007

Wilkinson, Edward J .................... 1187

Wilkinson, Nafisa ........................... 1086

Willey, Christopher D ................. 1554

Williams, Erik ..................... 821, 1821

Williams, Kristin E ..................... 1802

Williams, Lindsay ......................... 1271

Williams, Michelle D............ 603, 612

Williams, Nicole ………………..... 158

Williams, Phillip ........................... 1824

Williamson, Sean R..... 853, 873, 917, 933, 955, 956, 964, 968, 985, 1040, 1074, 1075, 1076

Willingham, Field ........................ 1766

Willis, Joseph .................................. 822

Wilson, Parker.............................. 1628

Wilson, Robert S ............................... 954

Wimmer, Daniel .............................. 540

Winblad, Onalisa............................ 265

Windham, Ashley ............................. 95

Winer, Ira .................................... 1089

Winer, Joshua ................................... 773

Winfree, Seth ................................ 2086

Winham, Stacey ........... 132, 133, 204

Winkler, Nicole ............................... 115

Winkler, Thomas .......................... 1357

Winter, Des......................................... 680

Winterhoff, Boris ......................... 2001

Wistuba, Ignacio I... 750, 1457, 1874, 1931, 1937, 2057, 2058

Witt, Benjamin ............................... 1879

Wittman, Bryan ............................. 1032

Witzig, Thomas E........................ 1408

Wludarski, Sheila .............................111

Wobker, Sara E.............................. 1077

Woda, Bruce A ……....................... 152,1523

Wojcik, Eva M .............. 362, 384, 855

Wojcik, John........................ 96, 2087

Wolber, Robert ................................ 281

Wolf, J Stuart..................... 1039, 1066

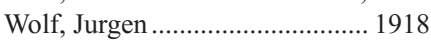

Wolfe, Gregory..................................2 29

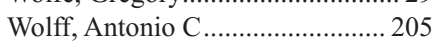

Wolff, Robert A.................... 726, 750

Wolfgang, Christopher L................ 812

Won, Helen..................................... 1910

Won, Jennifer ............ 281, 2020, 2021

Won, Nam Hee............................... 707

Wong, Jerry T................................. 1329

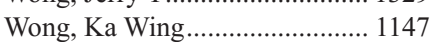

Wong, Kristine S ...................................... 613

Wong, Richard ............................... 1320

Wong, Richard WC ...................... 1147

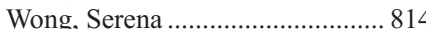

Wong, Waihay ........................................ 1516

Wood, Adam J ................................ 1951

Wood, Brent L.................... 1394, 1531

Wood, Laura.................................... 769

Wood, Stephanie M................ 678, 823

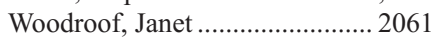

Woods, Robbie ............................. 1317 
Woods, Tina .................................... 1299

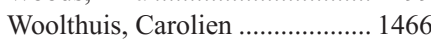

Worral, Amber................................. 193

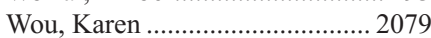

Wouters, Jasper ............................... 492

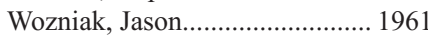

Wozniak, Laura ............................. 1694

Wrensch, Margaret...................... 1725

Wright, Jesse P ................................ 469

Wright, Nikita …………………......... 304

Wronowska, Weronika ................... 935

Wu, Angela................... 108, 183, 987

Wu, Annie ... 1440, 1517, 1518, 1689 ,

Wu, Cecilia. 1896

Wu, Chin-Lee................................ 1306

Wu, David ......................... 1394, 1531

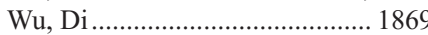

Wu, Frances ................................ 1731

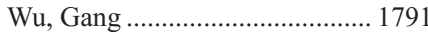

Wu, Howard ..................................... 404

$\mathrm{Wu}, \mathrm{JIn}$...................................... 40, 41

Wu, Jonn ...................................... 1282

Wu, Richard L.......... 824, 1825, 1848

Wu, Tao .............................. 472, 1256

Wu, Tongtong ........................................ 620

Wu, Tsung-Teh ........ 714, 1643, 1644,

1645

Wu, Xuemei ............... 229, 305, 2093

Wu, Xun ........................................... 509

$\mathrm{Wu}$, Yongping ………………......... 212

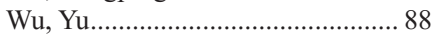

Wu, Yun................ 106, 147, 150, 307

Wunder, Jay S .................................. 54

Wykes, James ................................ 1292

Wyse, Aaron J .............................. 1404

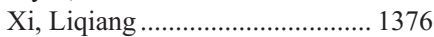

Xia, Daniel ........................ 1733, 1938

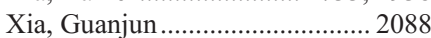

Xia, Jun ............................... 304, 826

Xia, Michelle...................................... 825

Xian, Wa........................................ 1252

Xiao, Guang-Qian ..... 857, 858, 1051,

1216,1924

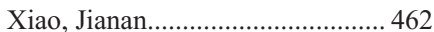

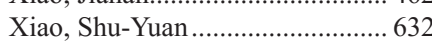

Xiao, Wenbin...................... 1519, 1520

Xiao, Yaoxing................................ 311

Xie, Hao ……………….... 1662, 1663

Xie, Hong ............................................ 514

Xie, Wei............................................ 1521

Xie, Xian-Jin .................................... 914

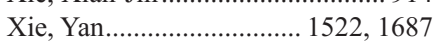

Xie, Yi .................................................. 1502

Xin, Wei .................................. 661, 840

Xing, Wei .......................... 1523, 1524

Xinyan, $\mathrm{Lu}$.................................... 1341

Xiong, Changming ..............................326

Xiong, Jianghong .............................. 312

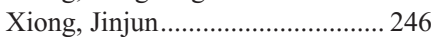

Xiong, Yin ...................................... 895

Xiong, Yiqin................................... 1523

Xu-Monett, Zijun Y.................... 1522

Xu, Bin.......... 614, 1318, 1319, 1320 ,

1321

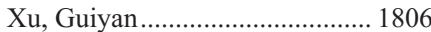

Xu, Guoping........................ 1873, 1874

Xu, Haiying .................................... 293

Xu, Haodong ........... 1122, 1649, 1924

$\mathrm{Xu}$, Huihong........................... 376, 386

$\mathrm{Xu}$, Jessica.

1280
Xu, Jie .................................. 94, 1525

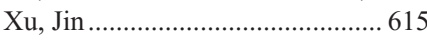

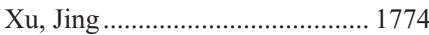

Xu, Mi-die................................................... 805

Xu, Midie ........................... 751, 1245

Xu, Mina L................................... 1453

$\mathrm{Xu}$, Qing............................................. 312

Xu, Ruliang ...................... 1629, 1654

Xu, Shuo …………………….......... 1939

Xu, Xinjie......................................... 1339

Xu, Xiongfei .................................. 1782

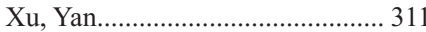

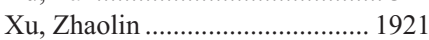

Xue, Yue.......... 772, 826, 1738, 1772,

1784

Yabe, Mariko.................................... 1526

Yaffe, Martin J ............................ 2025

Yahagi, Kazuyuki............................ 322

Yakirevich, Evgeny ............. 758, 809, $827,974,1069,1123,1313$ $1324,1729,1783$

Yamada, Shin ...................................... 1063

Yamada, Yuichi .................................. 70

Yamaguchi, Rin............................... 227

Yamamoto, Hidetaka.................. 70, 97

Yamamoto, Tomohiko .................... 735

Yamamoto, Yusuke........................ 1252

Yamanaka, Kazuteru ..................... 1888

Yamanishi, Douglas T ................. 2089

Yamano, Yasuhiko......................... 1867

Yamasaki, Takashi.............. 177, 1656

Yamashita, Hiroharu ........................ 619

Yamauchi, Shuta ......................... 1888

Yamazaki, Sanae ………………….... 72

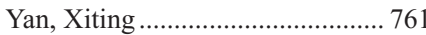

Yan, Zhantao .................................. 212

Yanagawa, Naoki ........................... 1940

Yanagihara, Eugene........................ 2068

Yanagisawa, Akio................................. 72

Yanaihara, Nozomu....................... 1155

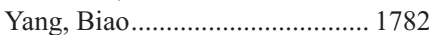

Yang, Bin...................................... 1256

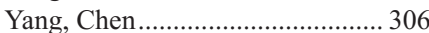

Yang, Ching-Fen ........................... 134

Yang, Chongqing........................... 2090

Yang, David T ................................ 1451

Yang, Dongfang .......... 246, 678, 696, $823,1069,1547$

Yang, Eric........................ 1180, 1247

Yang, Fei ………………………...... 307

Yang, Gloria ................................... 1370

Yang, Guang-Yu........ 577, 666, 1532, $1635,1636,1652,1758,1807$

Yang, Haichun ............................... 1596

Yang, Huaitao................................... 473

Yang, Hui-Min ................................ 694

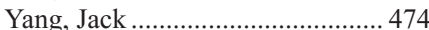

Yang, Jing............................ 200, 238

Yang, Jung Wook ........................... 828

Yang, Miso ……………………....... 2048

Yang, Ping ................................... 1855

Yang, Qi ................... 857, 858, 1051

Yang, Qian........... 317, 637, 829, 833,

1826

Yang, Shi ............................... 502, 516

Yang, Wan Rou ............................ 1429

Yang, Wentao ..............311, 1101, 1112

Yang, Xiaojing .......................... 1856

Yang, Ximing ........ 69, 382, 419, 855,

1014,1072

Yang, Xiu

$475,477,1322$
Yang, Youling.............................. 1144

Yang, Zhaohai ............ 717, 830, 1690

Yang, Zhongbo ................................ 476

Yano, Hirohisa...................... 227, 1223

Yantiss, Rhonda K....... 659, 660, 693,

719,817

Yao, Hui ........................................... 444

Yao, James C............................... 1873

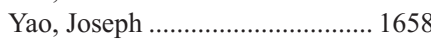

Yao, Keluo........................................ 1941

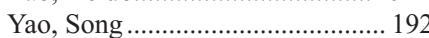

Yap, Kai Lee.................................... 1504

Yaprak Bayrak, Busra .................. 1184

Yaqub, Abid..................................... 473

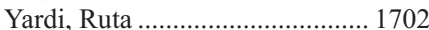

Yaseen, Alae .................................... 477

Yasir, Saba....................................... 745

Yaskiv, Oksana ........... 977, 979, 2015

Yasuda, Kaori ................................ 1223

Yau, Christina........................ 121, 122

Yau, Jonathan ............................... 2058

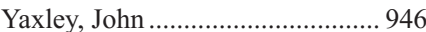

Yazdani, Samaneh..............................583

Yaziji, Hadi ...................................... 228

Ye, Bo.............................. 1122, 1924

Ye, Fei ………………….................... 541

Ye, Huihui ........................ 1052, 1078

Ye, Xiaobu..................................... 1703

Yee, Eric........................................ 1739

Yeh, Matthew .......... 776, 1647, 1657,

1671

Yekezare, Somaye .............. 478, 2022

Yemelyanova, Anna .........1125, 1172,

1253

Yenamandra, Ashwini .................... 155

Yeong, Joe............................ 176, 177

Yergiyev, Oleksandr.......................... 52

Yerian, Lisa ................................ 1632

Yi, Chunhui ........................................ 541

Yi, Eunhee S.......... 1849, 1855, 1881,

Yi, Kijong...................................... 1691

Yilmaz, Funda ............................. 1692

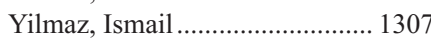

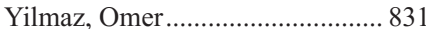

Yilmaz, Osman....................831, 1630

Yin, C Cameron .... 1374, 1388, 1389, $1390,1391,1456,1492$

Yin, Cheng C............................... 1525

Yin, Fufen ……............................ 482

Yin, Hong............................................ 1942

Ying, Zhitao ……………………... 1481

Yip, Stephen................44, 2020, 2021

Yoder, Sean ................................... 122

Yokota, Mitsuru..................... 739, 832

Yonescu, Raluca............................... 261

Yoo, Su Hyun .....................1162, 1163

Yoon, Esther....................................... 308

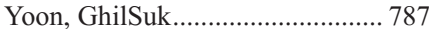

Yoon, J-Y ………………..... 833, 834

Yoon, Jiyoon ................................. 1041

Yordanka, Reyna ............... 1663, 1664

York, Eric B ................................. 1770

Yorozu, Takashi........................... 1037

Yoshida, Akihiko

.... 97

Yoshida, Misa.............................. 1845

Yoshimoto, Toyoki .............................. 98

Yoshimura, Masayo......................... 1866

Yoshino, Yasushi .......................... 1063

Yoshizawa, Akihiko ......... 1864, 1913,

1920
You, Jia..

You, M James................................ 1525

You, Xiaoming .......... 577, 666, 1532, $1635,1636,1652$

Younes, Anas.......... 1382, 1481, 1803

Young Pierce, Jennifer ................. 1208

Young, Keith ................................. 2051

Young, Ken H........ 1368, 1457, 1522,

1526

Young, Neal...................... 1356, 1357

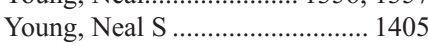

Young, Robert H ……………….. 1133

Youngson, Bruce J ........................ 1824

Yousaf, Hira ....................................... 20

Yousef, George M..... 896, 981, 1033 ,

1034,1057

Yousefi, Elham ............. 19, 704, 1569

Yousefi, Kasra .................................. 906

Yousem, Samuel A ........... 1557, 1890, 1930

Yoxtheimer, Lorene........................ 417

Yu, Bing ............................................ 1293

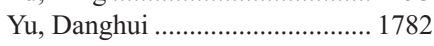

Yu, Elliot ....................................... 1280

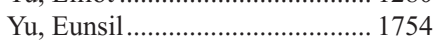

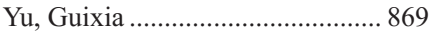

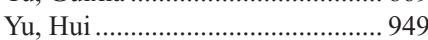

Yu, Jiangzhou ................................. 643

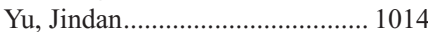

Yu, Jing ........................................... 479

Yu, Shan-Chi ............................... 1527

Yuan, Ji........ 1351, 1352, 1365, 1366,

1450,1655

Yuan, Lisi ........................................... 835

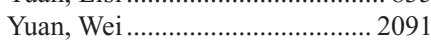

Yuan, Xin .................................... 1078

Yucesoy, Izzet ................................. 1184

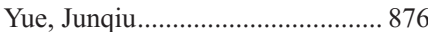

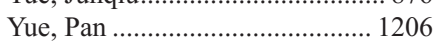

Yue, Yong ............................ 109, 309

Yuen, Nancy WF ........................... 1147

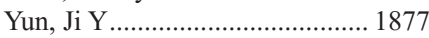

Yun, Ji Yun ....................................... 522

Yusa, Toshikazu .......................... 1869

Yusifli, Zerife ........................ 392, 636

Yusuf, Muhammed A ...................... 737

Yusuf, Yasmin .............................. 1852

Zabor, Emily C............................... 847

Zaccarini, Daniel............................... 99

Zafar, Nadeem................................ 743

Zafeiriou, Zafeiris ................................ 1001

Zafon, Carles................................. 2073

Zahavi, David J .............................. 934, 1054

Zahurak, Marianna ........................ 812

Zai, Ertao.................................... 691

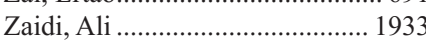

Zaidi, Syed ........................................ 380

Zaino, Richard...................... 1219, 1246

Zaki, Sherif R............................. 1538

Zaloudek, Charles ..............1165, 1175 ,

1189,1233

Zamboni, Giuseppe .......... 1738, 1772

Zanatta, Lucia .................................. 84

Zanconati, Fabrizio ..........................111

Zander, Dani S ................................. 1968

Zarbo, Richard J............... 1961, 2092

Zarei, Shabnam ................. 1254, 2093

Zarrabi, Nikou....................................... 1748

Zavodszky, Maria.............................. 161

Zeck, Jay .......................................... 346

Zeh, Herbert J

1775,1776 
Zehir, Ahmet ...... 64, 270, 1801, 1814 Zehnder, Jim..................... 1758, 1807

Zeitouni, Joseph ........................... 1550

Zelenetz, Andrew D .......... 1382, 1481

Zelonis, Michele K........................ 1942

Zemek, Allison J .......................... 1781

Zen, Yoh.......................... 1656, 1746

Zenali, Maryam............................. 1963

Zeng, Chun...................................... 1623

Zeng, Gang.................................. 1616

Zeng, Jennifer ..................... 616, 1255

Zeng, Yu ..................................... 1363

Zeng, Zhengyu .................................. 484

Zera, Richard................................... 182

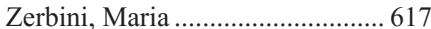

Zhan, Yang ..................................... 185

Zhang, Bingqing .... 2026, 2051, 2085

Zhang, Cathy...................................5 578

Zhang, Chen ................................... 1943

Zhang, Da...................................... 2061

Zhang, David............... 454, 541, 1284

Zhang, Gloria ..................... 310, 1256

Zhang, Guojuan............................ 1529

Zhang, Hailing .................. 1400, 1528

Zhang, Haitao................................. 185

Zhang, Hao................................... 462

Zhang, Hongfeng ................ 781, 1852

Zhang, Jiexin............ 642, 1104, 1788

Zhang, Jing.................................. 1782

Zhang, Jinghui............................. 1791

Zhang, Jun............................936, 937

Zhang, Lei......... 33, 47, 58, 480, 1316

Zhang, Li.............................. 891, 935

Zhang, Lihong................................ 462

Zhang, Ling...................... 1444, 1501

Zhang, Lingxin.................... 306, 1323

Zhang, Linsheng ........................ 1578

Zhang, Lizhi............... 715, 803, 1756

Zhang, Miao....... 891, 912, 951, 1022

Zhang, Ming................................ 1827

Zhang, Mingjuan L ..................... 1734

Zhang, Pan ............................. 347, 693

Zhang, Ping L

1605

Zhang, Pingchuan

100

Zhang, Qing

1204,1205

Zhang, Qiongyan......... 751, 805, 1245

Zhang, Ranran............ 615, 618, 1257

Zhang, Rongzhen ........................ 1428

Zhang, Rui.................................... 1524

Zhang, Shanxiang …................... 1559

Zhang, Shaobo ...................... 873, 968

Zhang, Shengle .............. 99, 432, 434

Zhang, Shizhen .............................. 891

Zhang, Songlin............. 141, 197, 289

Zhang, Tianzhu .............................. 916

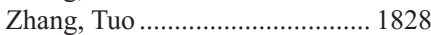

Zhang, Weiwei ............................. 1101

Zhang, Xiao................................... 309

Zhang, Xiaofei ..................... 836, 837

Zhang, Xiaohui .................. 749, 1528

Zhang, Xinmin ............................ 1380

Zhang, Xuchen..... 341, 761, 814, 815

Zhang, Xuefeng................................ 688

Zhang, Xun ....................................... 876

Zhang, Yang .................... 31, 32, 1693

Zhang, Yanhong ............................. 121

Zhang, Yanmin ................................... 793

Zhang, Yanming ........................... 1334

Zhang, Yaxia .................................... 481

Zhang, Yonghua ................ 2054, 2084

Zhang, Yuekai
Zhang, Zesong................................. 454

Zhang, Zhen .................................... 1875

Zhang, Zhenwei ........................... 1078

Zhang, Zhiqing................... 2054, 2084

Zhang, Ziying............................... 1085

Zhao, Chaohui L .............. 1324, 1783

Zhao, Chengquan ........ 457, 462, 472,

$482,483,484$

Zhao, Chenyan ............................... 1782

Zhao, Chong............................... 1422

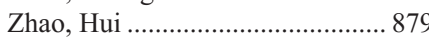

Zhao, Jing-min ............................ 1542

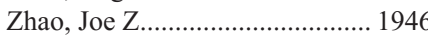

Zhao, Kevin Y ............................... 1829

Zhao, Lei ............................ 710, 1632

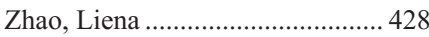

Zhao, Ming............................................... 876

Zhao, Qing ....................................... 804

Zhao, Song Q ................... 2054, 2084

Zhao, Tianhao ………………...... 1579

Zhao, Weiqiang ..... 1016, 1529, 1829 ,

1941

Zhao, Xiaomu …………………....... 1616

Zhelnin, Kristen E......................... 1784

Zheng, BaoWen............. 472, 482, 484

Zheng, Gang....................... 984, 1916

Zheng, Guangjuan........................... 312

Zheng, Mei ........................ 1352, 1530

Zheng, Qizhi ................................... 931

Zheng, Wenxin ...............................1110

Zheng, Xiaoyong........................... 1080

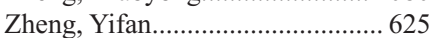

Zheng, Zhongxi........................... 1573

Zhong, Minghao............. 69, 781, 854, $1159,1160,1851,1852$

Zhong, Minhao................................ 308

Zhou, Delu ....................................... 1584

Zhou, Haijun ..................................... 371

Zhou, Haijun (Steve)........................ 485

Zhou, Jiehao ...................... 1559, 1680

Zhou, Lan................................................ 661

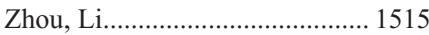

Zhou, Lixin ................................... 876

Zhou, Ming ......... 855, 881, 905, 933, 949, 966, 977, 978, 979, 983, $1042,1062,1074,1081$

Zhou, Shuling.................................. 311

Zhou, Wendi............ 1632, 1694, 1695

Zhou, Xianrong ........... 462, 482, 1245

Zhou, Xiaoyan...................... 651, 1403

Zhou, Yaolin......................... 486, 2023

Zhou, Yi ......... 838, 839, 1352, 1394,

1450, 1521, 1531, 1576, 1790,

1975,1991

Zhou, Yu.................................................... 1583

Zhou, Zhongren...................... 620, 643

Zhu, Andrew ....................................... 1630

Zhu, Benjamin.................................. 775

Zhu, Bin .......................... 1738, 1772

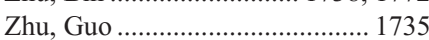

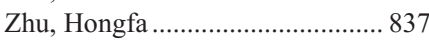

Zhu, Hongguang ........................... 1885

Zhu, Jun........................................ 1551

Zhu, Menglei.................................. 840

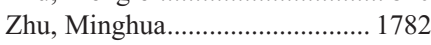

Zhu, Shaobo …………………........... 1942

Zhu, Shirley X............................... 211

Zhu, Ya-Zhen .................................. 312

Zhuge, Jian.................................. 1539

Zia, Hamid ... 1345, 1355, 1817, 1944

Ziemnik, Suzanne M................... 1432
Zimmer, Lisa ................................. 1721

Zimmermann, Michael................. 1363

Ziober, Amy …............................. 1298

Zippin, Jonathan H........................ 1055

Zirpoli, Gary .................................. 193

Ziskin, Jennifer ........................... 1973

Zlobec, Inti.......... 662, 663, 664, 670,

1482

Zoino, Roberto .................... 983, 1081

Zoppo, Franco …………………..... 332

Zorn, Kristin................................ 2065

Zoroquiain, Pablo............. 1736, 1737

Zreik, Riyam T....... 35, 95, 566, 1984

Zsengeller, Zsuzsanna K............ 1137

Zukerberg, Lawrence ........... 667, 796,

797, 821, 1337, 1387, 1546, 1653

Zuna, Rosemary ........................... 1946

Zuo, Chunlai ................................. 1082

Zuo, Zhuang ............ 149, 1374, 1388, $1389,1390,1391,1422$

Zuppan, Craig ............................. 1606 zur Hausen, Axel ......................... 1387

Zweidler-McKay, Patrick A ........ 1424

Zynger, Debra L .......... 159, 172, 181,

$263,600,1016$ 